\title{
Development and Characterization of Potent Cyclic Acyldepsipeptide Analogues with Increased Antimicrobial Activity
}

Jordan D. Goodreid, John Janetzko, John P. Santa Maria Jr., Keith S. Wong, Elisa Leung, Bryan T. Eger, Steve Bryson, Emil F. Pai, Scott D. Gray-Owen, Suzanne Walker, Walid A. Houry, and Robert A. Batey*

Davenport Research Laboratories, Department of Chemistry, University of Toronto, 80 St. George Street, Toronto, ON M5S 3H6, Canada

\author{
Fax: (1+) 416-978-5059 \\ *Email: rbatey@chem.utoronto.ca \\ SUPPORTING INFORMATION
}

Table of Contents

Pages

1 Supplemental Information

Figures $\mathrm{S} 1$

Figures S2

Table S1

$2 \quad{ }^{1} \mathrm{H}$ and ${ }^{13} \mathrm{C}$ NMR Spectra for Numbered Compounds

8, 9, 12a, 12b, 13a-c, 14a-f, 15a-f, 16a-f, 17a-f, 19a-f, 20a-f, 21a-e, and 22-30

3 HPLC Chromatograms for Numbered Compounds

$4,7,8,9,22-28$, and $30-38$

S60-S70

$4 \quad$ References

S71-S72 


\section{Supplemental Information}
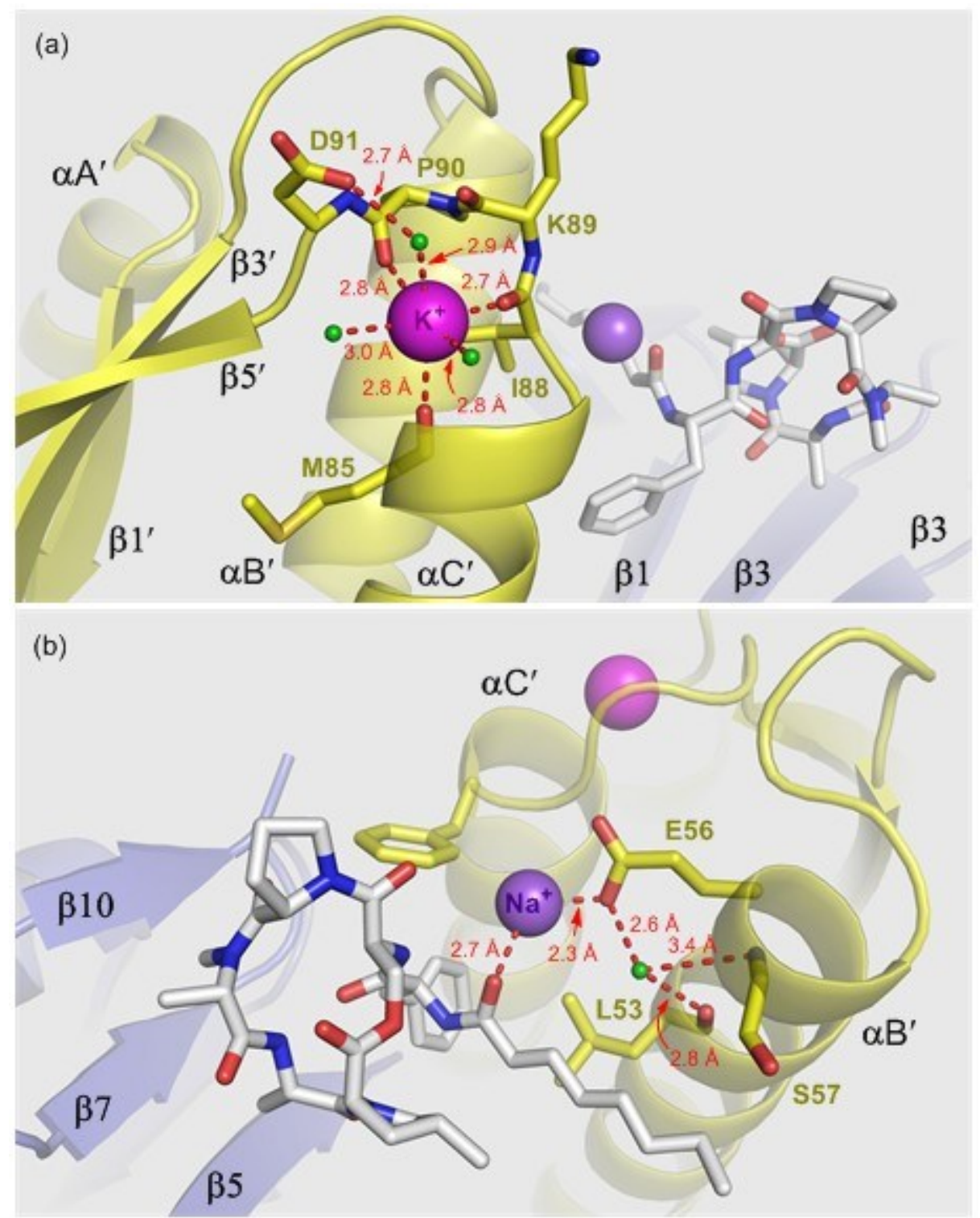

Figure S1. Close-up view of cations bound close to the Factor D (4) binding site (here subunit c). (a) The magenta sphere represents a potassium ion that is ligated to the backbone carbonyls of Met85, Ile88, and Pro90 as well as three tightly bound water molecules (green spheres). (b) The purple sphere indicates the position of a sodium ion. It is bound to the amide oxygen of Factor D's octadienoic acid side chain and to one of the carboxylate oxygens of Glu56, which in turn is bridged to the carbonyl oxygen of Leu53 by a water molecule. While this water molecule and the potassium ions, the latter with varying numbers of water ligands, are found in all 28 subunits the sodium ions could be identified with confidence in only three subunits, c, h, and k. For simplicity, the van der Waals radii of the cations have been scaled down roughly $50 \%$ from their actual size. 


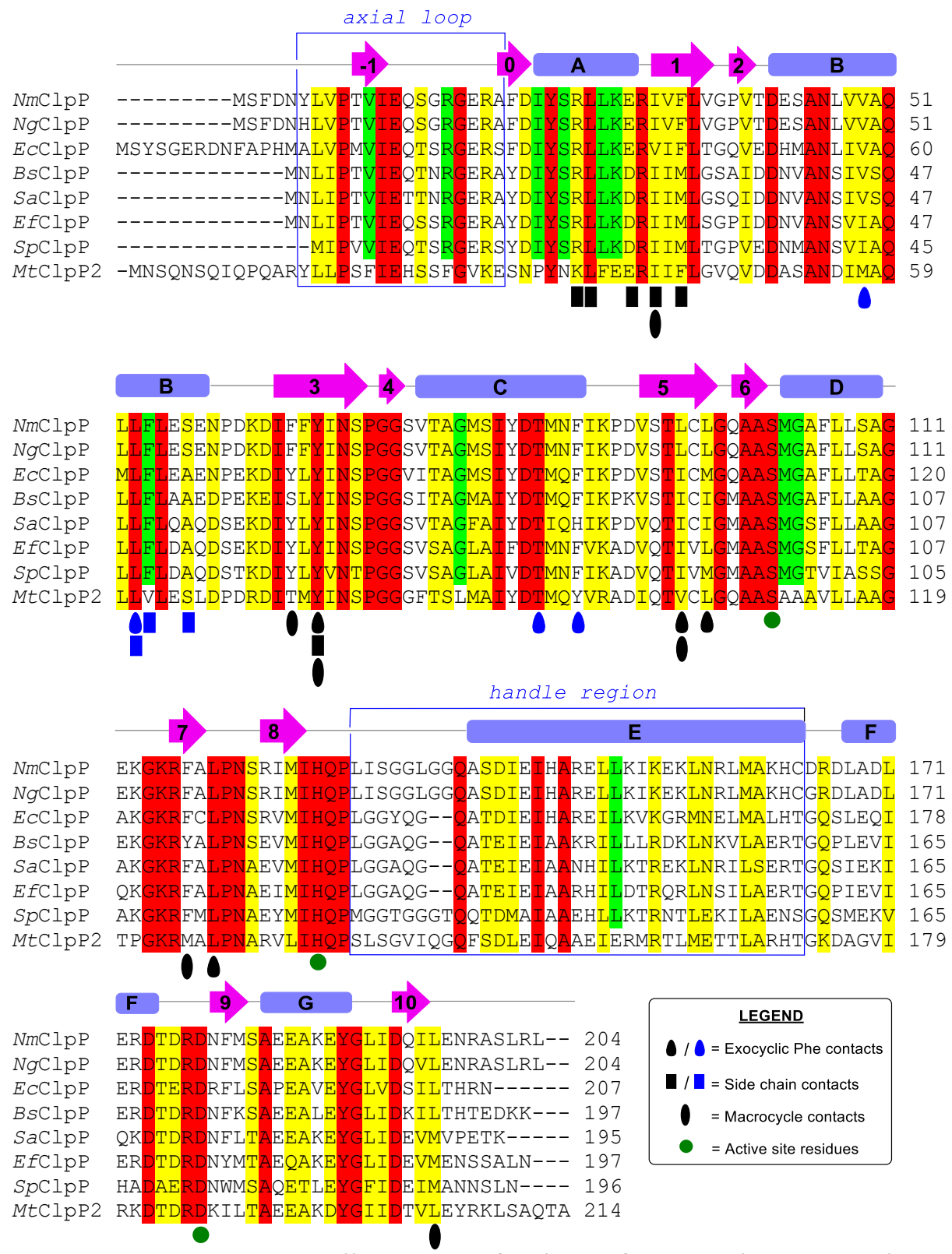

Figure S2. Sequence alignment of ClpPs from various organisms: N. meningitidis H44/76 (NmClpP) [E6MZ69], N. gonorrhoeae ATCC 700825/FA 1090 (NgClpP) [Q5F914], E. coli K12 (EcClpP) [P0A6G7], B. subtilis 168 (BsClpP) [P80244], S. aureus subsp. aureus NCTC 8325 (SaClpP) [Q2G036], E. faecalis ATCC 700802/V583 (EfClpP) [Q837R0], S. pneumoniae strain ATCC BAA-255 / R6 (SpClpP) [P63788], M. tuberculosis ClpP2 (MtClpP2) [P9WPC3]. The alignment was generated using Clustal Omega $^{1}$ and the ClpP sequences were obtained from the UniProt database (UniProt ID codes are shown in square brackets). Secondary structure elements for $N m \mathrm{ClpP}$ are shown above its sequence ( $\alpha$-helicies, light blue; $\beta$-sheets, magenta). Highlighting indicates residues which are identical in all sequences (red), identical in seven of the ClpP sequences (green), or are highly conserved in all sequences (yellow). The interactions of different portions of the ADEP with ClpP, which are based on existing Xray structures, are indicated by symbols that appear below the interacting residue and are defined in the accompanying legend (ClpP monomer 1, black; monomer 2, blue). 
Table S1. Bacterial strains.

\begin{tabular}{|c|c|c|}
\hline Strain & $\begin{array}{l}\text { Relevant genotype/identity and antibiotic } \\
\text { sensitivity }\end{array}$ & Ref. \\
\hline \multicolumn{3}{|l|}{ Gram Positive } \\
\hline $1784 \mathrm{~A}$ & $\begin{array}{l}\text { Staphylococcus aureus; methicillin- } \\
\text { resistant clinical isolate }\end{array}$ & 2 \\
\hline Newman & $\begin{array}{l}\text { Staphylococcus aureus subsp. aureus } \\
\text { Newman; methicillin-sensitive human } \\
\text { clinical isolate }\end{array}$ & 3 \\
\hline ATCC 29213 & $\begin{array}{l}\text { Staphylococcus aureus subsp. aureus } \\
\text { Rosenbach; oxacillin-sensitive wound } \\
\text { isolate }\end{array}$ & 4 \\
\hline V583 & $\begin{array}{l}\text { Enterococcus faecalis; vancomycin- } \\
\text { resistant blood isolate }\end{array}$ & 5 \\
\hline PY79 & $\begin{array}{l}\text { Prototrophic derivative of Bacillus subtilis } \\
\text { subsp. subtilis } 168\end{array}$ & 6 \\
\hline D39 & Streptococcus pneumoniae D39 & 7 \\
\hline L. innocua & Wild-type Listeria innocua & 8 \\
\hline \multicolumn{3}{|l|}{ Gram Negative } \\
\hline E. coli & Wild-type Escherichia coli MC4100 & 9 \\
\hline LptD4213 & Escherichia coli lptD-4213 & 10 \\
\hline PA01 & Pseudomonas aeruginosa PA01 & 11 \\
\hline N.279 & $\begin{array}{l}\text { Neisseria gonorrhoeae N.279 (S, } \\
\text { opaC::cat })^{a}\end{array}$ & 12 \\
\hline $\mathrm{H} 44 / 76$ & Neisseria meningitidis $\mathrm{H} 44 / 76$ & 13 \\
\hline $\mathrm{H} 44 / 76 \Delta c l p P$ & $\begin{array}{l}\text { Neisseria meningitidis } \mathrm{H} 44 / 76 \text { isogenic } \\
\text { clpP-deficient mutant }\end{array}$ & 14 \\
\hline
\end{tabular}

${ }^{a}$ This strain is a marked allelic replacement of opaC and carries a gene encoding a chloramphenicol acetyltransferase (cat) thereby rendering the strain chloramphenicol-resistant. 


\section{$2 \quad{ }^{1} \mathrm{H}$ and ${ }^{13} \mathrm{C}$ NMR Spectra for Numbered Compounds}

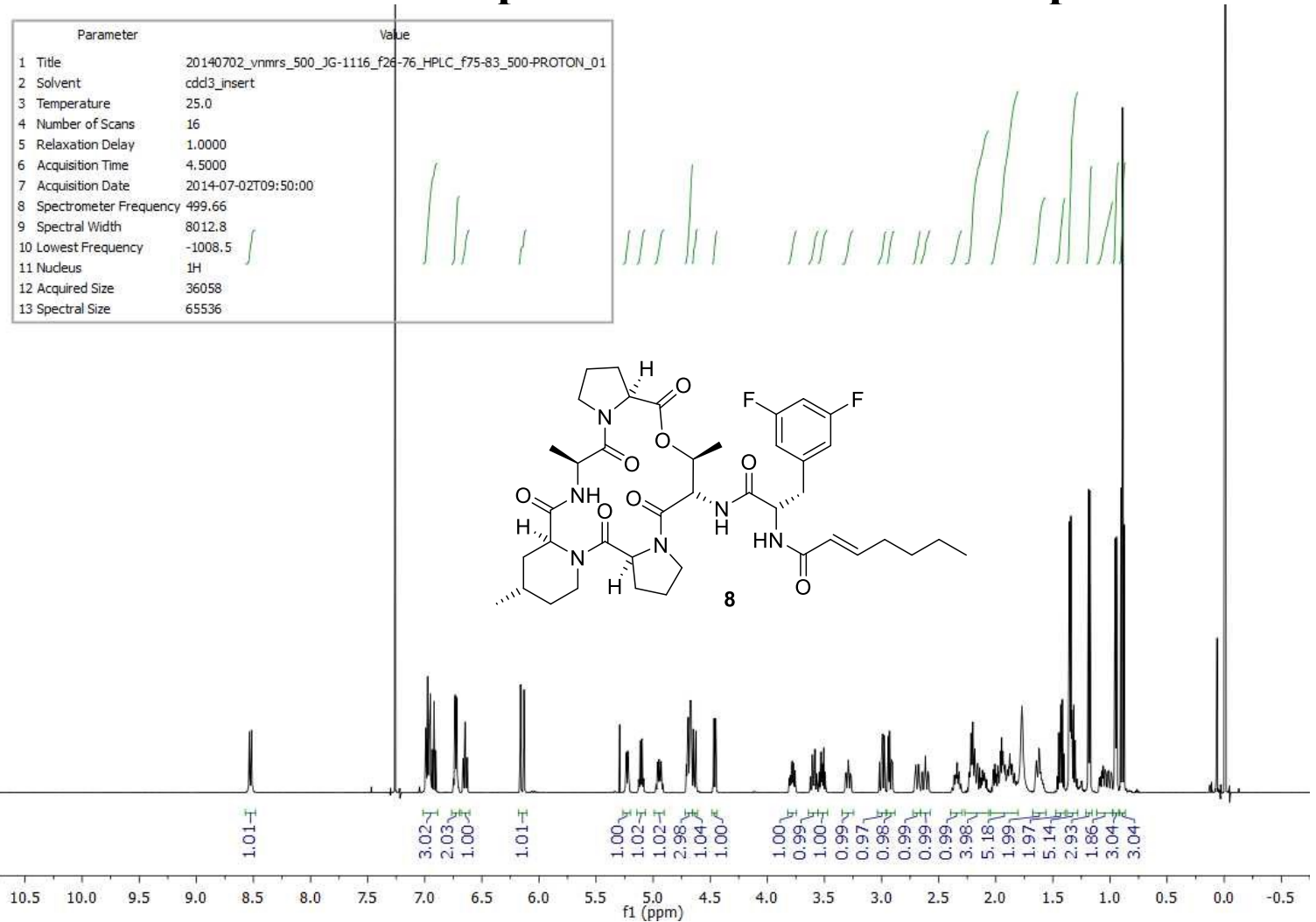

\begin{tabular}{|lll|}
\hline \multicolumn{2}{|c|}{ Parameter } & \multicolumn{1}{c|}{ Value } \\
1 & Tite & 20140702 _ynmrs_500_JG-1116_f26-76_HPLC_f75-83_500-CARBON_01 \\
2 & Solvent & cdd13_insert \\
3 & Temperature & 25.0 \\
4 & Number of Scans & 2000 \\
5 & Relaxation Delay & 0.5000 \\
6 & Acquisition Time & 2.0000 \\
7 & Acquisition Date & $2014-07-02721: 58: 23$ \\
8 & Spectrometer Frequency 125.65 \\
9 & Spectral Width & 30487.8 \\
10 & Lowest Frequency & -2031.3 \\
11 & Nucleus & $13 \mathrm{C}$ \\
12 & Acquired Size & 60976 \\
13 Spectral Size & 131072 \\
\hline
\end{tabular}

$\begin{array}{llllllllllll}220 & 210 & 200 & 190 & 180 & 170 & 160 & 150 & 140 & 130 & 120 & 110 \\ \mathrm{f} 1(\mathrm{ppm}) & 100\end{array}$ 


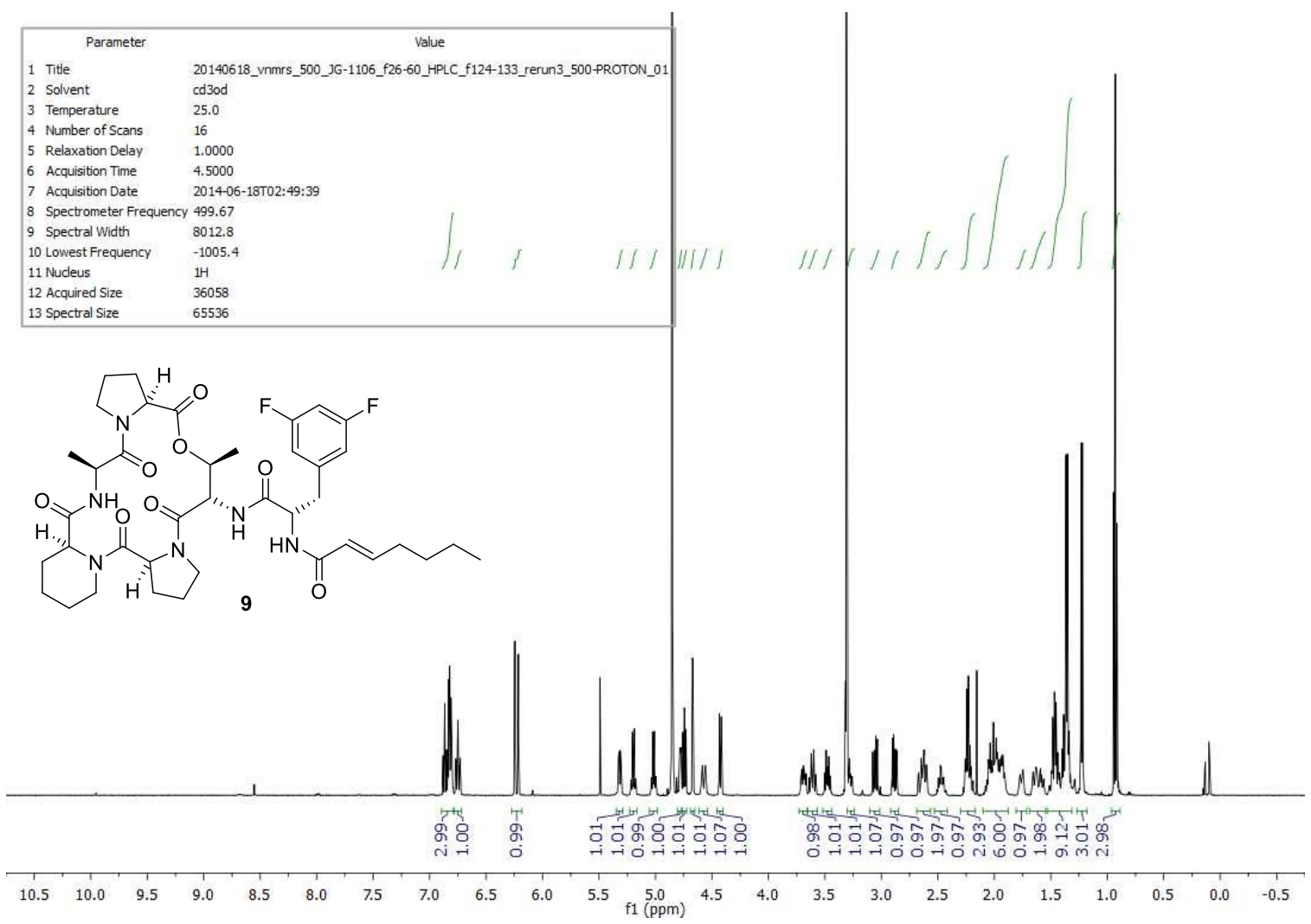

\begin{tabular}{|c|c|}
\hline Parameter & Value \\
\hline 1 Title & 20140618_ynmrs_500_JG-1106_f26-60_HPLC_f124-133_rerun3_500-CARBON_01 \\
\hline 2 Solvent & cd3od \\
\hline 3 Temperature & 25.0 \\
\hline 4 Number of Scans & 2000 \\
\hline 5 Relaxation Delay & 1.0000 \\
\hline 6 Acquisition Time & 2,0000 \\
\hline 7 Acquisition Date & 2014-06-18T02:51:33 \\
\hline 8 Spectrometer Frequency & 125.65 \\
\hline 9 Spectral Width & 30487.8 \\
\hline 10 Lowest Frequency & -1853.3 \\
\hline 11 Nucleus & $13 \mathrm{C}$ \\
\hline 12 Acquired Size & 60976 \\
\hline 13 Spectral Size & 131072 \\
\hline
\end{tabular}
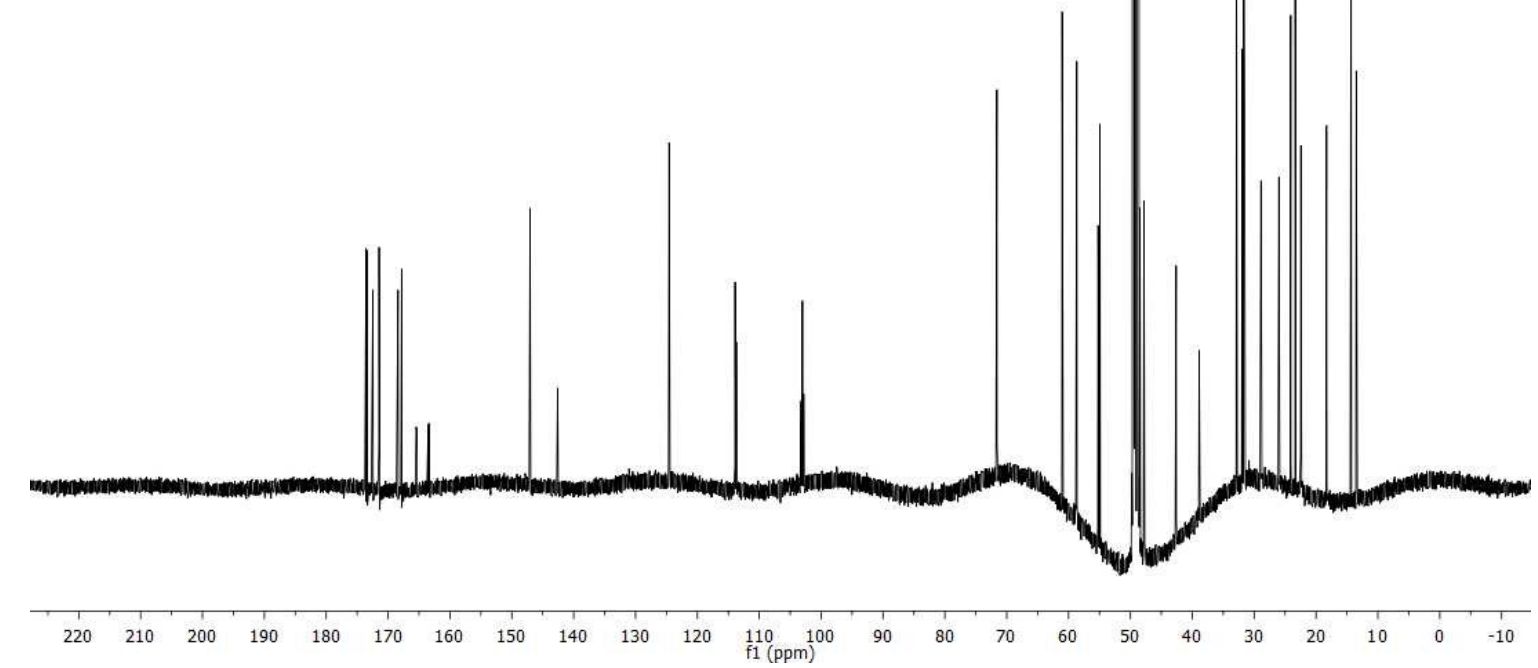


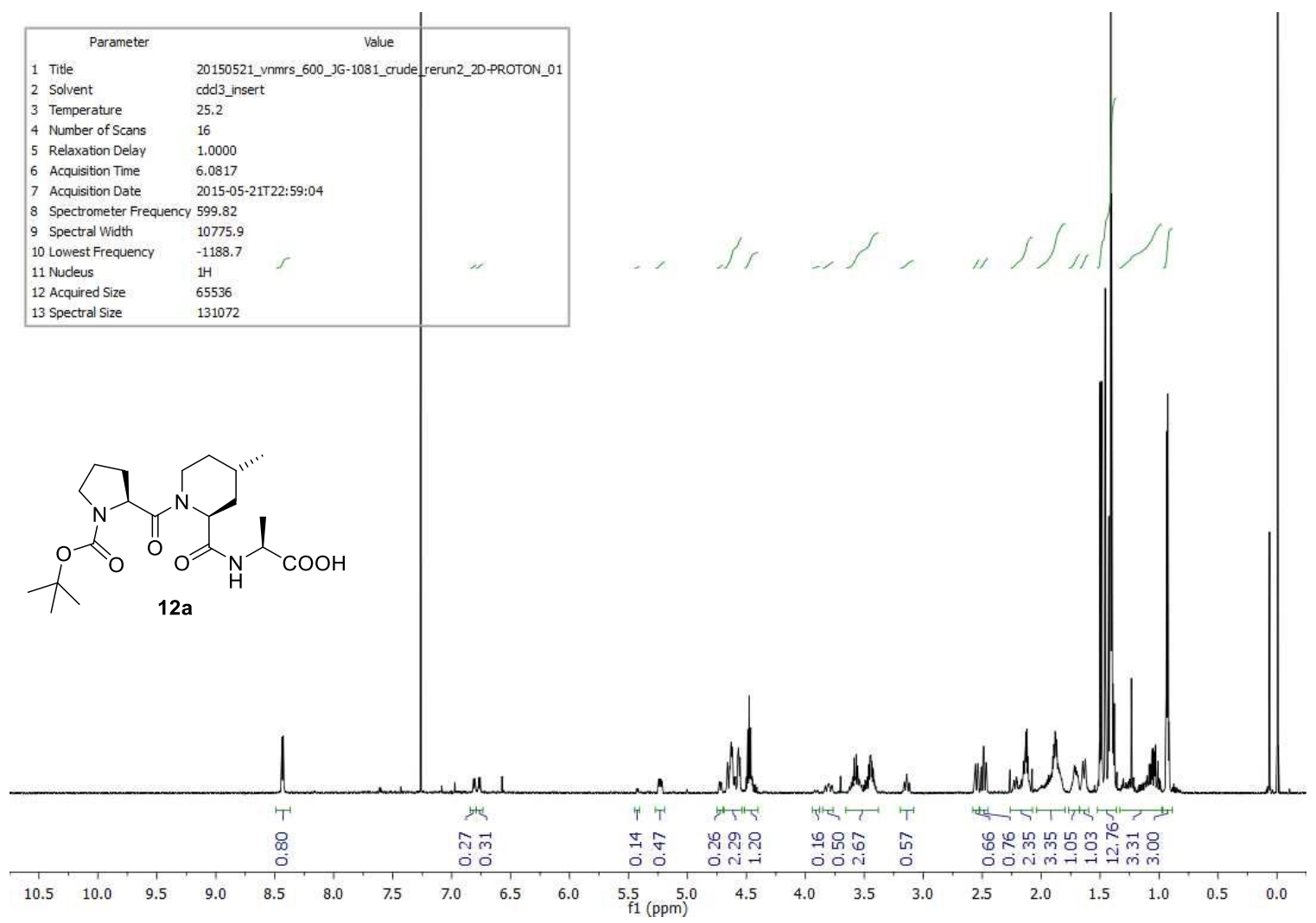

\begin{tabular}{|lll|}
\hline \multicolumn{1}{|c|}{ Parameter } & \multicolumn{1}{c|}{ Value } \\
1 & Title & 20150522 ynmrs_500_JG-1081_crude_13C_rerun-CARBON_01 \\
2 & Solvent & cdd3_insert \\
3 & Temperature & 25.0 \\
4 & Number of Scans & 750 \\
5 & Relaxation Delay & 0.2000 \\
6 & Acquisition Time & 2,0000 \\
7 & Acquisition Date & $2015-05-22 T 20: 21: 43$ \\
8 & Spectrometer Frequency 125.65 \\
9 & Spectral Width & 30487.8 \\
10 Lowest Frequency & -2031.3 \\
11 Nucleus & 13 C \\
12 Acquired Size & 60976 \\
13 & Spectral Size & 131072 \\
\hline
\end{tabular}




\begin{tabular}{|c|c|}
\hline Parameter & Value \\
\hline 1 Title & 20150521_vnmrs_400_JG-402_crude__erun_2D-PROTON_01 \\
\hline 2 Solvent & cdd3_insert \\
\hline 3 Temperature & 25.0 \\
\hline 4 Number of Scans & 16 \\
\hline 5 Relaxation Delay & 1.0000 \\
\hline 6 Acquisition Time & 4.5613 \\
\hline 7 Acquisition Date & 2015-05-21T22:35:00 \\
\hline 8 Spectrometer Frequency & 399.74 \\
\hline 9 Spectral Width & 7183.9 \\
\hline 10 Lowest Frequency & -786.6 \\
\hline 11 Nudeus & - \\
\hline 12 Acquired Size & 32768 \\
\hline 13 Spectral Size & 65536 \\
\hline
\end{tabular}

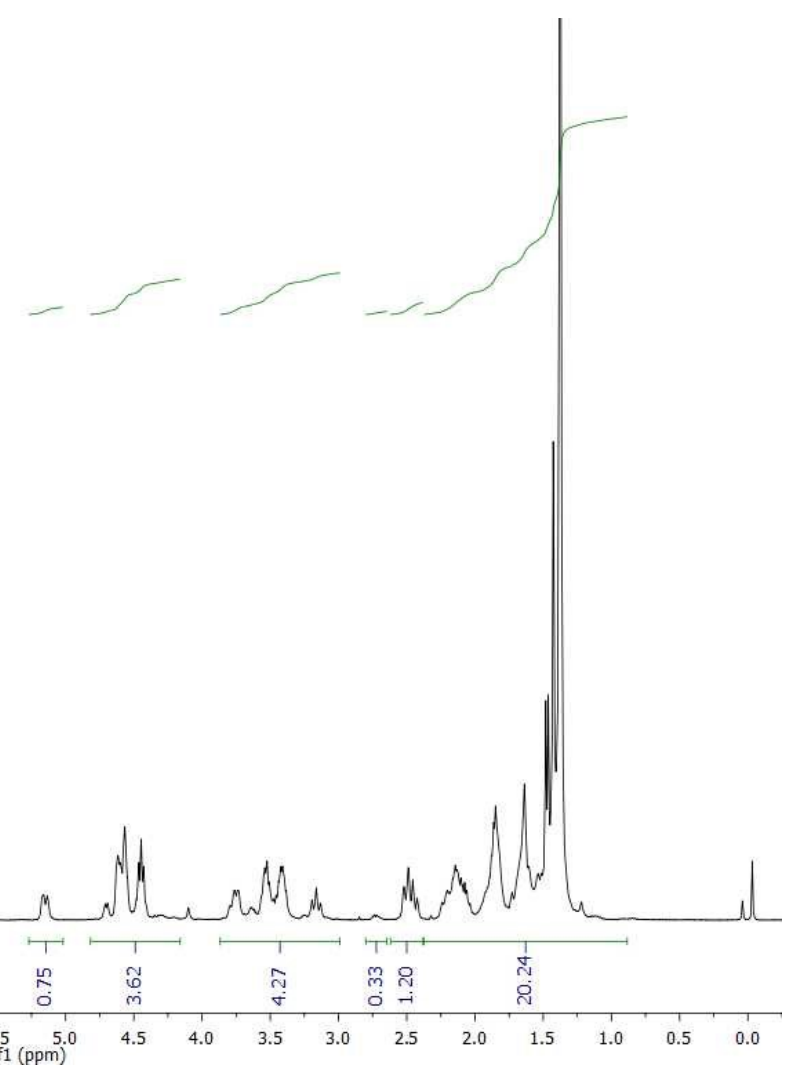

\begin{tabular}{|lll|}
\hline \multicolumn{1}{|c|}{ Parameter } & \multicolumn{1}{c|}{ Value } \\
1 & Title & 20150522 _ynmrs_400_JG-402_crude_rerun_2D-CARBON_01 \\
2 & Solvent & cdd3_insert \\
3 & Temperature & 25.0 \\
4 & Number of Scans & 14000 \\
5 & Relaxation Delay & 1.0000 \\
6 & Acquisition Time & 1.3631 \\
7 & Acquisition Date & $2015-05-22 T 00: 37: 02$ \\
8 & Spectrometer Frequency 100.53 \\
9 & Spectral Width & 24038.5 \\
10 & Lowest Frequency & -1451.2 \\
11 & Nucleus & $13 C$ \\
12 & Acquired Size & 32768 \\
13 & Spectral Size & 65536 \\
\hline
\end{tabular}

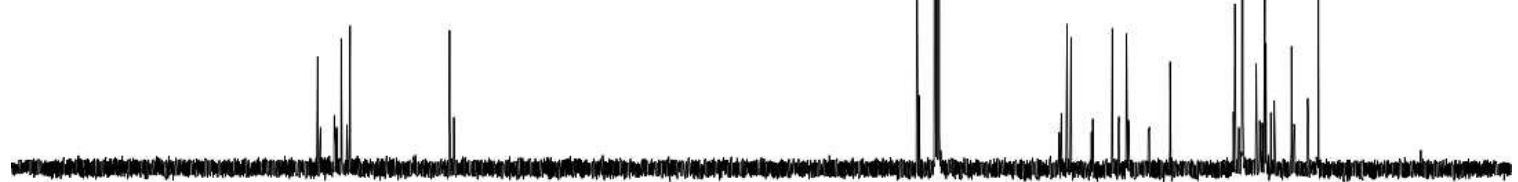

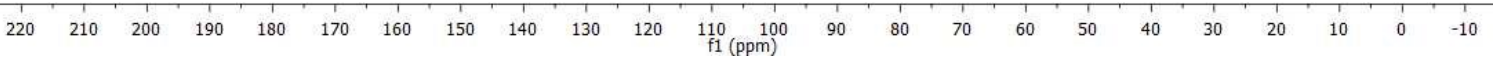




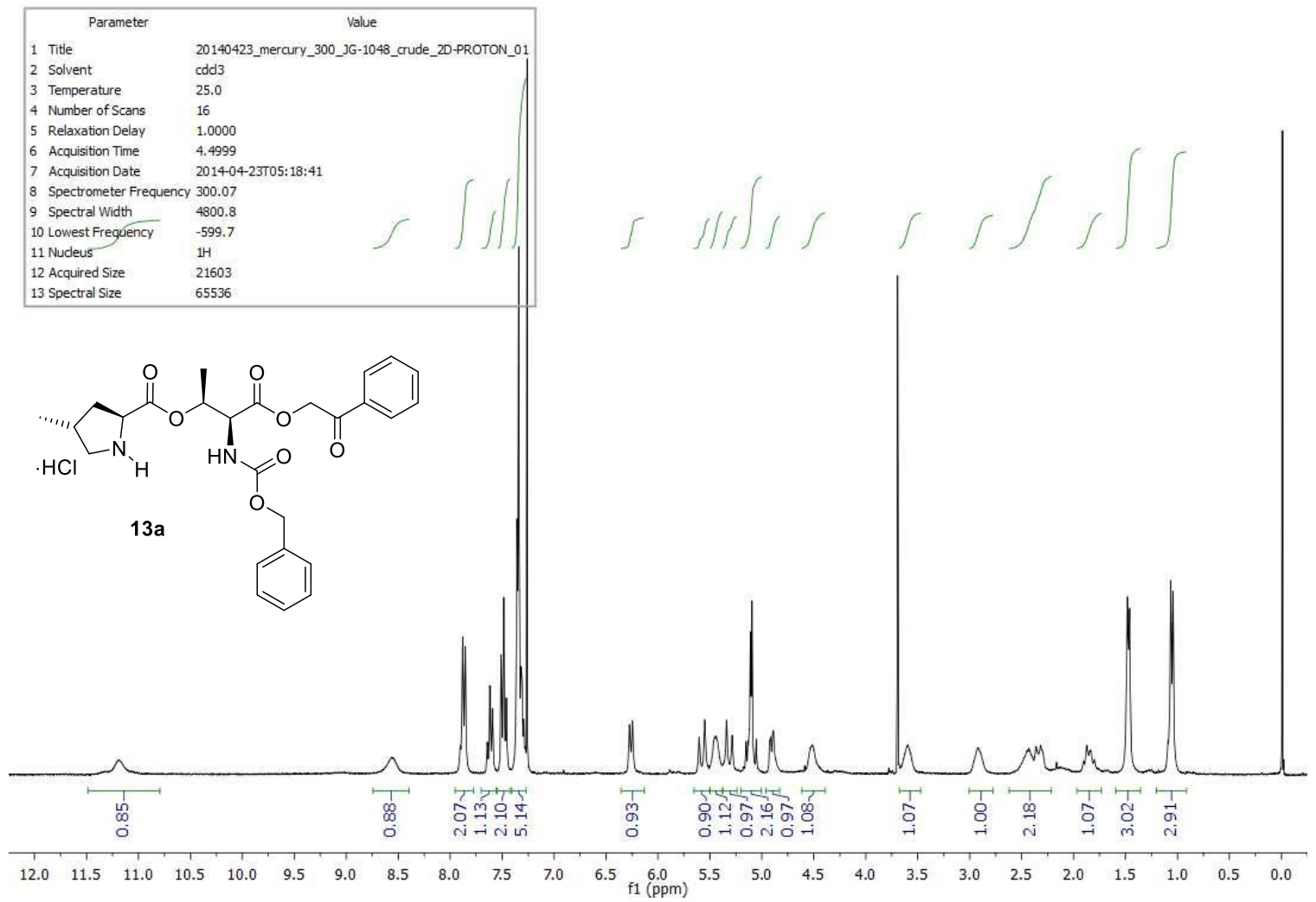

\begin{tabular}{|lll|}
\hline \multicolumn{2}{|c|}{ Parameter } & \multicolumn{1}{c|}{ Value } \\
1 & Tite & 20140423 mercury_300_JG-1048_crude_2D-CARBON_02 \\
2 & Solvent & cdd13 \\
3 & Temperature & 25.0 \\
4 & Number of Scans & 4000 \\
5 & Relaxation Delay & 0.5000 \\
6 & Acquisition Time & 2.0000 \\
7 & Acquisition Date & $2014.04-23 T 05: 38: 02$ \\
8 & Spectrometer Frequency 75.46 \\
9 & Spectral Width & 18867.9 \\
10 & Lowest Frequency & -1124.7 \\
11 & Nucleus & $13 \mathrm{C}$ \\
12 Acquired Size & 37736 \\
13 & Spectral Size & 131072 \\
\hline
\end{tabular}

$\begin{array}{llllllllllllllllllllllllll}230 & 220 & 210 & 200 & 190 & 180 & 170 & 160 & 150 & 140 & 130 & 120 & \begin{array}{c}110 \\ \mathrm{f} 1(\mathrm{ppm})\end{array} & 100 & 90 & 80 & 70 & 60 & 50 & 40 & 30 & 20 & 10 & 0 & -10\end{array}$ 


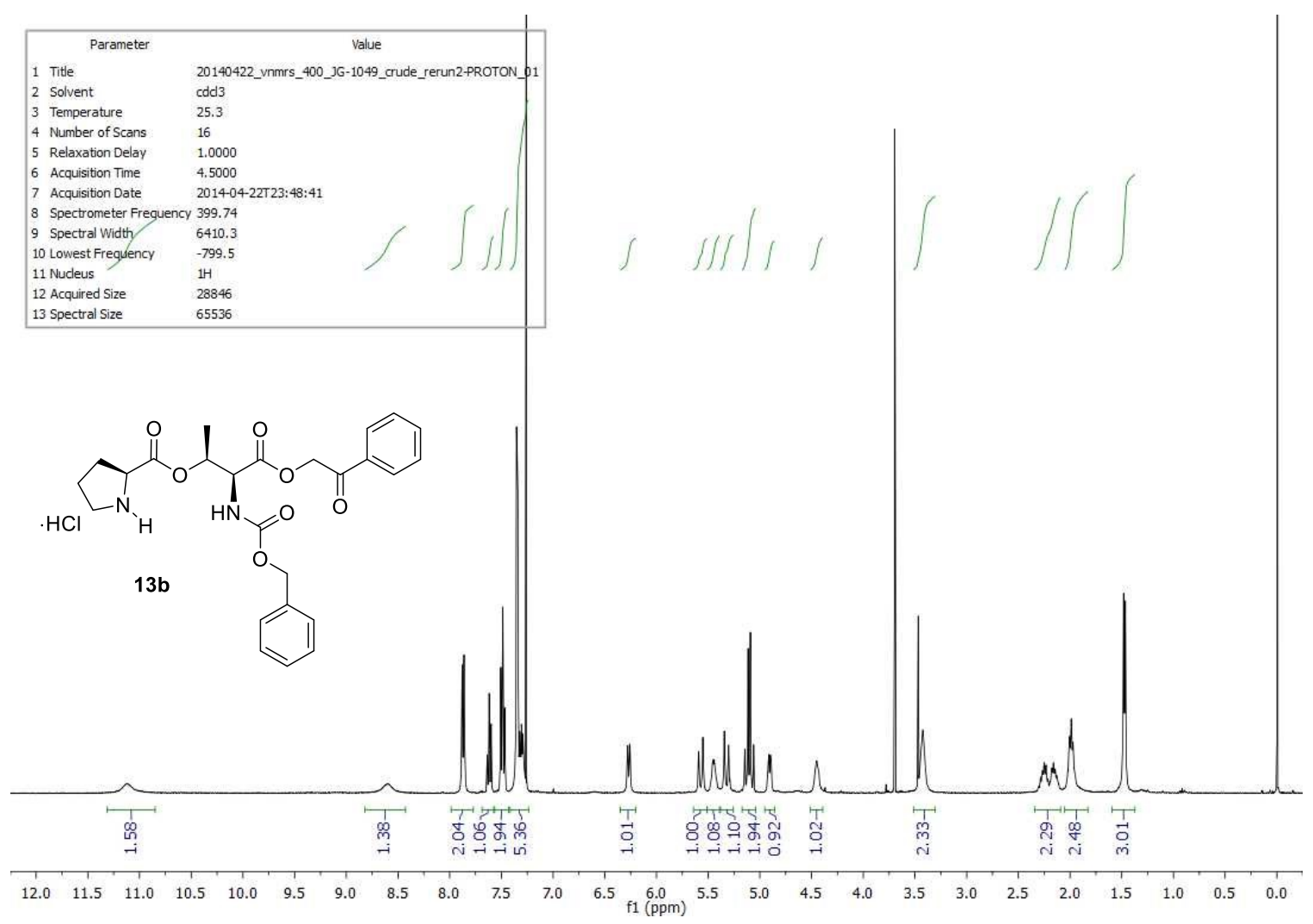

\begin{tabular}{|c|c|}
\hline Parameter & Value \\
\hline 1 Title & 20140423_ynmrs_400_JG-1049_crude_rerun2-CARBON_01 \\
\hline 2 Solvent & cdd3 \\
\hline 3 Temperature & 25.3 \\
\hline 4 Number of Scans & 5000 \\
\hline 5 Relaxation Delay & 1.0000 \\
\hline 6 Acquisition Time & 1.3631 \\
\hline 7 Acquisition Date & 2014-04-23T00:29:07 \\
\hline 8 Spectrometer Frequency & 100.53 \\
\hline 9 Spectral Width & 24038.5 \\
\hline 10 Lowest Frequency & -1448.5 \\
\hline 11 Nudleus & $13 \mathrm{C}$ \\
\hline 12 Acquired Size & 32768 \\
\hline 13 Spectral Size & 65536 \\
\hline
\end{tabular}

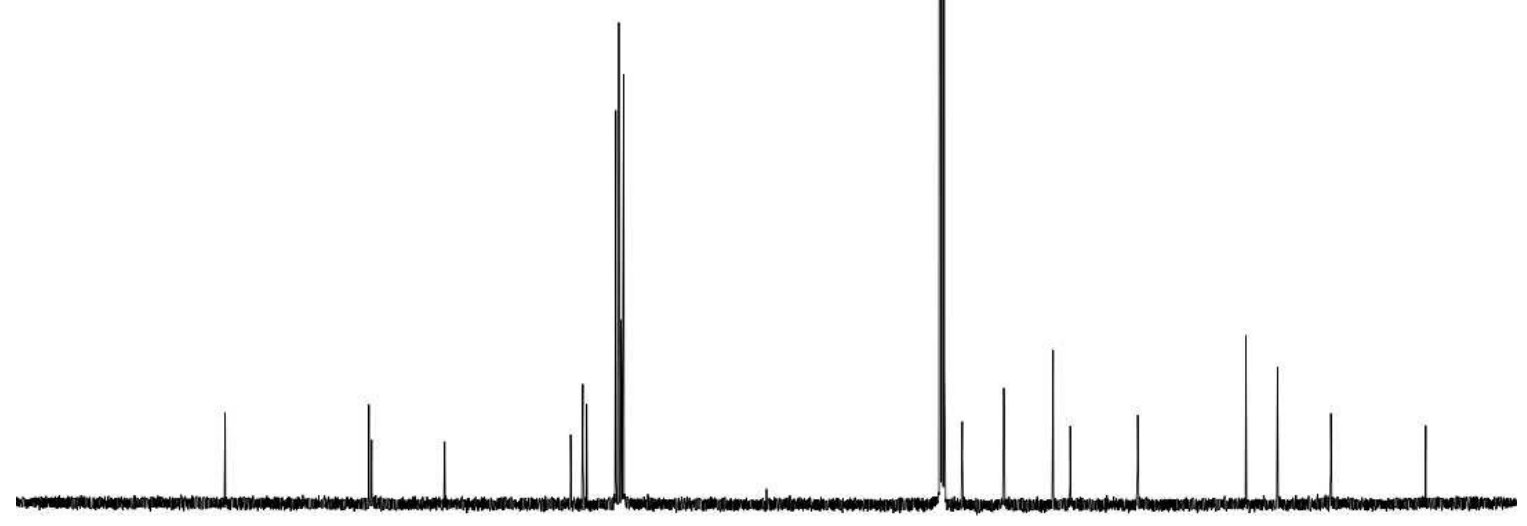

\begin{tabular}{lllllllllllllllllllllllll}
\hline & 220 & 210 & 200 & 190 & 180 & 170 & 160 & 150 & 140 & 130 & 120 & 110 & 100 & 90 & 80 & 70 & 60 & 50 & 40 & 30 & 20 & 10 & 0 & -10
\end{tabular} 

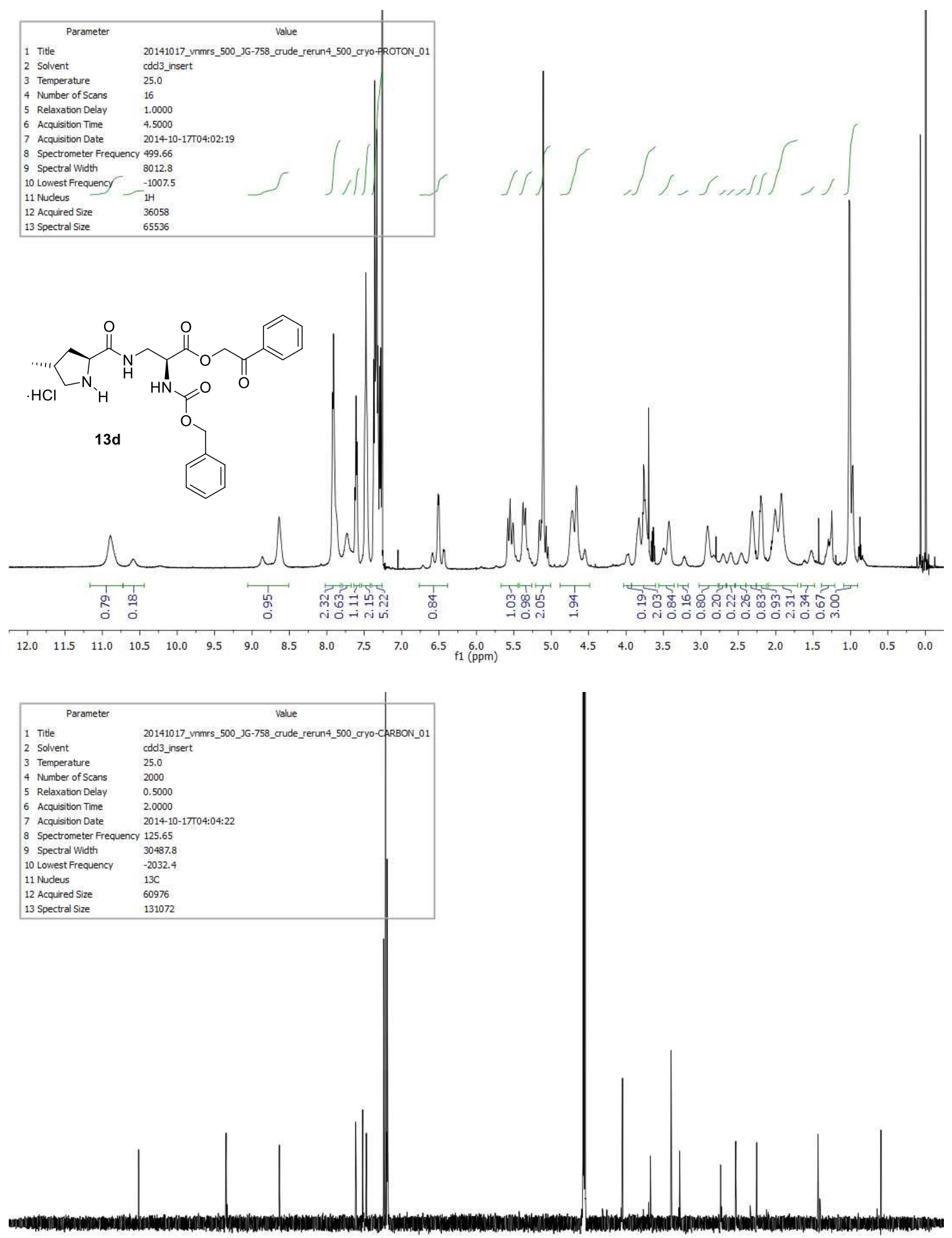

\begin{tabular}{lllllllllllllllllllllllll}
\hline 220 & 210 & 200 & 190 & 180 & 170 & 160 & 150 & 140 & 130 & 120 & 110 & 100 & 90 & 80 & 70 & 60 & 50 & 40 & 30 & 20 & 10 & 0 & -10
\end{tabular} 

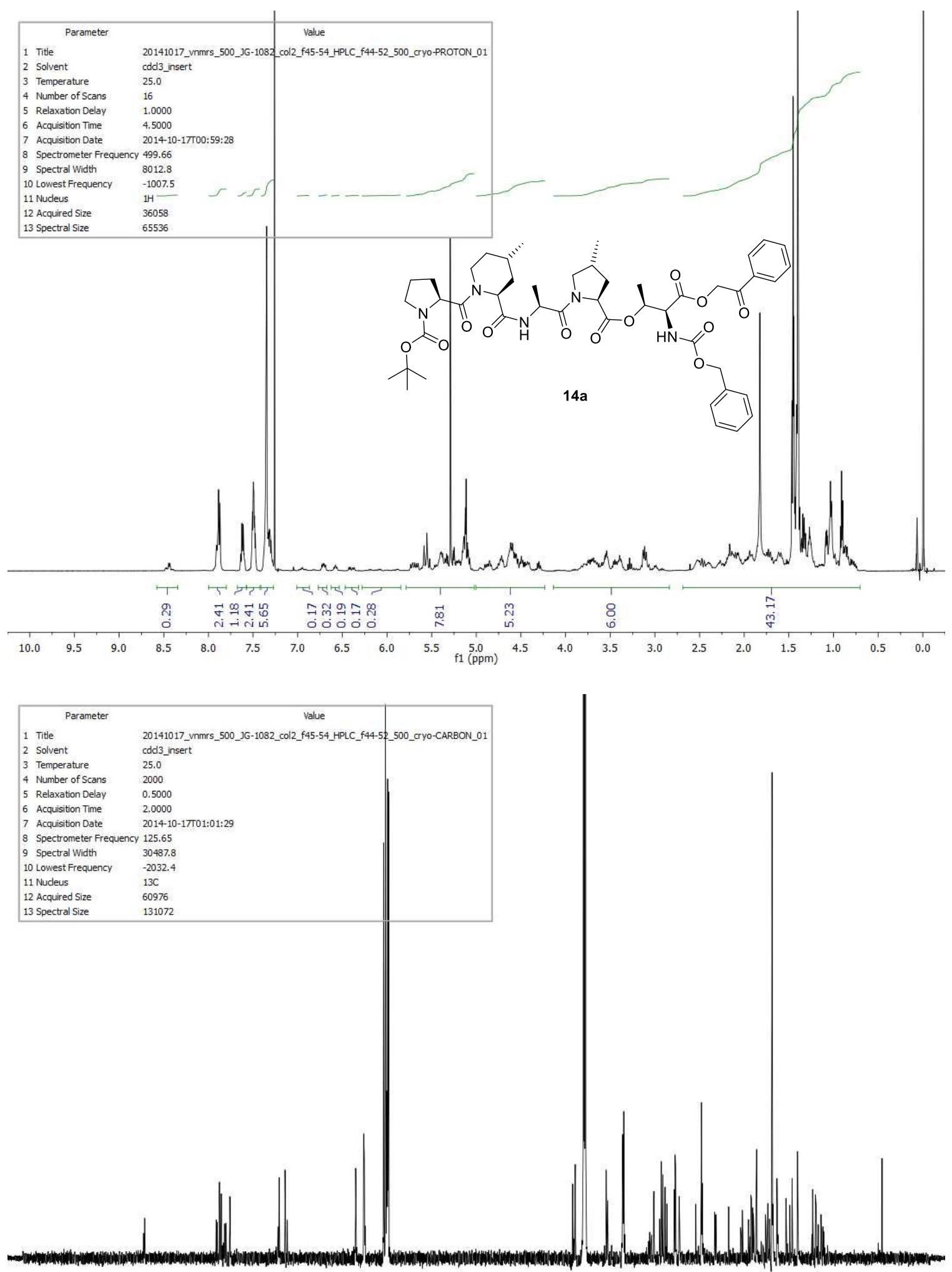

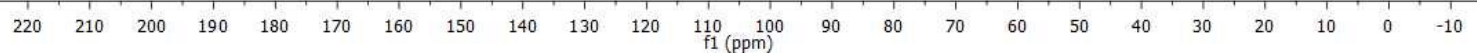



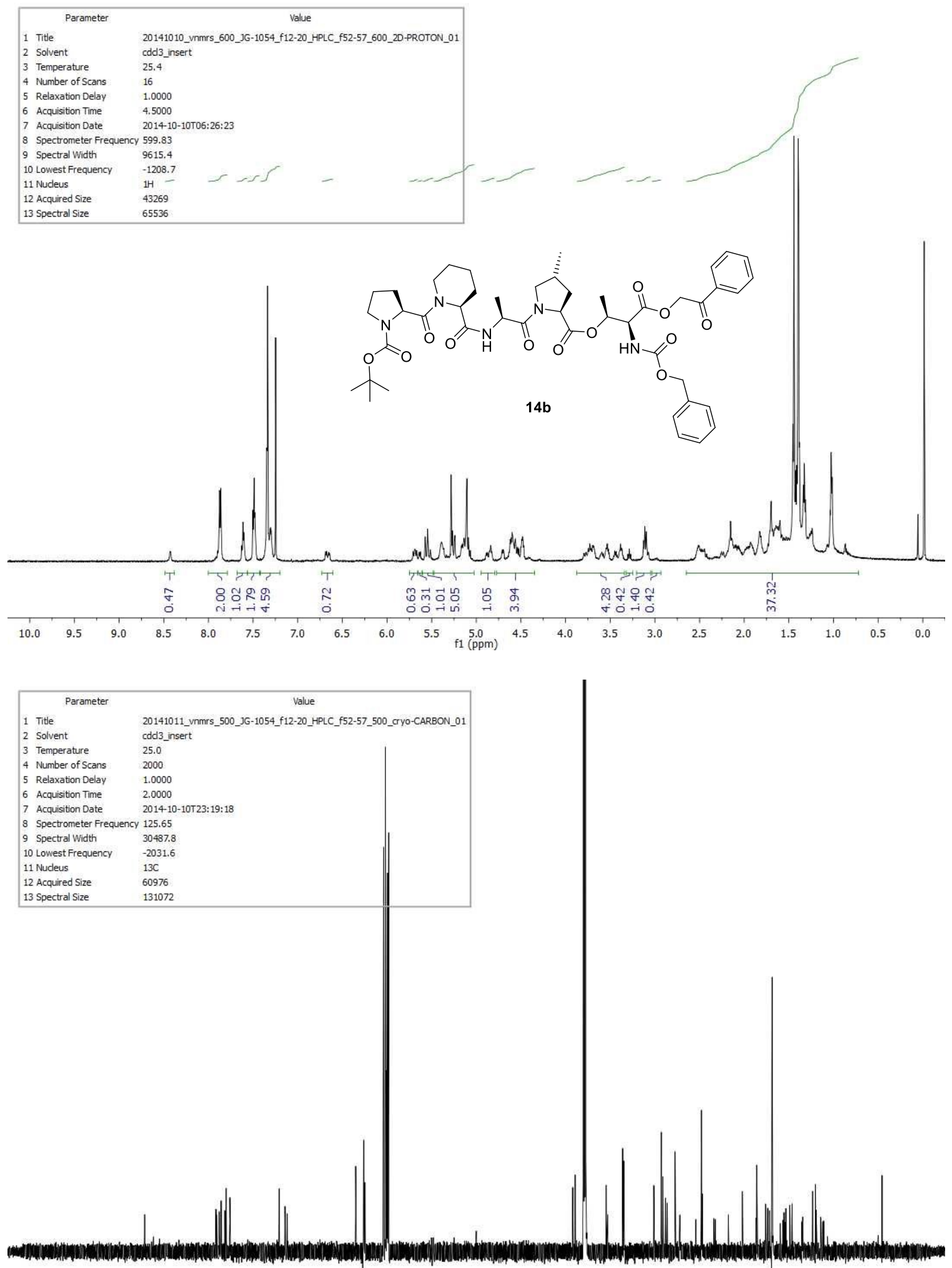

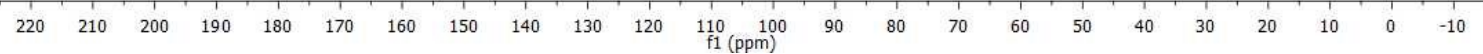



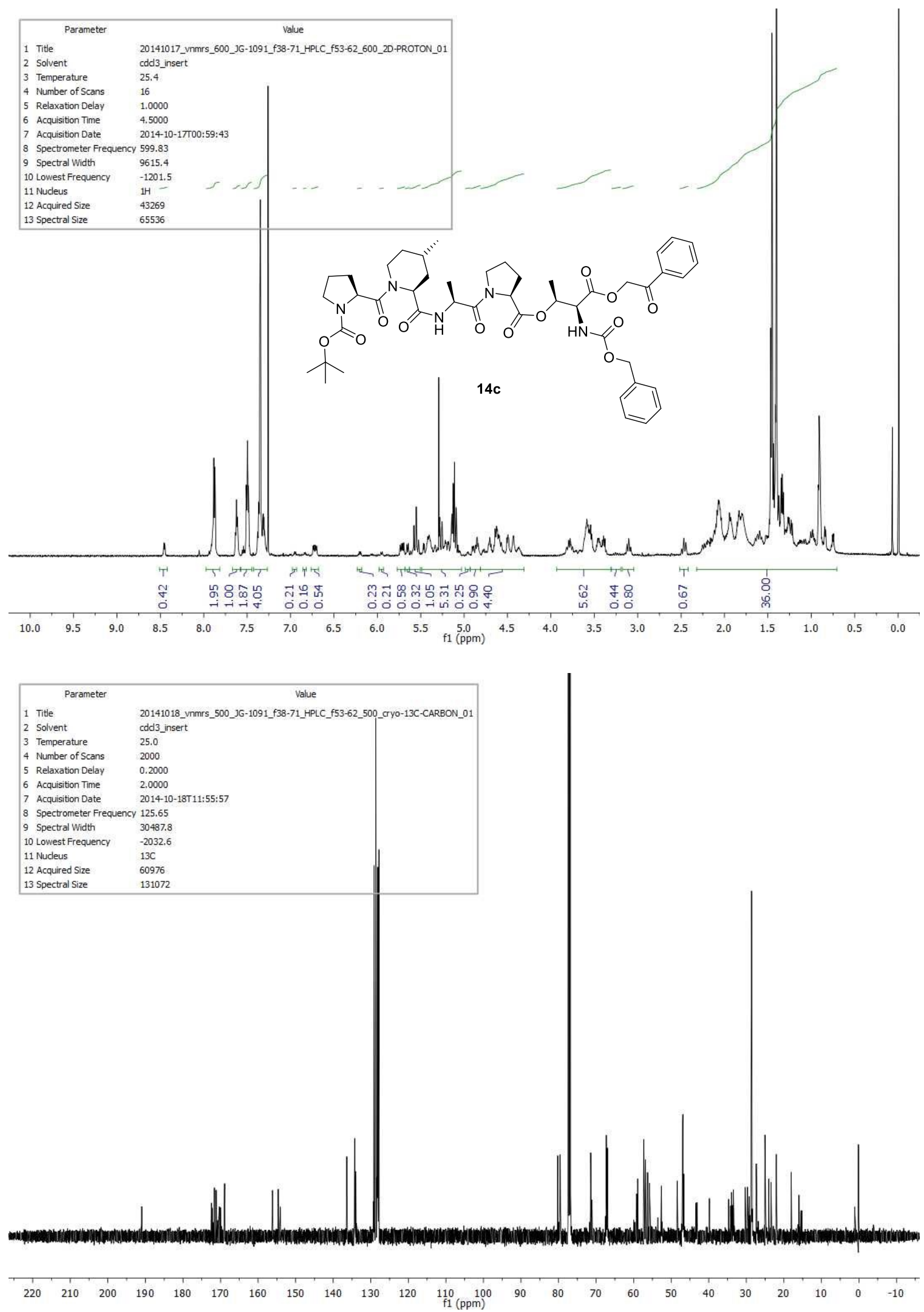

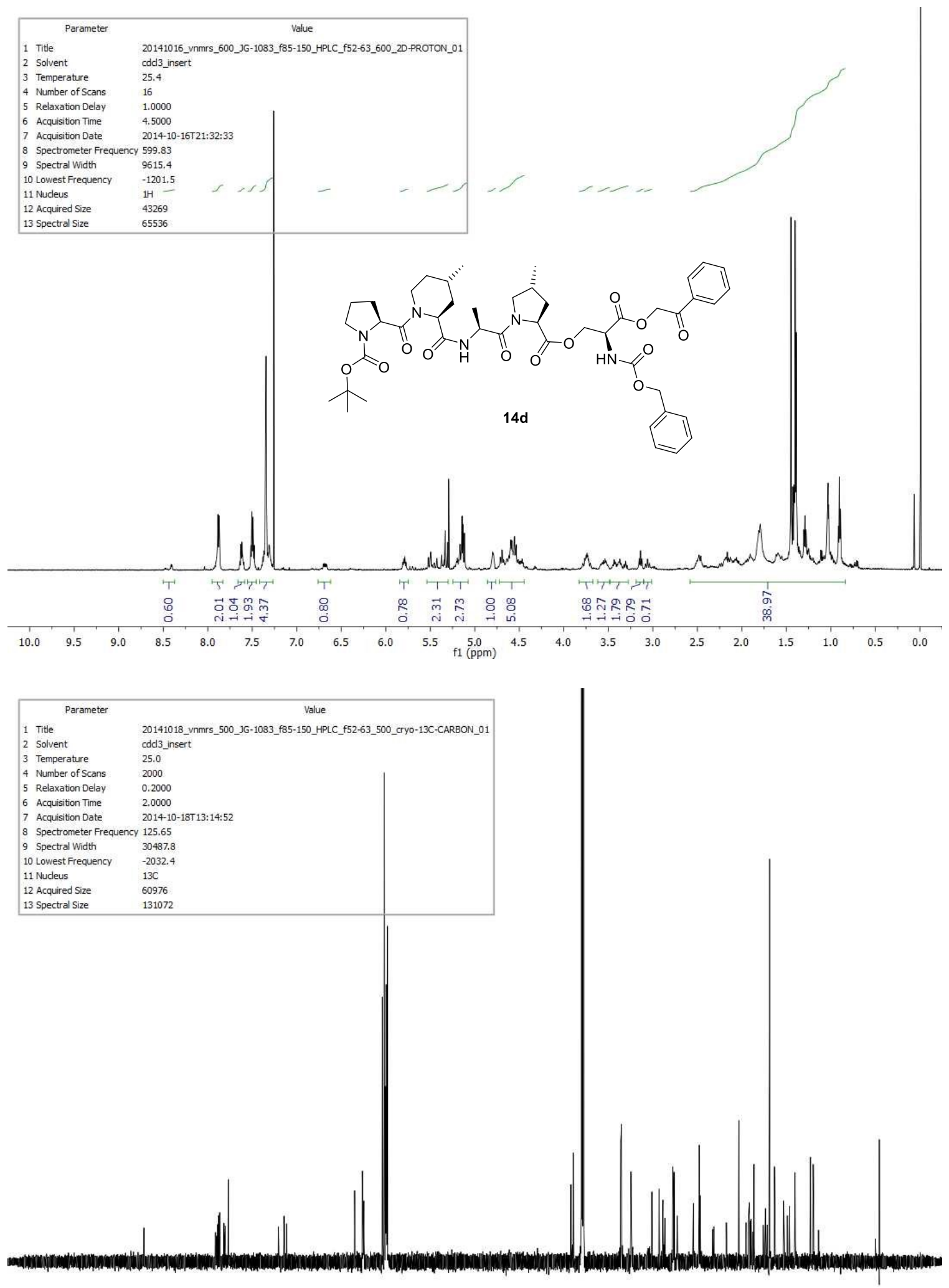

$\begin{array}{llllllllllllllllllllllll}220 & 210 & 200 & 190 & 180 & 170 & 160 & 150 & 140 & 130 & 120 & 110 & 100 & 90 & 80 & 70 & 60 & 50 & 40 & 30 & 20 & 10 & 0 & -10\end{array}$ 

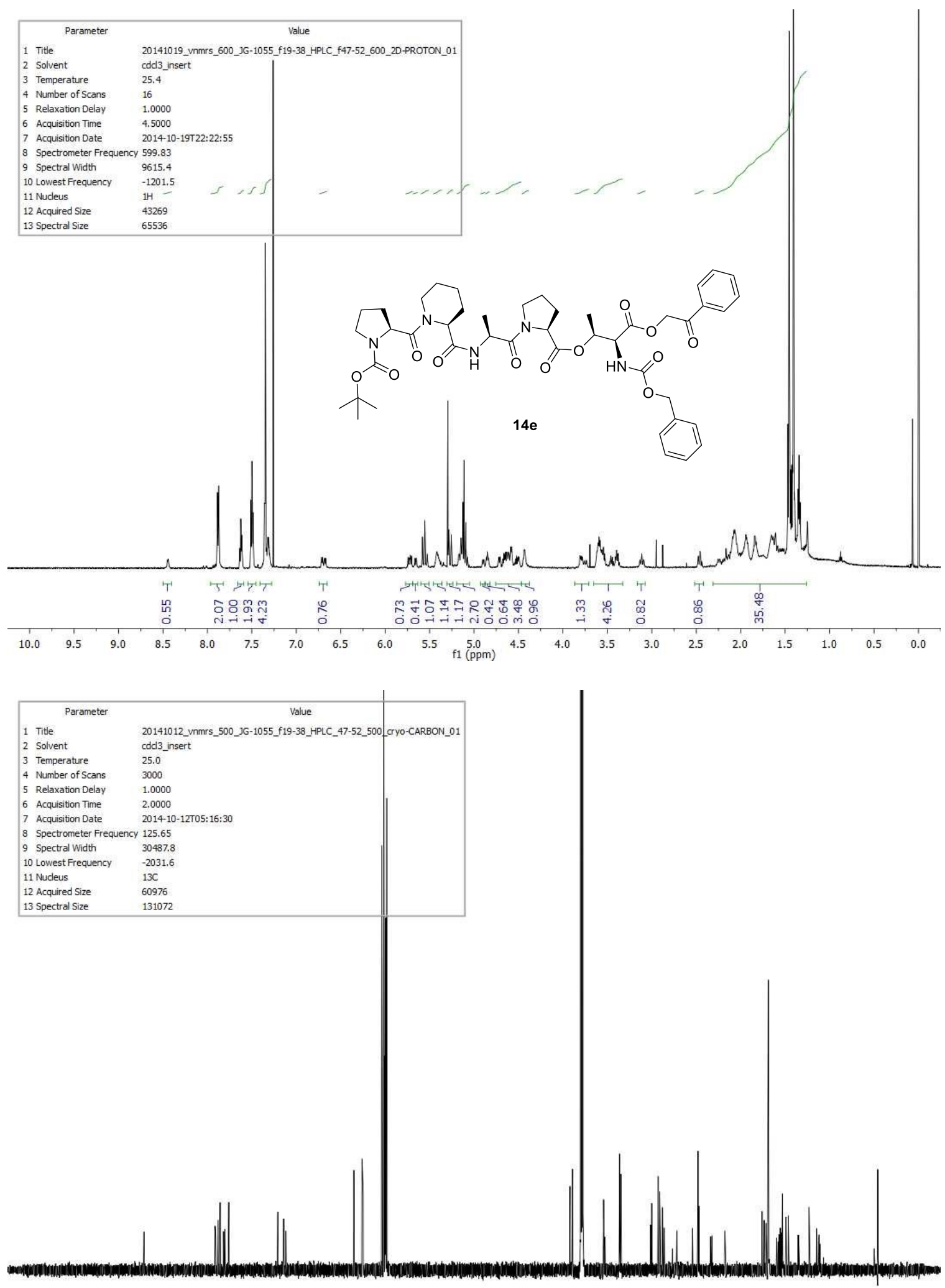

$\begin{array}{lllllllllllllllllllllllllll}220 & 210 & 200 & 190 & 180 & 170 & 160 & 150 & 140 & 130 & 120 & \underset{\mathrm{f} 1}{110}(\mathrm{ppm}) & 100 & 90 & 80 & 70 & 60 & 50 & 40 & 30 & 20 & 10 & 0 & -10\end{array}$ 

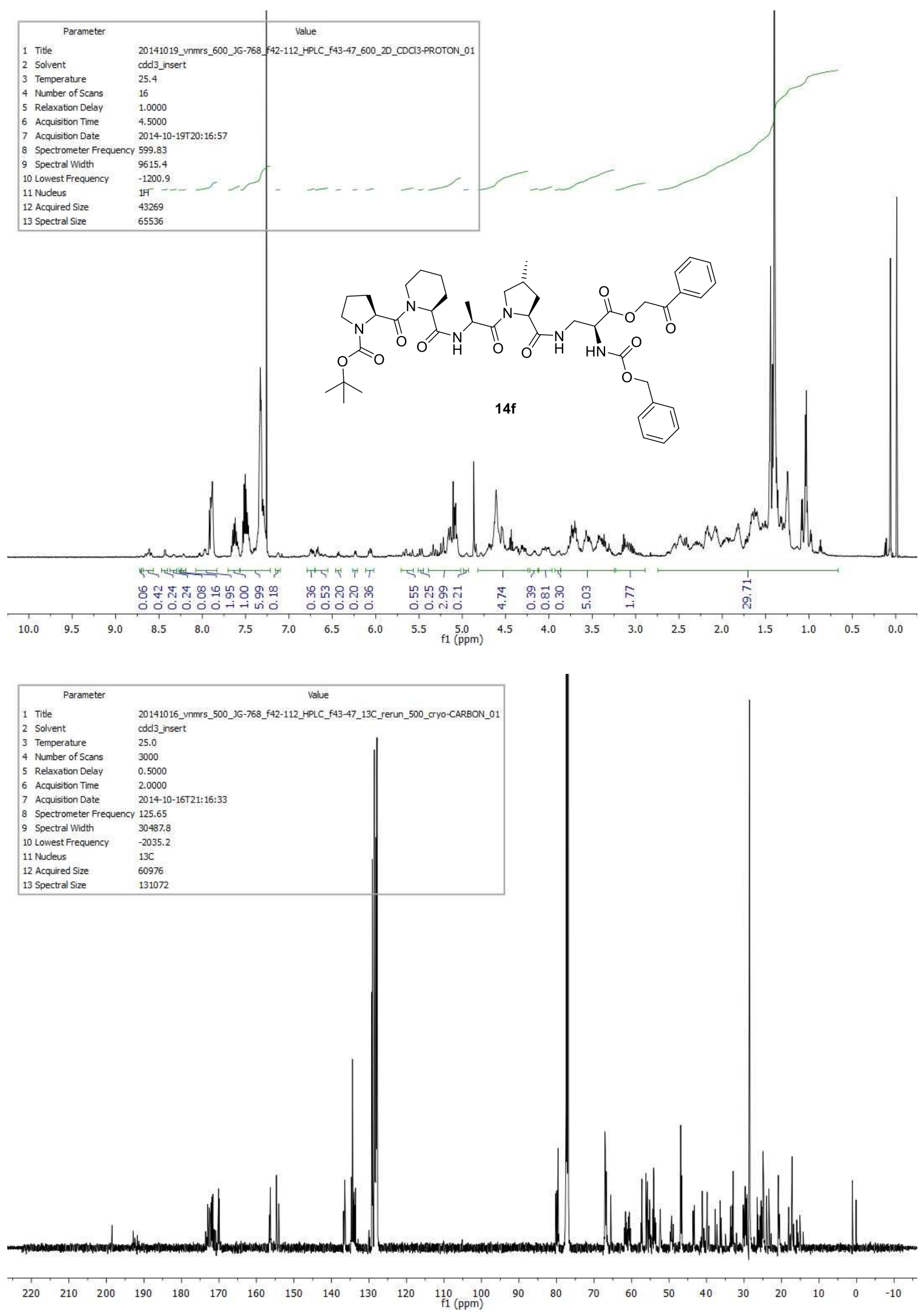

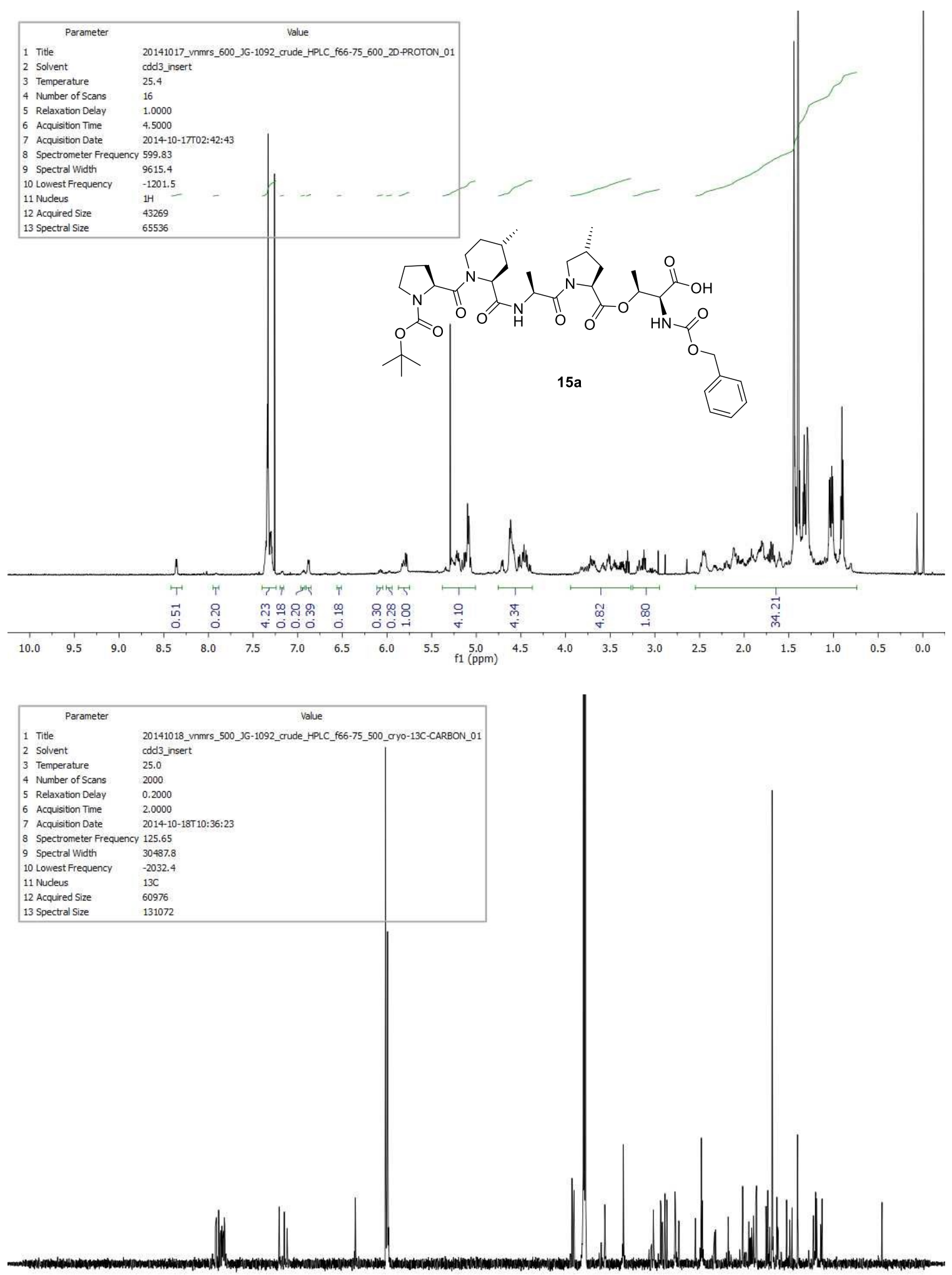

\begin{tabular}{|lllllllllllllllllllllllllll}
220 & 210 & 200 & 190 & 180 & 170 & 160 & 150 & 140 & 130 & 120 & $\underset{\mathrm{f} 1}{110}(\mathrm{ppm})$ & 100 & 90 & 80 & 70 & 60 & 50 & 40 & 30 & 20 & 10 & 0 & -10
\end{tabular} 

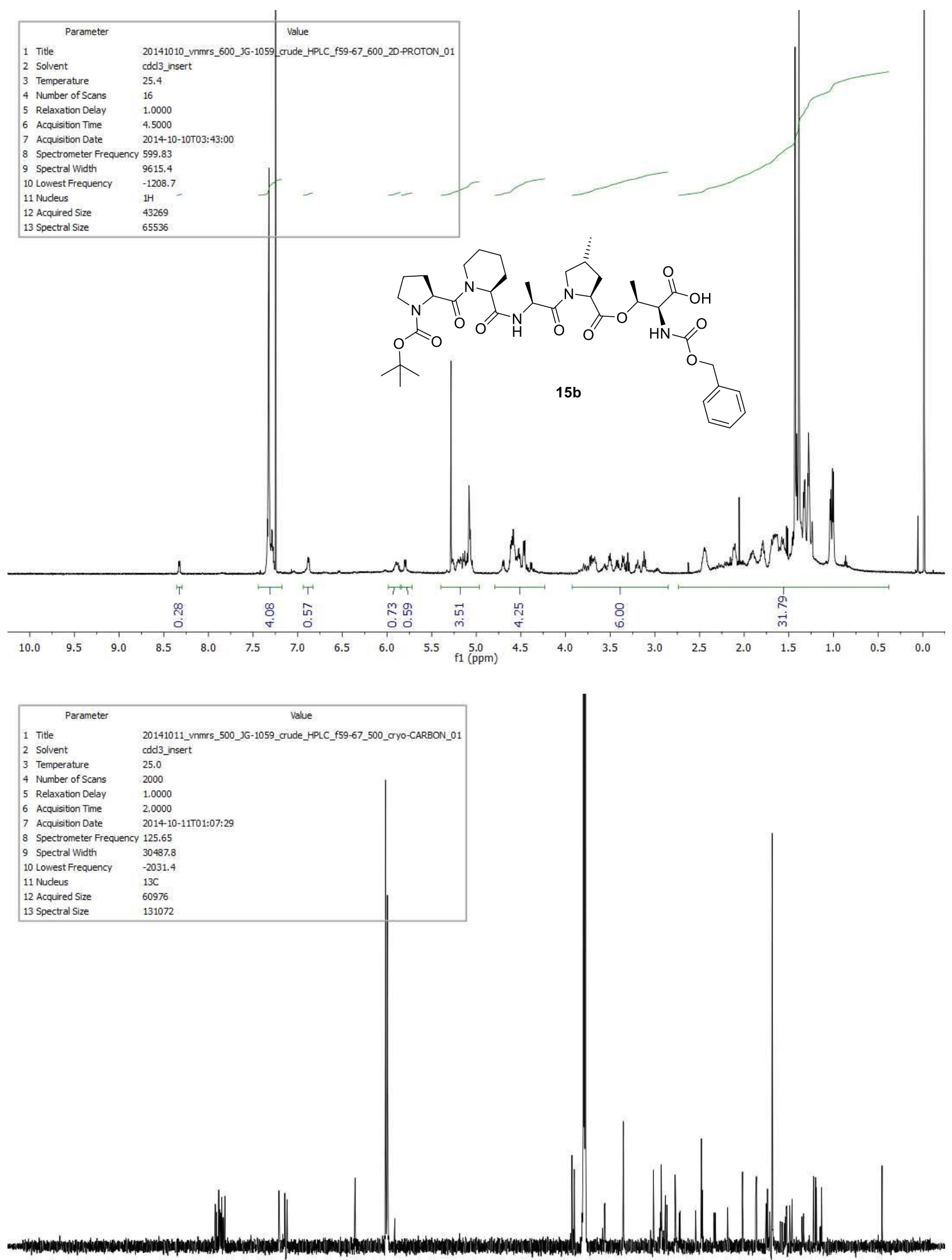

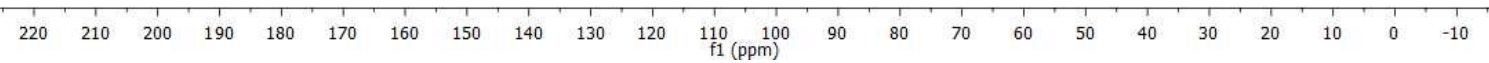



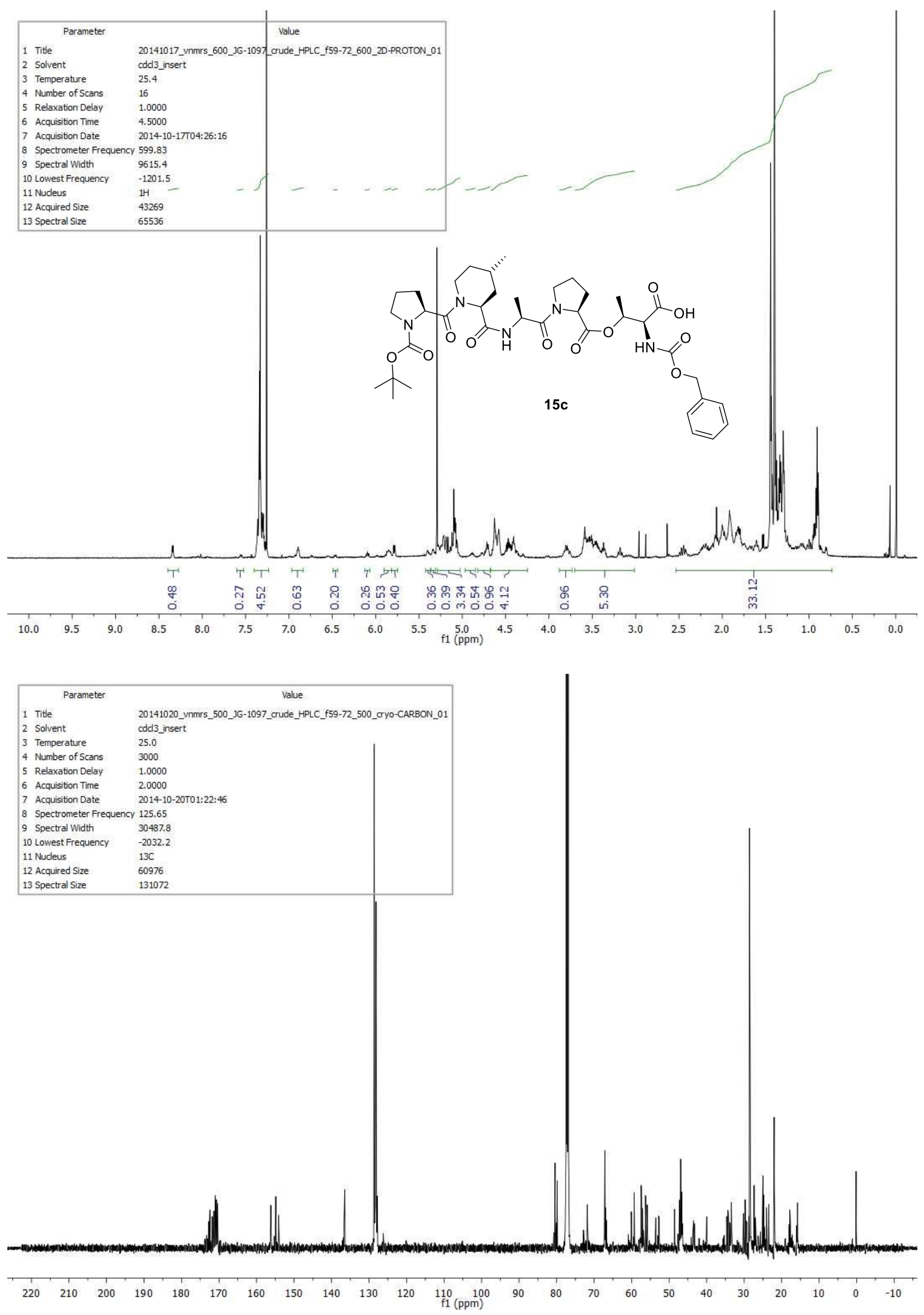

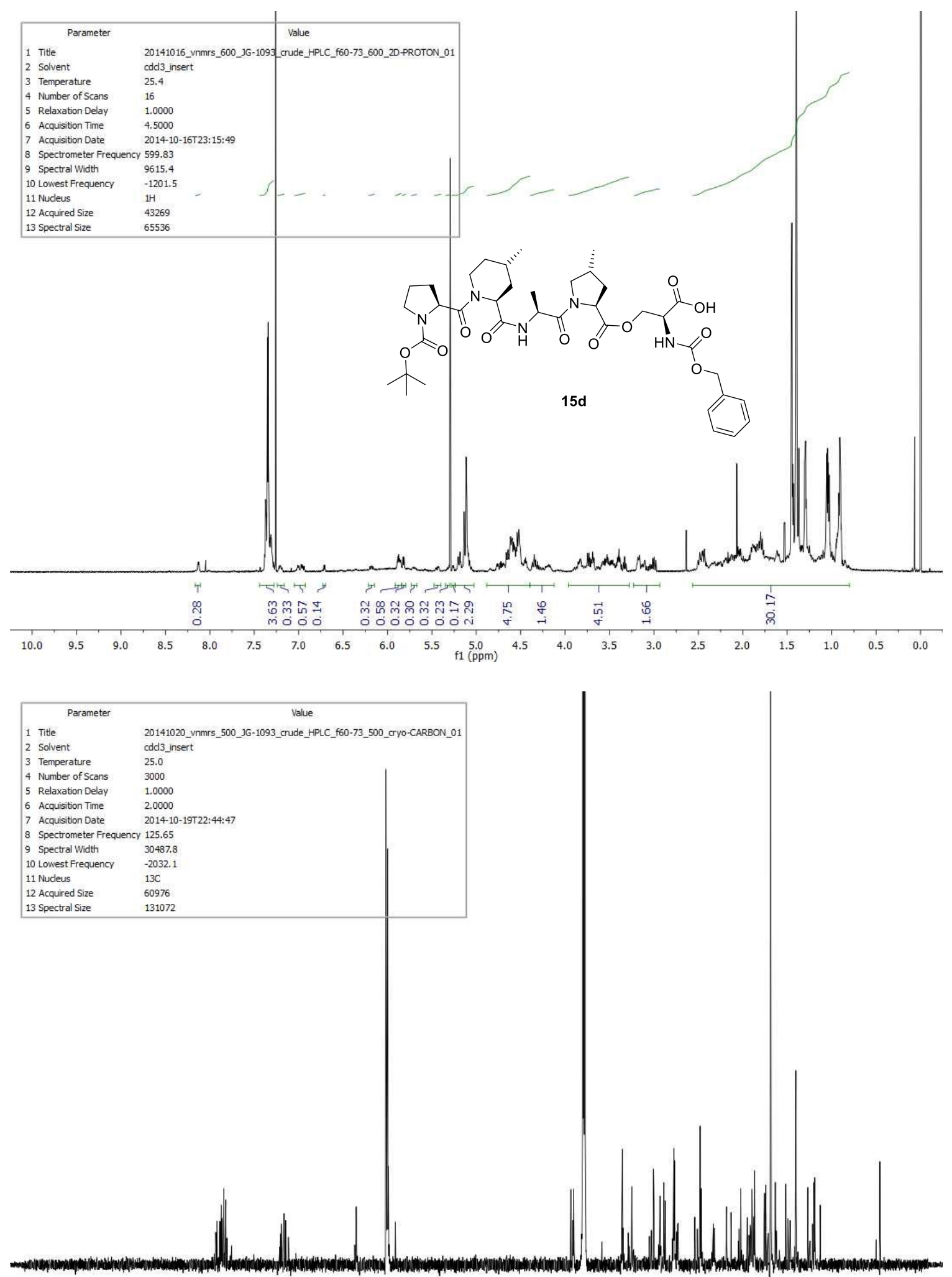

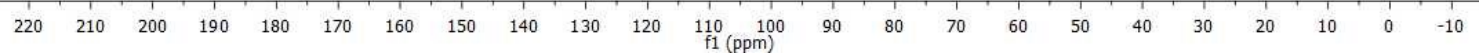



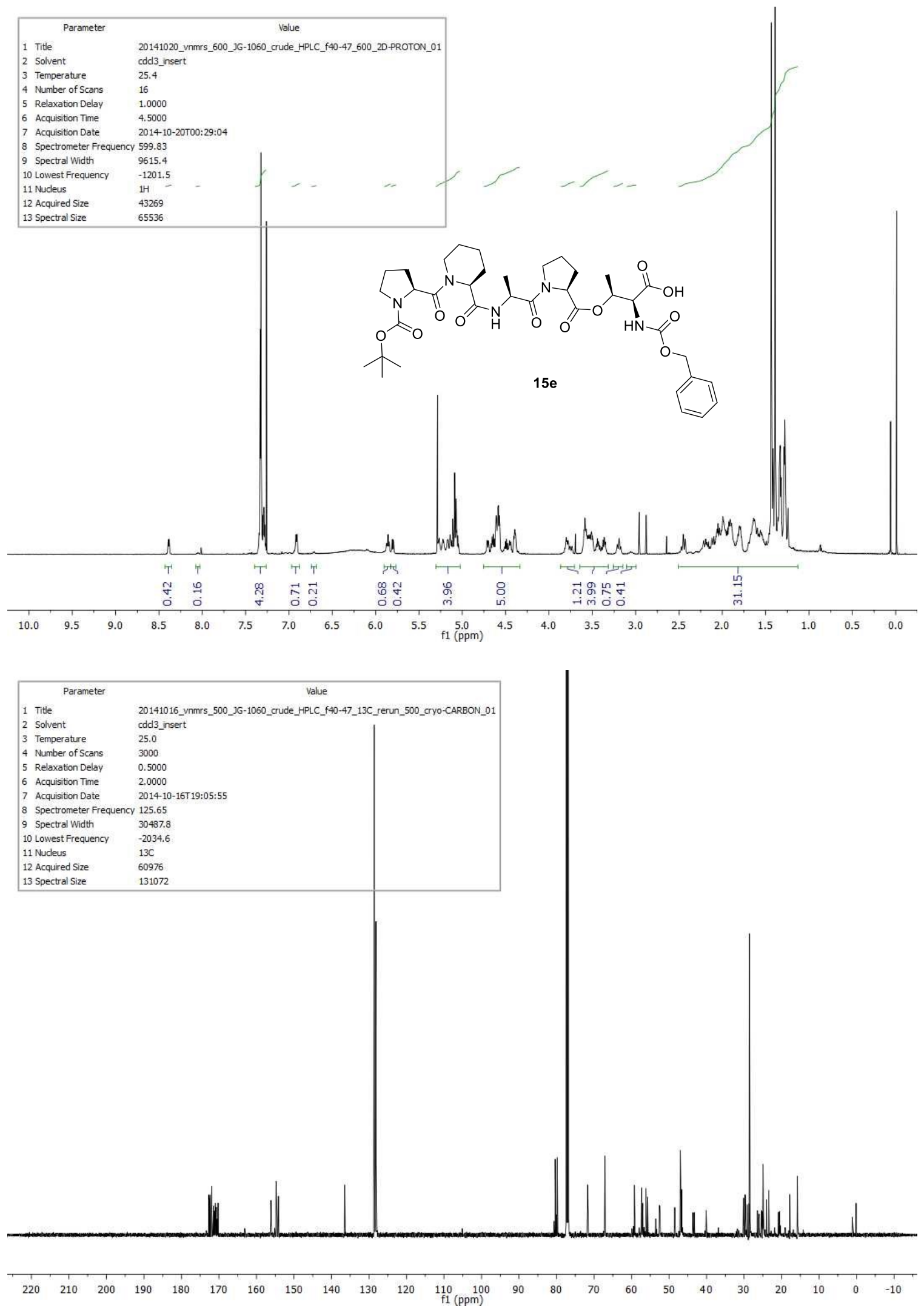

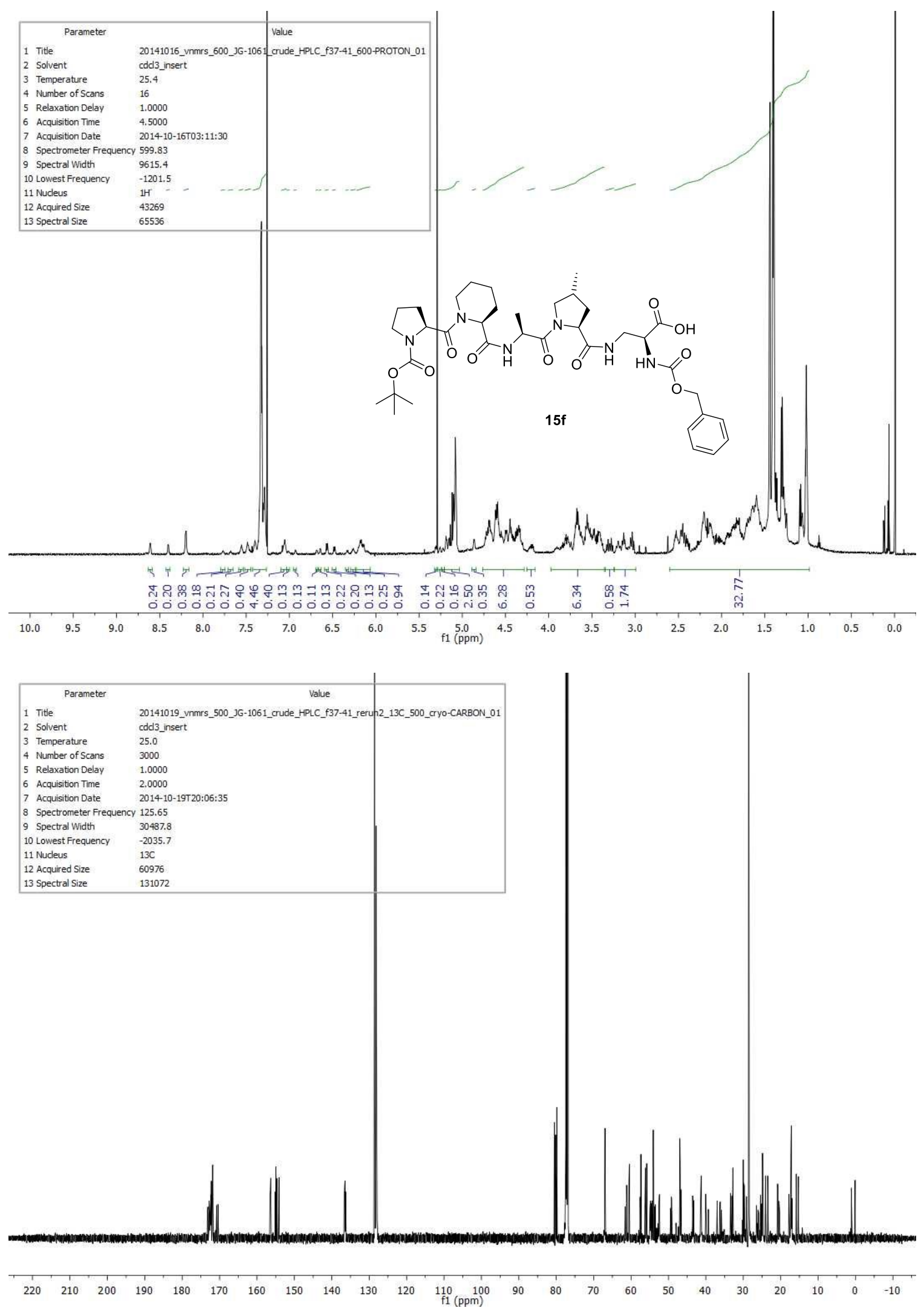


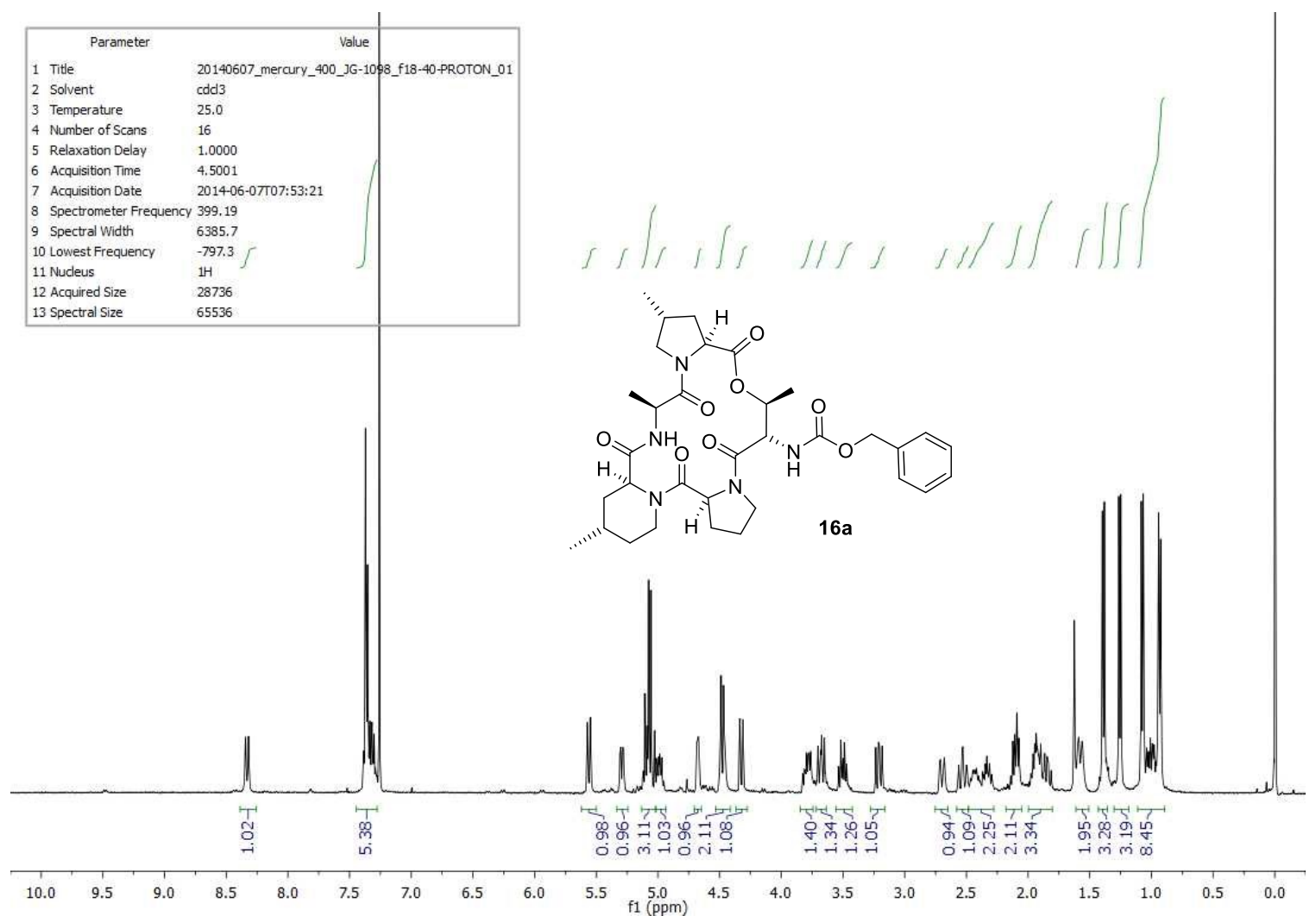

\begin{tabular}{|lll|}
\hline \multicolumn{1}{|c|}{ Parameter } & \multicolumn{1}{c|}{ Value } \\
1 & Tite & 20140610 mercury_400_JG-1098_f18-40_13C-CARBON_01 \\
2 & Solvent & cdd3 \\
3 & Temperature & 25.0 \\
4 & Number of Scans & 1800 \\
5 & Relaxation Delay & 1.0000 \\
6 & Acquisition Time & 2.0000 \\
7 & Acquisition Date & $201406-10004: 03: 50$ \\
8 & Spectrometer Frequency & 100.39 \\
9 & Spectral Width & 25125.6 \\
10 & Lowest Frequency & -1505.9 \\
11 & Nucleus & $13 \mathrm{C}$ \\
12 & Acquired Size & 50251 \\
13 & Spectral Size & 131072 \\
\hline
\end{tabular}

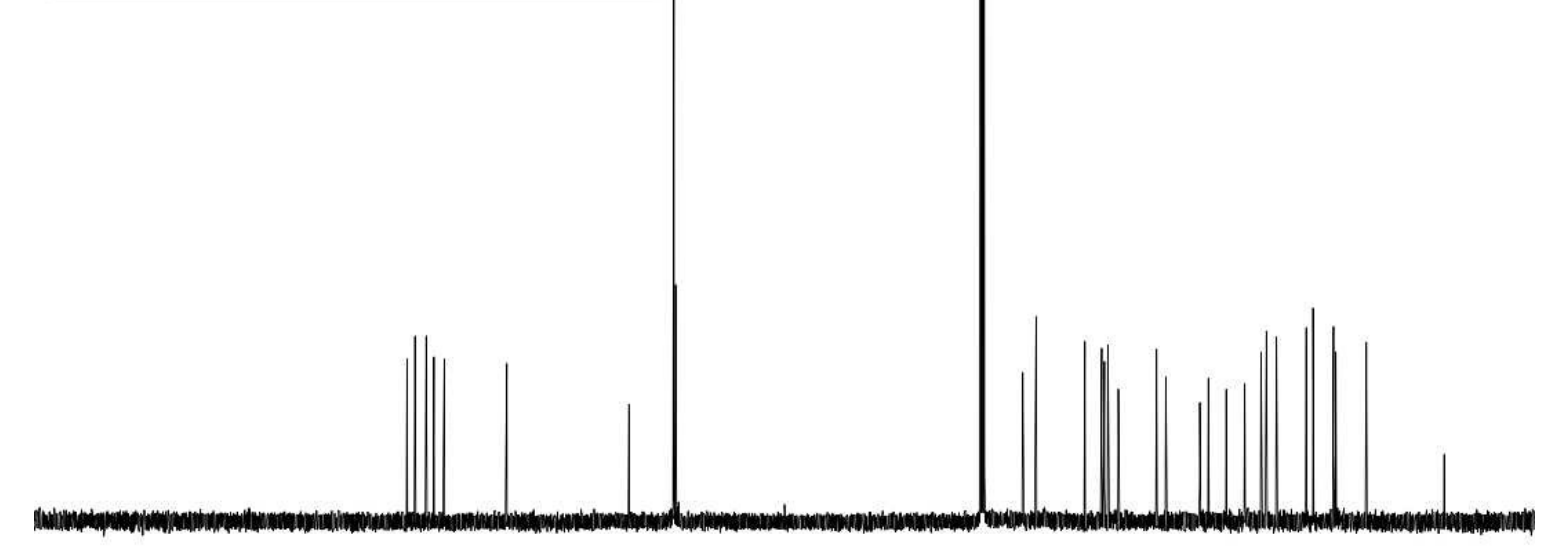

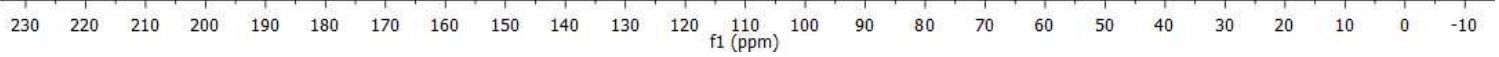



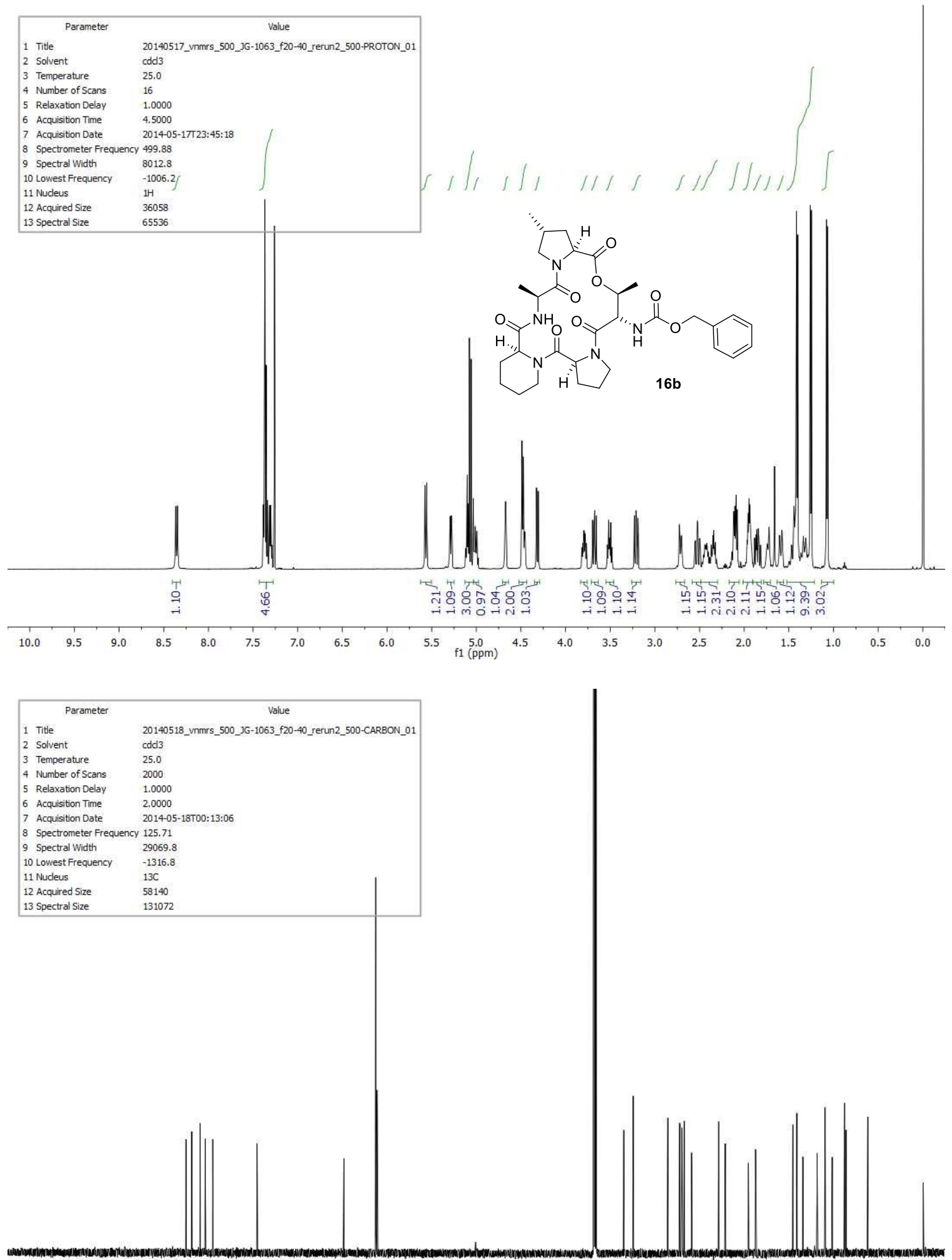

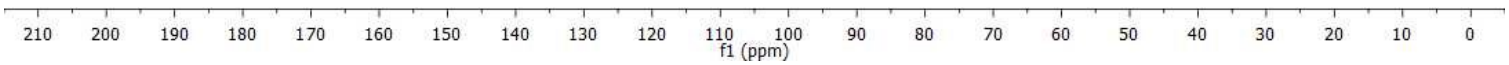



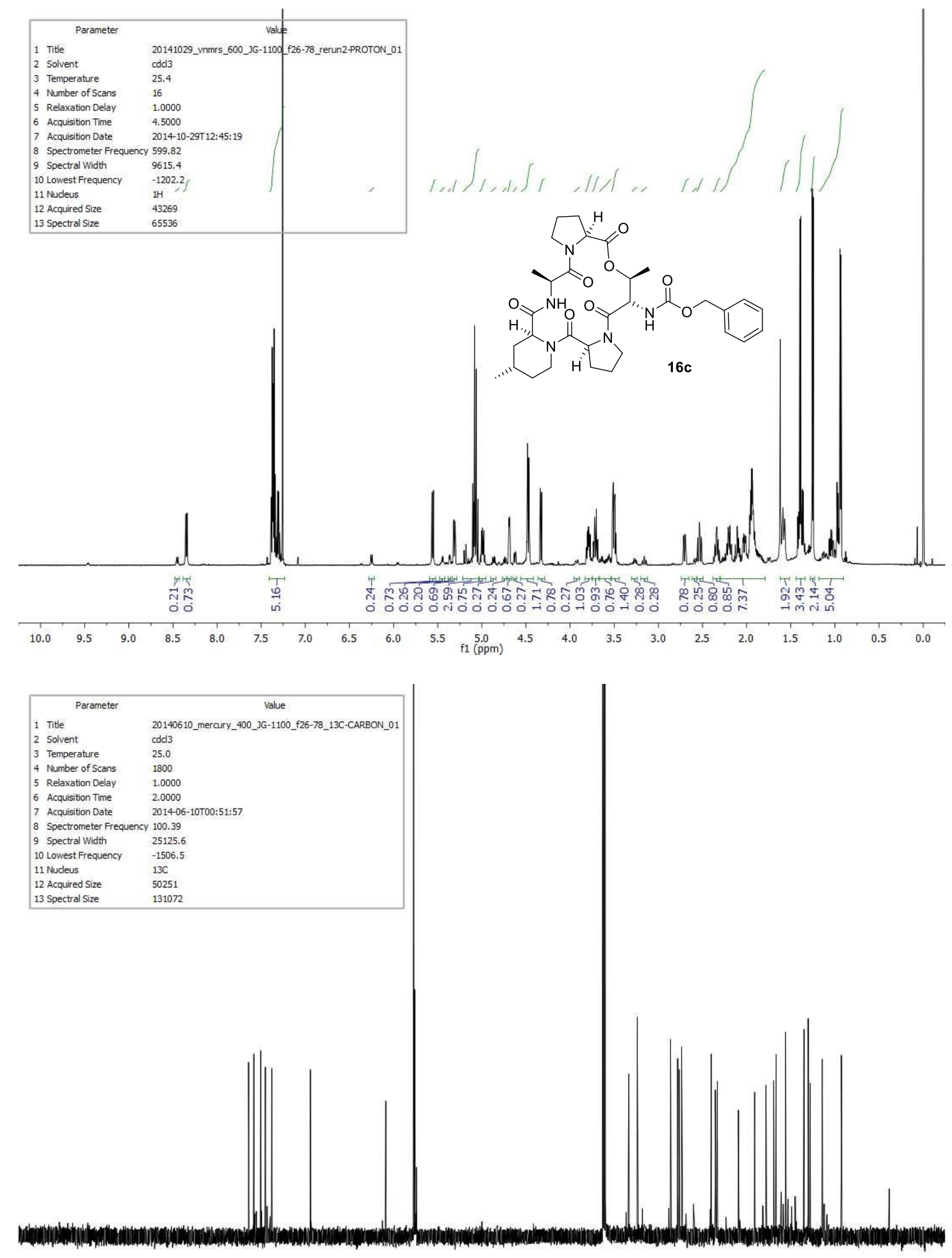

$\begin{array}{llllllllllllllllllllllllllllll} & 130 & 220 & 210 & 200 & 190 & 180 & 170 & 160 & 150 & 140 & 130 & 120 & 110 & 100 & 90 & 80 & 70 & 60 & 50 & 40 & 30 & 20 & 10 & 0 & -10\end{array}$ 


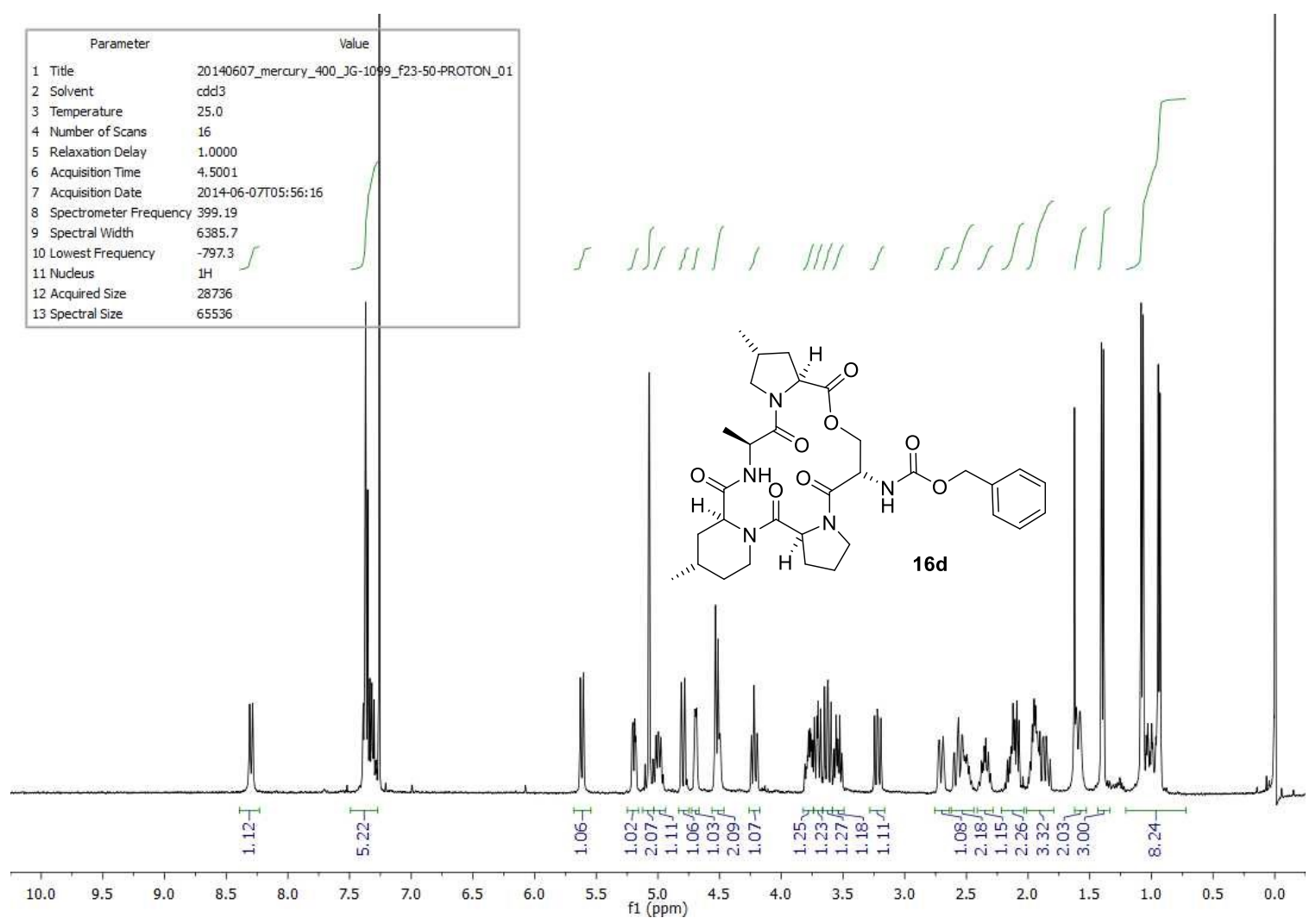

\begin{tabular}{|lll|}
\hline \multicolumn{1}{|c|}{ Parameter } \\
1 & Title & 20140610 mercury_400_JG-1099_f23-50_13C-CARBON_01 \\
2 & Solvent & cdd3 \\
3 & Temperature & 25.0 \\
4 & Number of Scans & 1800 \\
5 & Relaxation Delay & 1.0000 \\
6 & Acquisition Time & 2,0000 \\
7 & Acquisition Date & $2014.06-10$ T02:25:57 \\
8 & Spectrometer Frequency & 100.39 \\
9 & Spectral Width & 25125.6 \\
10 & Lowest Frequency & -1505.7 \\
11 Nucleus & 13 C \\
12 Acquired Size & 50251 \\
13 Spectral Size & 131072 \\
\hline
\end{tabular}

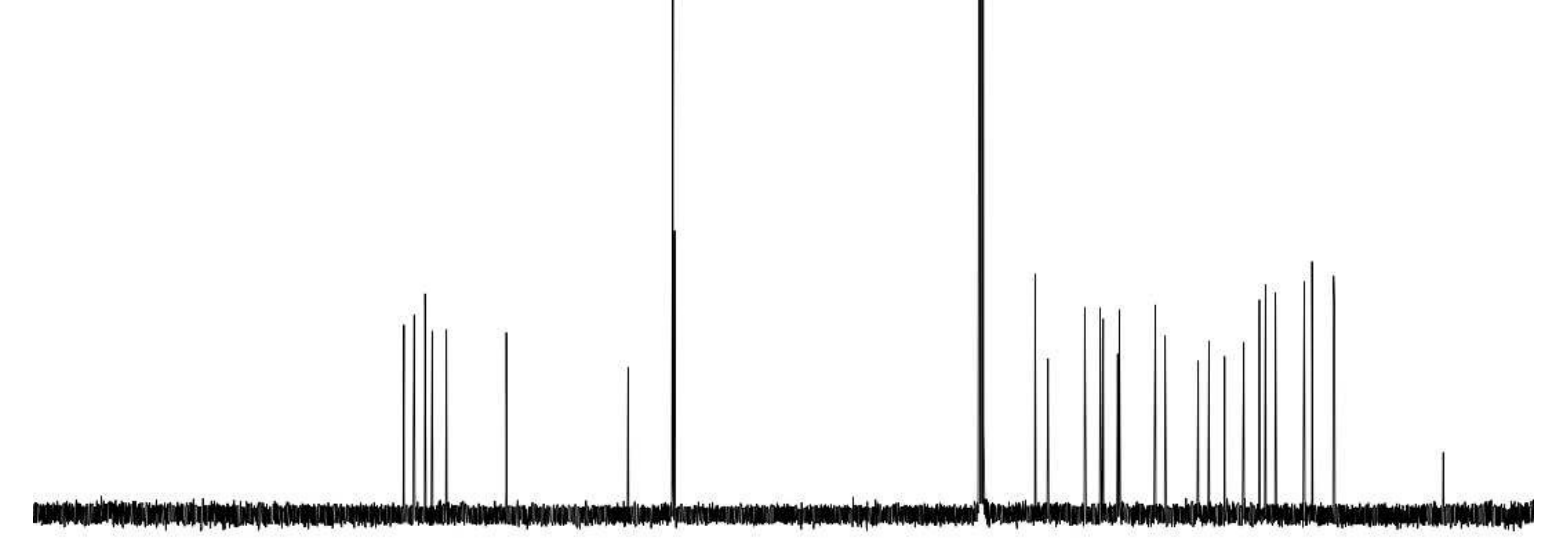

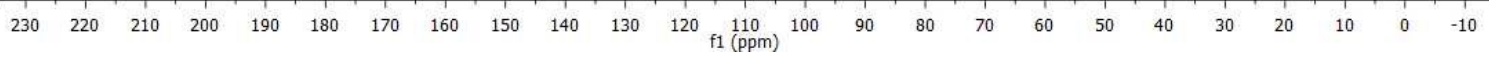



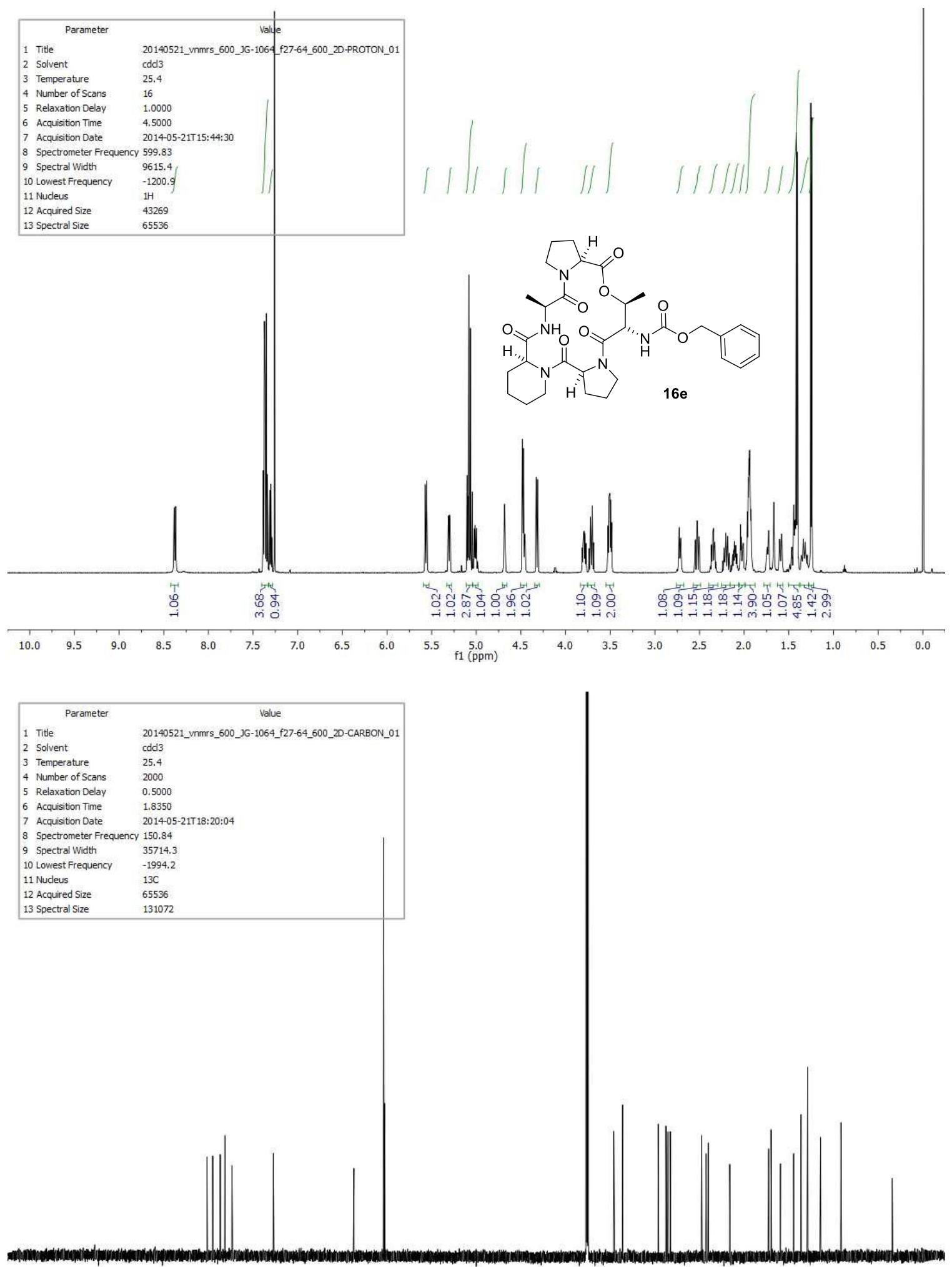

\begin{tabular}{lllllllllllllllllllllllll}
\hline & 220 & 210 & 200 & 190 & 180 & 170 & 160 & 150 & 140 & 130 & 120 & 110 & 100 & 90 & 80 & 70 & 60 & 50 & 40 & 30 & 20 & 10 & 0 & -10
\end{tabular} 

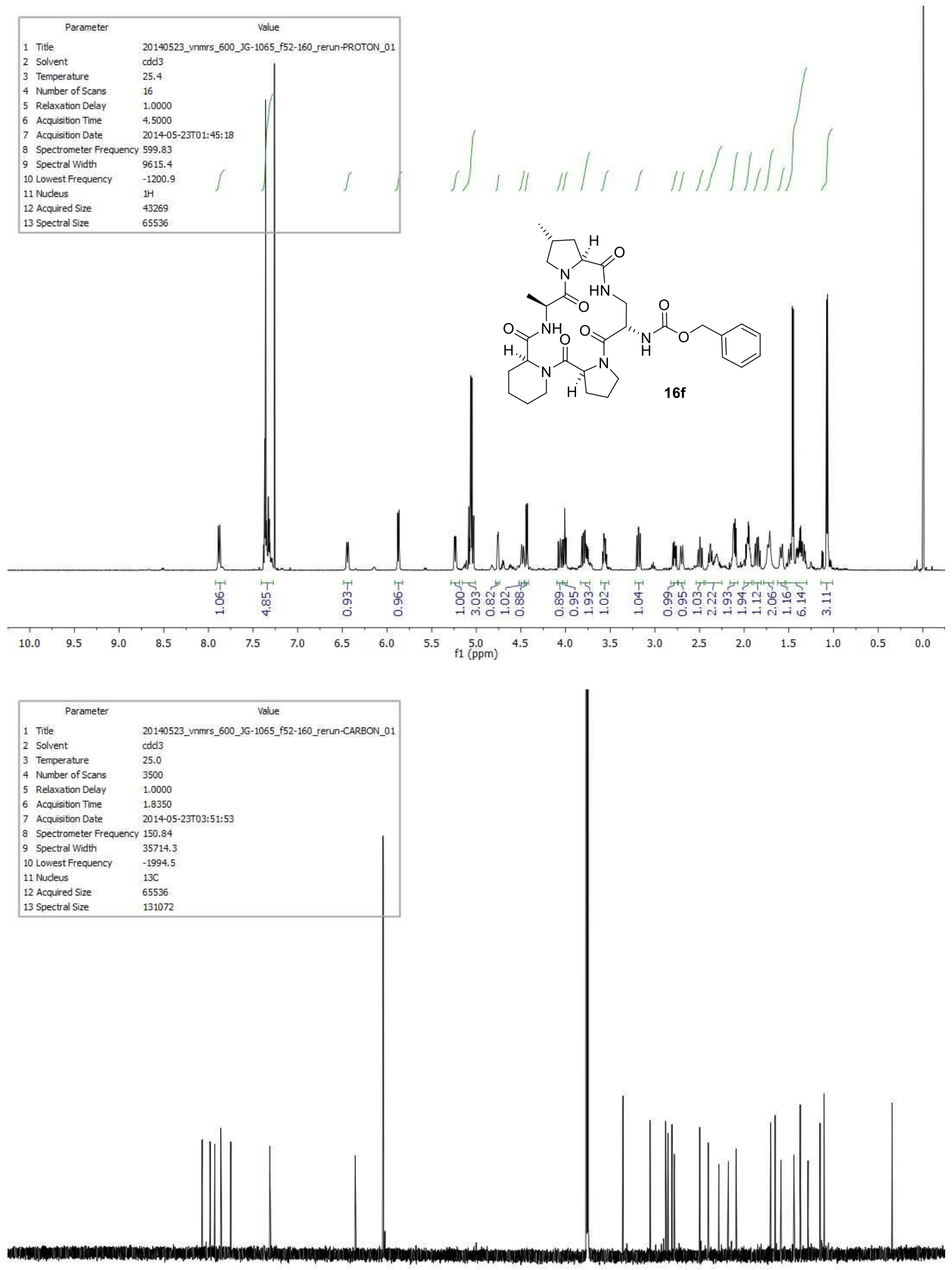

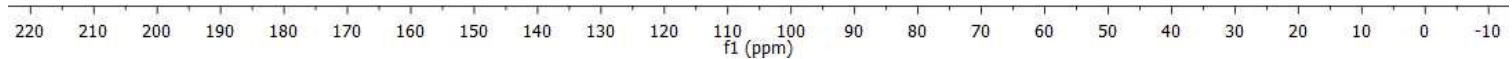




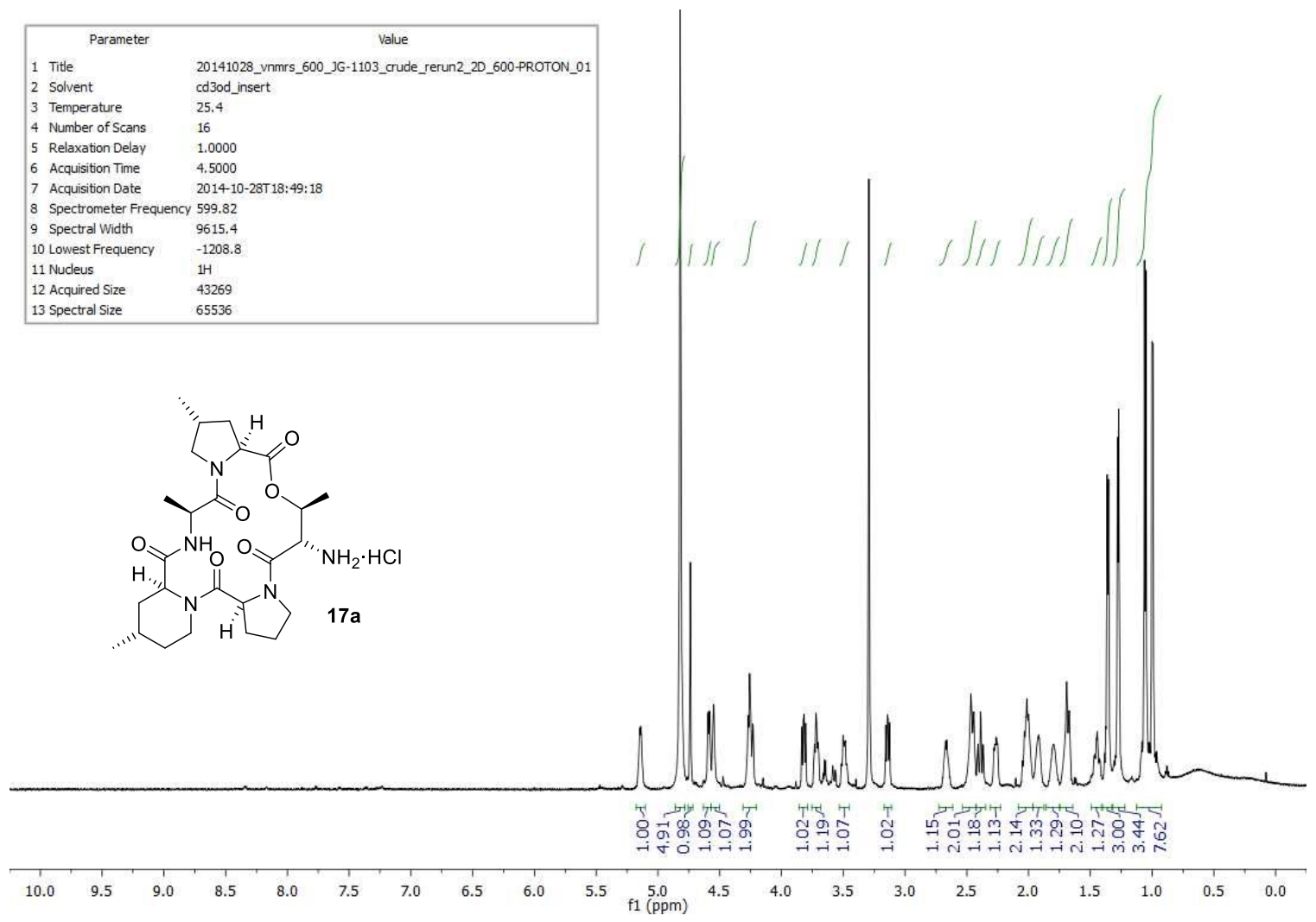

\begin{tabular}{|lll|}
\hline \multicolumn{1}{|c|}{ Parameter } & \multicolumn{1}{c|}{ Value } \\
1 & Tite & 20141028_vnmrs_500_JG-1103_crude_rerun2-CARBON_01 \\
2 & Solvent & cd3od_insert \\
3 & Temperature & 25.0 \\
4 & Number of Scans & 650 \\
5 & Relaxation Delay & 0.2000 \\
6 & Acquisition Time & 2.0000 \\
7 & Acquisition Date & $2014-10-28 T 09: 17: 46$ \\
8 & Spectrometer Frequency & 125.65 \\
9 & Spectral Width & 30487.8 \\
10 & Lowest Frequency & -1856.4 \\
11 & Nudleus & $13 C$ \\
12 Acquired Size & 60976 \\
13 Spectral Size & 131072 \\
\hline
\end{tabular}

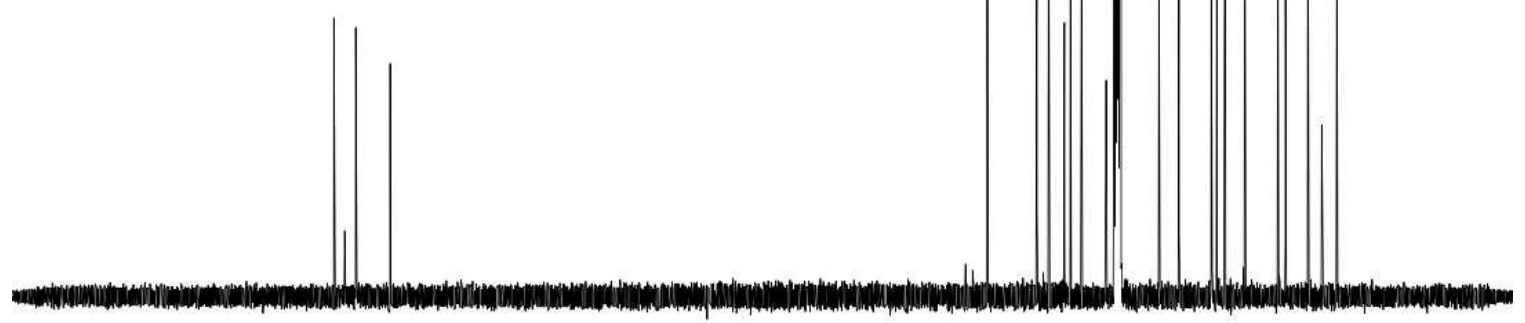

$\begin{array}{llllllllllllllllllllllllll}220 & 210 & 200 & 190 & 180 & 170 & 160 & 150 & 140 & 130 & 120 & \underset{\mathrm{f} 1}{110}(\mathrm{ppm}) & 100 & 90 & 80 & 70 & 60 & 50 & 40 & 30 & 20 & 10 & 0 & -10\end{array}$ 


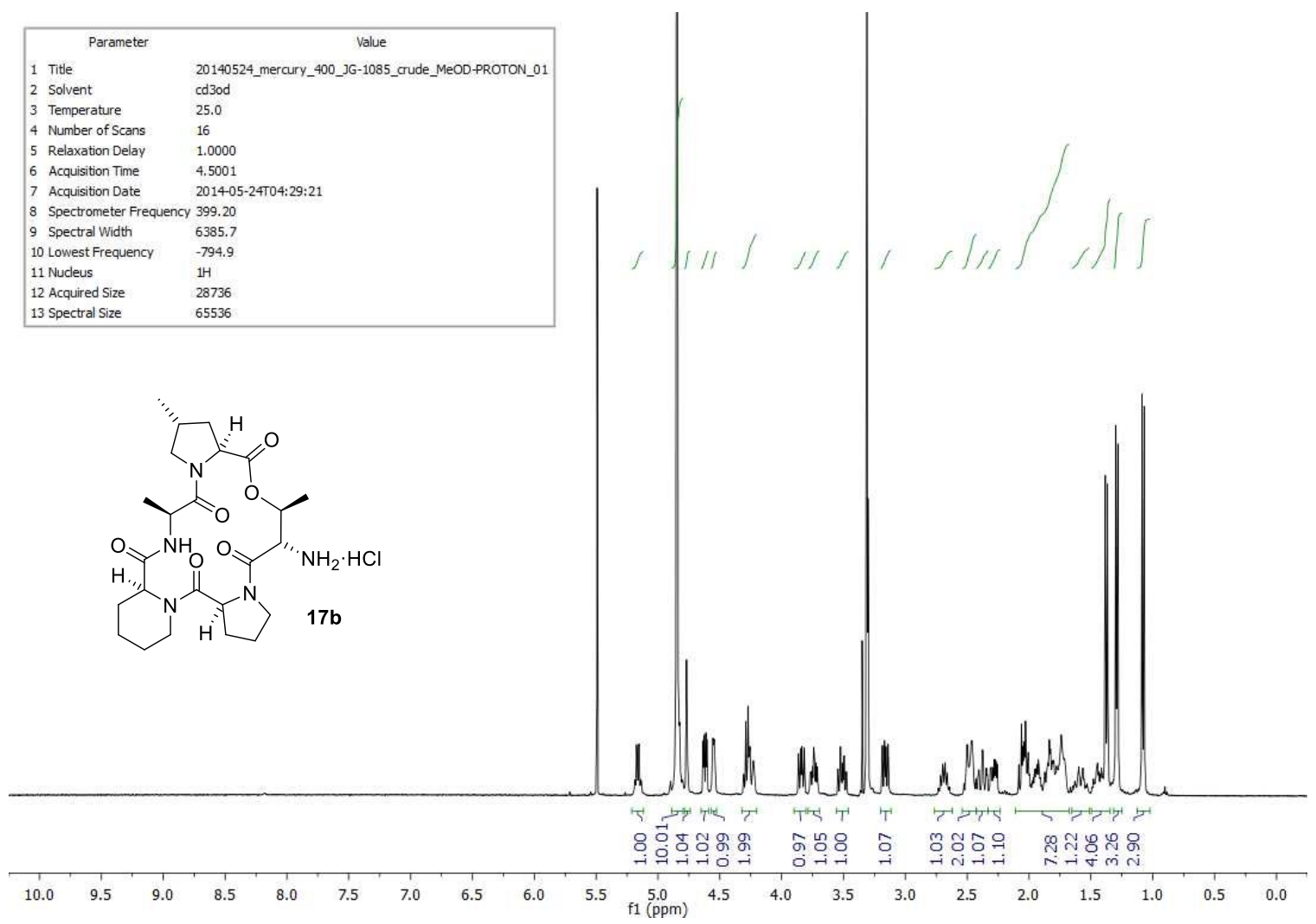

\begin{tabular}{|lll|}
\hline \multicolumn{1}{|c|}{ Parameter } & \multicolumn{1}{c|}{ Value } \\
1 & Title & 20140524 mercury_400_JG-1085_crude_MeOD-CARBON_01 \\
2 & Solvent & cd3od \\
3 & Temperature & 25.0 \\
4 & Number of Scans & 2000 \\
5 & Relaxation Delay & 1.0000 \\
6 & Acquisition Time & 2.0000 \\
7 & Acquisition Date & $2014-05-24104: 59: 16$ \\
8 & Spectrometer Frequency & 100.39 \\
9 & Spectral Width & 25125.6 \\
10 & Lowest Frequency & -1363.0 \\
11 Nucleus & $13 C$ \\
12 Acquired Size & 50251 \\
13 Spectral Size & 131072 \\
\hline
\end{tabular}

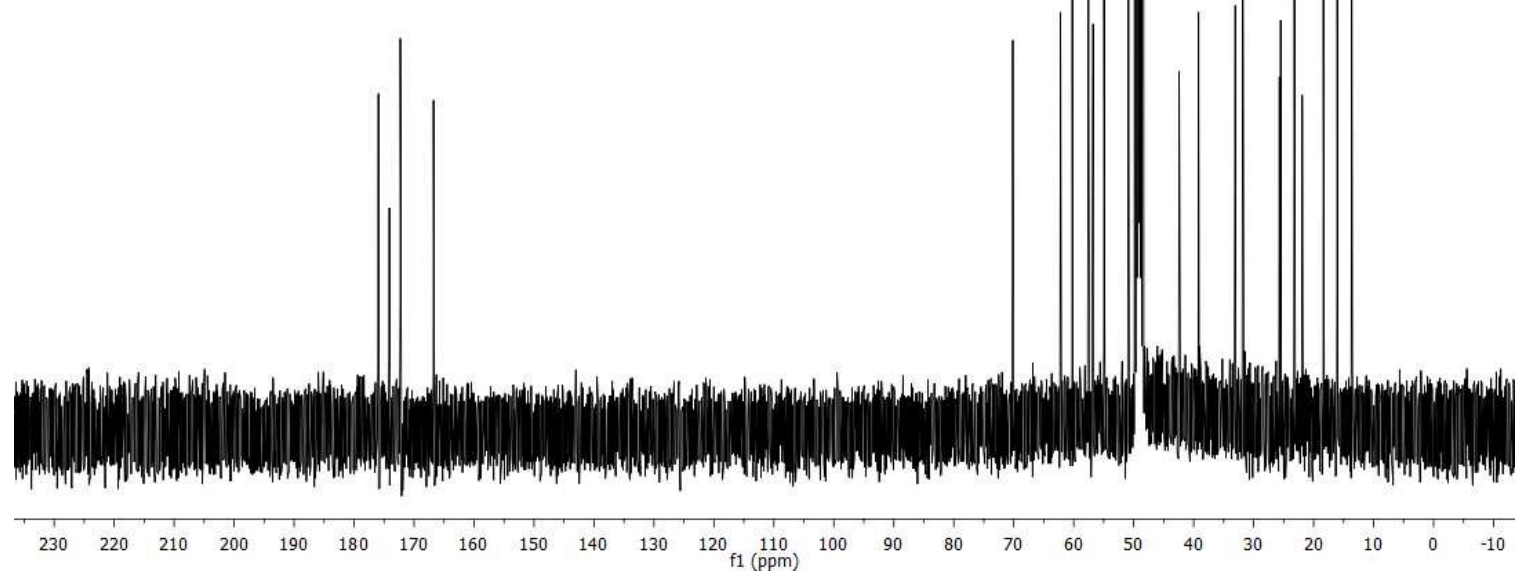




\begin{tabular}{|lll|}
\hline \multicolumn{1}{|c|}{ Parameter } & \multicolumn{1}{c|}{ Value } \\
1 & Title & 20141028_vnmrs_600_JG-1105_crude_rerun2_2D_600-PROTON_01 \\
2 & Solvent & cd3od_insert \\
3 & Temperature & 25.4 \\
4 & Number of Scans & 16 \\
5 & Relaxation Delay & 1.0000 \\
6 & Acquisition Time & 4.5000 \\
7 & Acquisition Date & $2014-10-28 T 20: 26: 29$ \\
8 & Spectrometer Frequency 599.82 \\
9 & Spectral Width & 9615.4 \\
10 & Lowest Frequency & -1198.0 \\
11 & Nucleus & $1 H$ \\
12 & Acquired Size & 43269 \\
13 & Spectral Size & 65536 \\
\hline
\end{tabular}
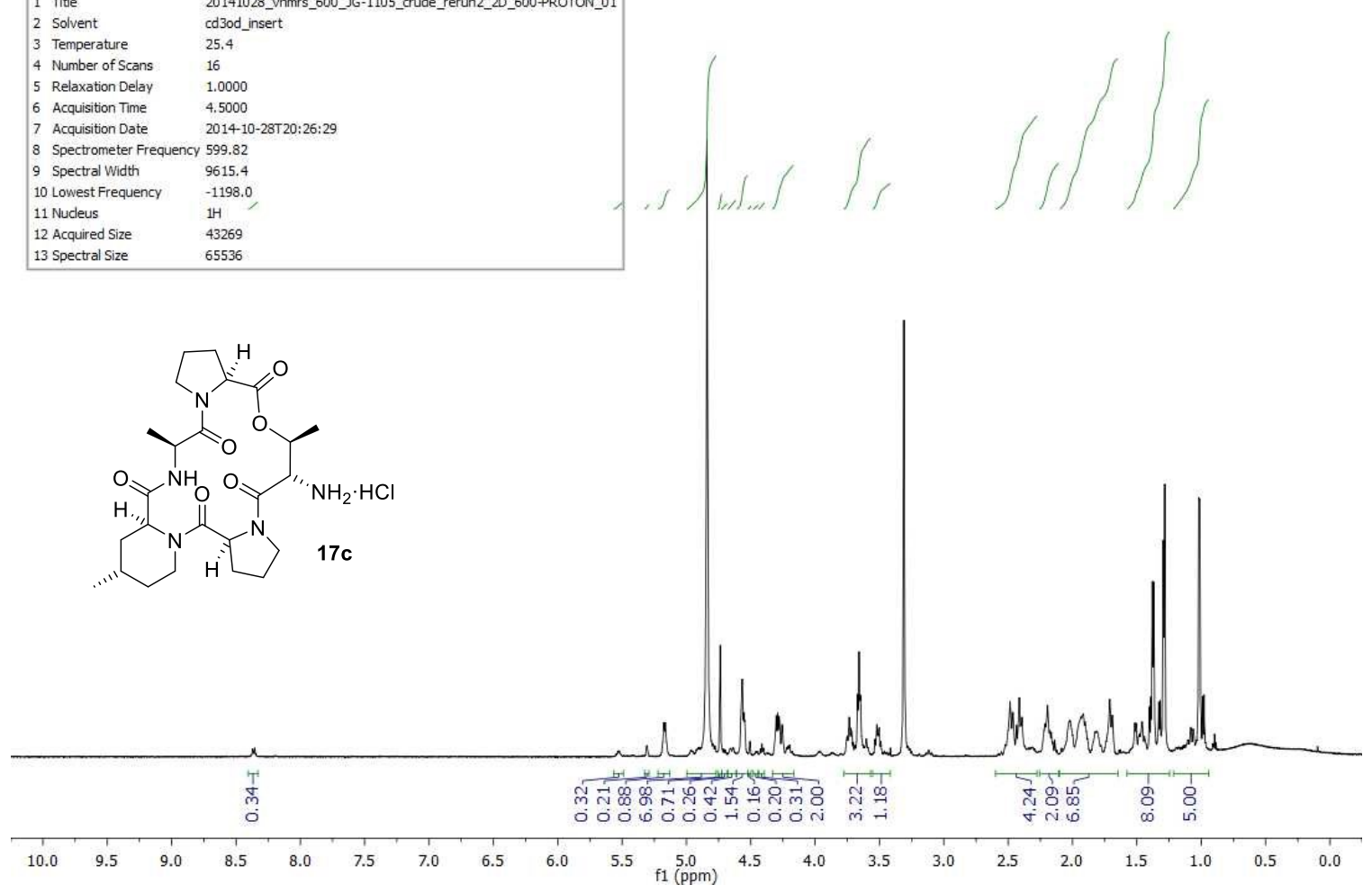

\begin{tabular}{|c|c|}
\hline Parameter & Value \\
\hline 1 Title & 20141028_ynmrs_500_JG-1105_crude_rerun2-CARBON_01 \\
\hline 2 Solvent & cd3od_insert \\
\hline 3 Temperature & 25.0 \\
\hline 4 Number of Scans & 650 \\
\hline 5 Relaxation Delay & 0.2000 \\
\hline 6 Acquisition Time & 2,0000 \\
\hline 7 Acquisition Date & 2014-10-28T 10:21:14 \\
\hline 8 Spectrometer Frequency & 125.65 \\
\hline 9 Spectral Width & 30487.8 \\
\hline 10 Lowest Frequency & -1855.9 \\
\hline 11 Nudeus & $13 \mathrm{C}$ \\
\hline 12 Acquired Size & 60976 \\
\hline 13 Spectral Size & 131072 \\
\hline
\end{tabular}

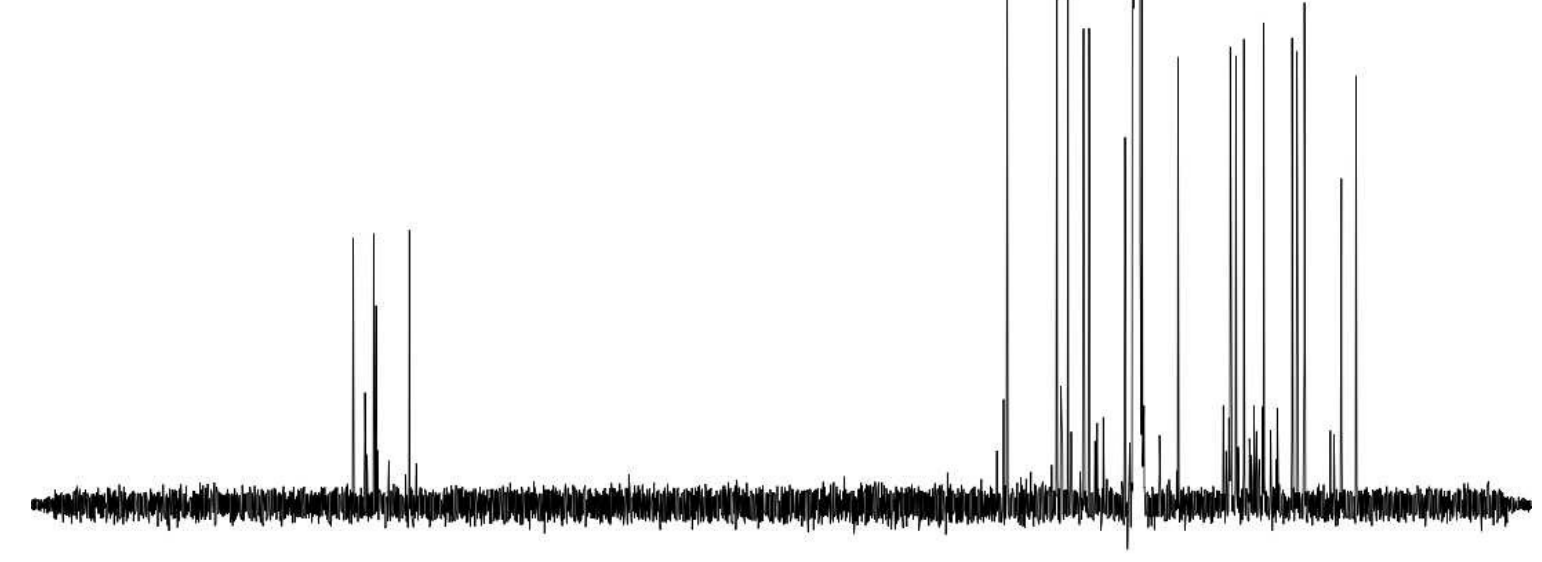

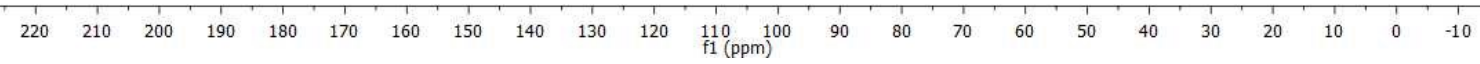




\begin{tabular}{|lll|}
\hline \multicolumn{1}{|c|}{ Parameter } & \multicolumn{1}{c|}{ Value } \\
1 & Title & 20141028_vnmrs_600_JG-1104_crude_rerun2_2D_600-PROTON_01 \\
2 & Solvent & cd3od_insert \\
3 & Temperature & 25.4 \\
4 & Number of Scans & 16 \\
5 & Relaxation Delay & 1.0000 \\
6 & Acquisition Time & 4.5000 \\
7 & Acquisition Date & $2014-10-28 T 17: 12: 17$ \\
8 & Spectrometer Frequency 599.82 \\
9 & Spectral Width & 9615.4 \\
10 & Lowest Frequency & -1198.6 \\
11 & Nucleus & $1 H$ \\
12 & Acquired Size & 43269 \\
13 & Spectral Size & 65536 \\
\hline
\end{tabular}
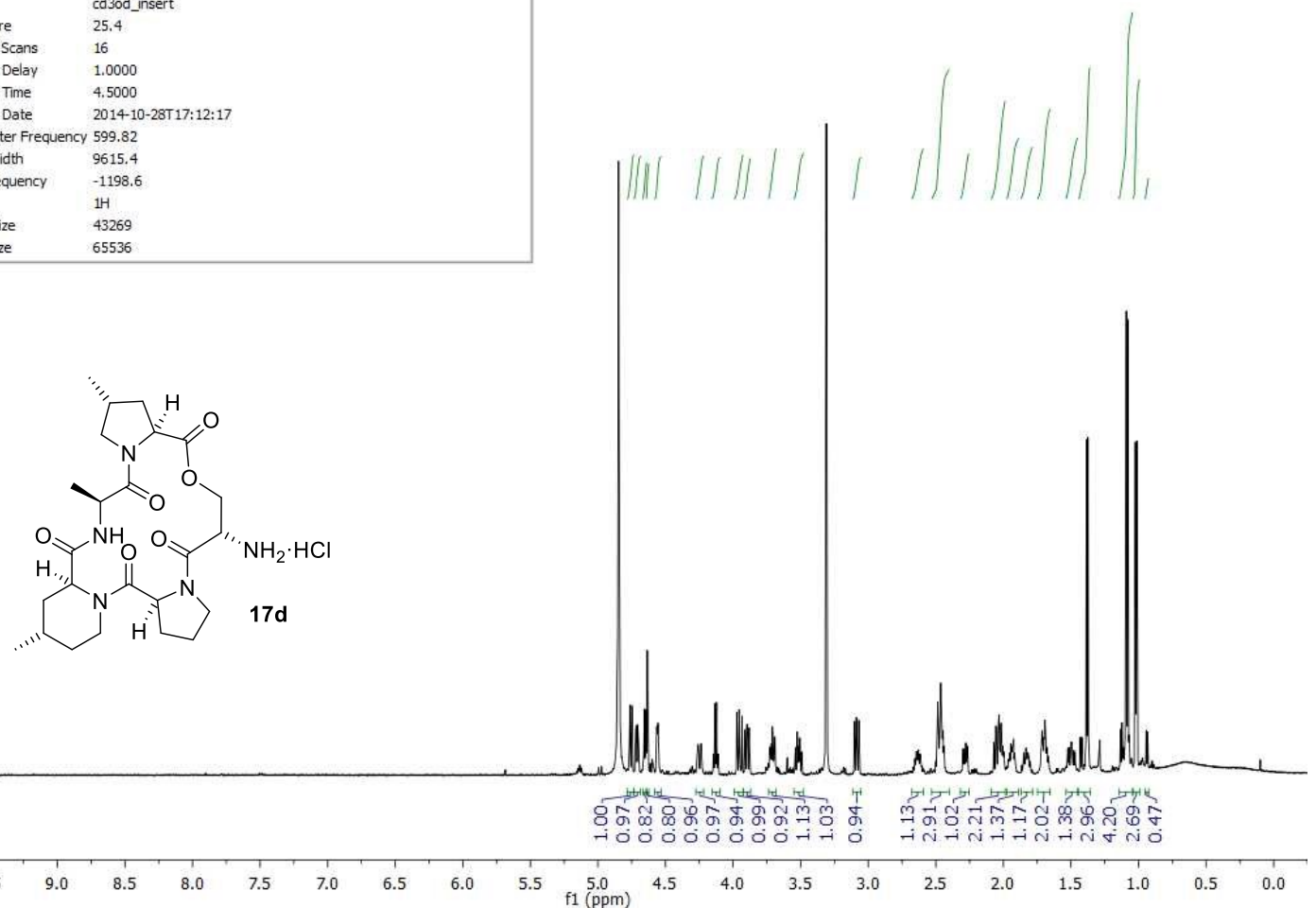

\begin{tabular}{|lll|}
\hline \multicolumn{1}{|c|}{ Parameter } & \multicolumn{1}{c|}{ Value } \\
1 & Tite & 20141028_ynmrs_500_JG-1104_crude_rerun2-CARBON_01 \\
2 & Solvent & cd3od_insert \\
3 & Temperature & 25.0 \\
4 & Number of Scans & 650 \\
5 & Relaxation Delay & 0.2000 \\
6 & Acquisition Time & 2.0000 \\
7 & Acquisition Date & $2014-10-28 T 09: 49: 34$ \\
8 & Spectrometer Frequency & 125.65 \\
9 & Spectral Width & 30487.8 \\
10 & Lowest Frequency & -1854.4 \\
11 Nudleus & $13 C$ \\
12 Acquired Size & 60976 \\
13 Spectral Size & 131072 \\
\hline
\end{tabular}

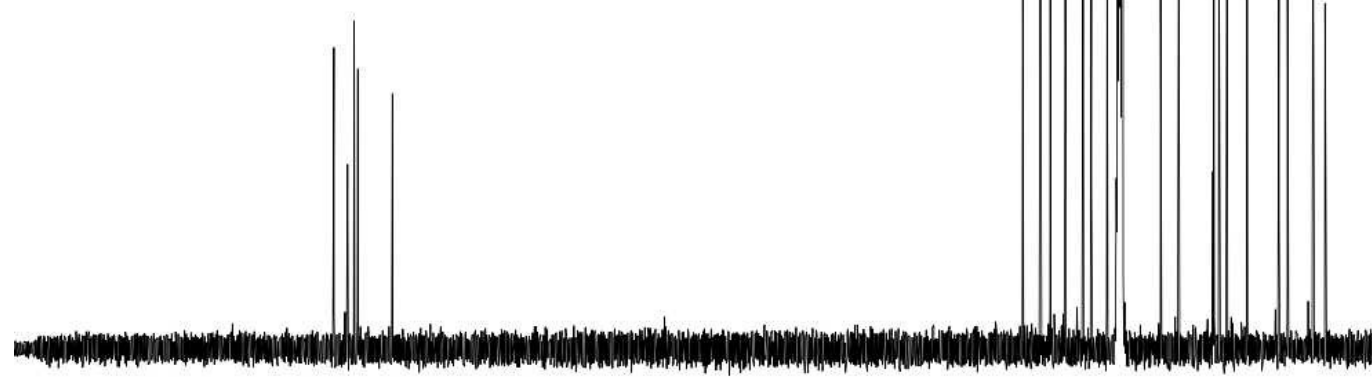

$\begin{array}{llllllllllllllllllllllllll}220 & 210 & 200 & 190 & 180 & 170 & 160 & 150 & 140 & 130 & 120 & \underset{\mathrm{f} 1}{11}(\mathrm{ppm}) & 100 & 90 & 80 & 70 & 60 & 50 & 40 & 30 & 20 & 10 & 0 & -10\end{array}$ 


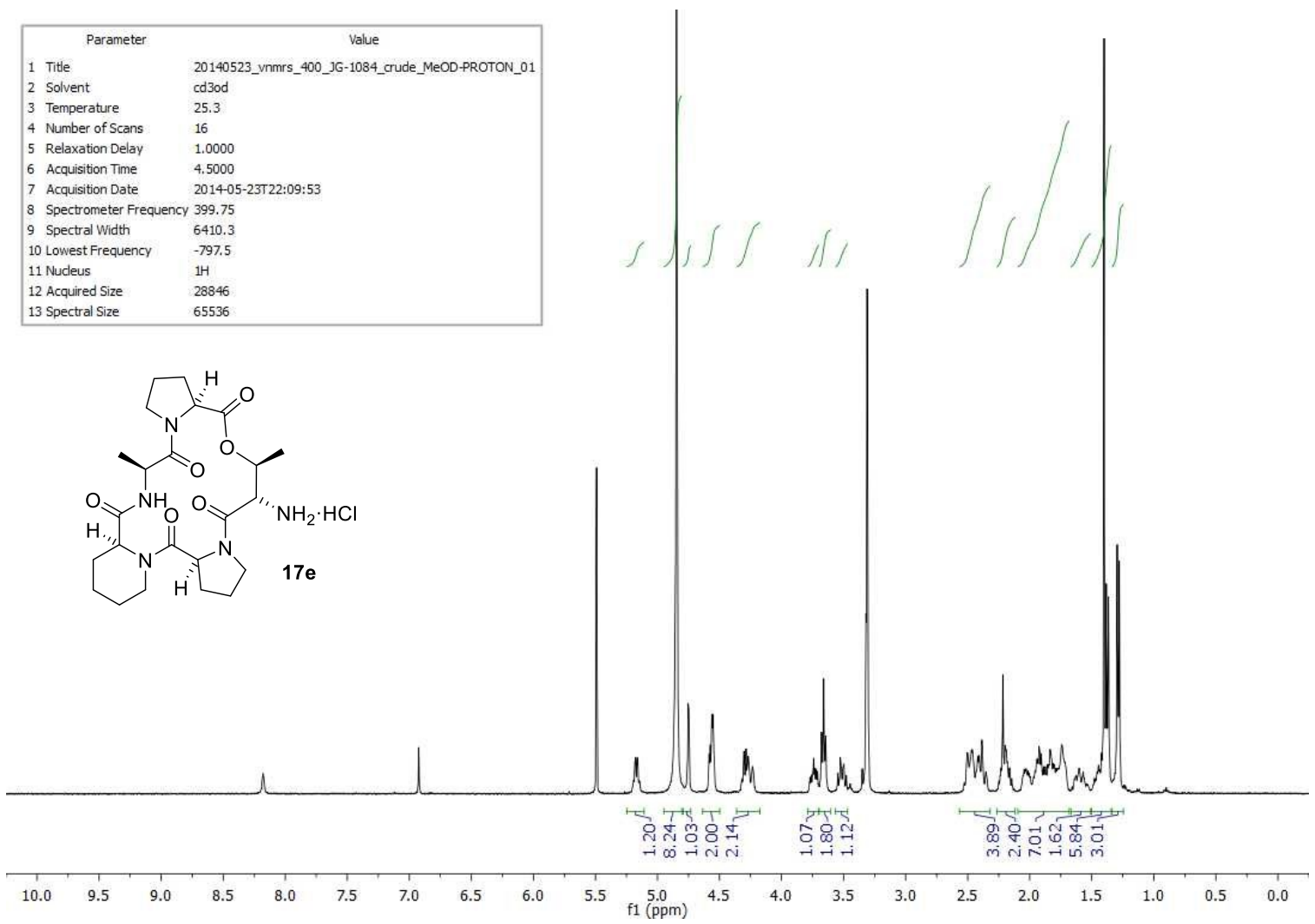

\begin{tabular}{|lll|}
\hline \multicolumn{1}{|c|}{ Parameter } & \multicolumn{1}{c|}{ Value } \\
1 & Title & 20140523 ynmrs_400_JG-1084_crude_MeOD-CARBON_01 \\
2 & Solvent & cd30d \\
3 & Temperature & 25.3 \\
4 & Number of Scans & 2000 \\
5 & Relaxation Delay & 1.0000 \\
6 & Acquisition Time & 1.3631 \\
7 & Acquisition Date & $2014-05-23 T 22: 19: 55$ \\
8 & Spectrometer Frequency & 100.53 \\
9 & Spectral Width & 24038.5 \\
10 & Lowest Frequency & -1305.0 \\
11 & Nucleus & $13 \mathrm{C}$ \\
12 & Acquired Size & 32768 \\
13 & Spectral Size & 65536 \\
\hline
\end{tabular}

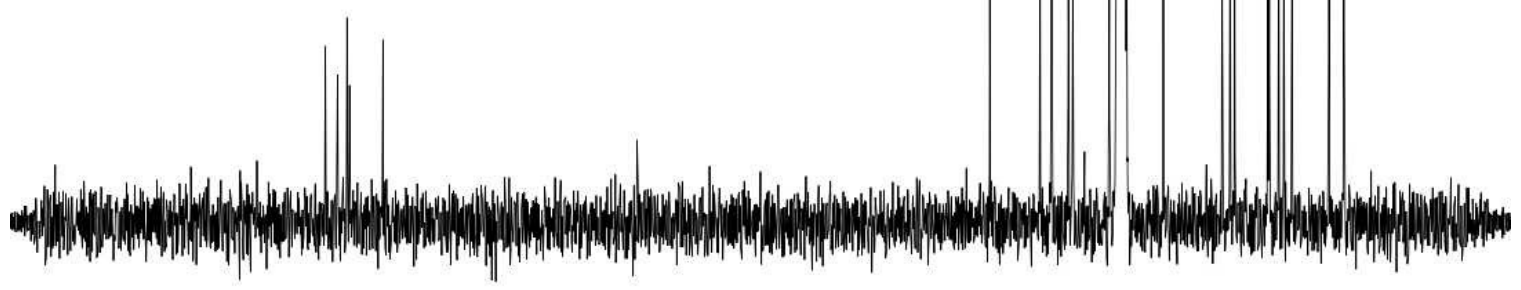

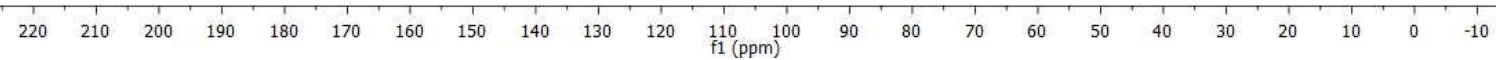




\begin{tabular}{|lll|}
\hline \multicolumn{1}{|c|}{ Parameter } & \multicolumn{1}{c|}{ Value } \\
1 & Title & $20140526 \_$ynmrs_500_JG-1088_crude_DMSO_500-PROTON_01 \\
2 & Solvent & dmso \\
3 & Temperature & 25.0 \\
4 & Number of Scans & 16 \\
5 & Relaxation Delay & 1.0000 \\
6 & Acquisition Time & 4.5000 \\
7 & Acquisition Date & $201405-26$ T04:42:58 \\
8 & Spectrometer Frequency & 499.67 \\
9 & Spectral Width & 8012.8 \\
10 & Lowest Frequency & -1003.0 \\
11 & Nucleus & $1 H$ \\
12 & Acquired Size & 36058 \\
13 & Spectral Size & 65536 \\
\hline
\end{tabular}

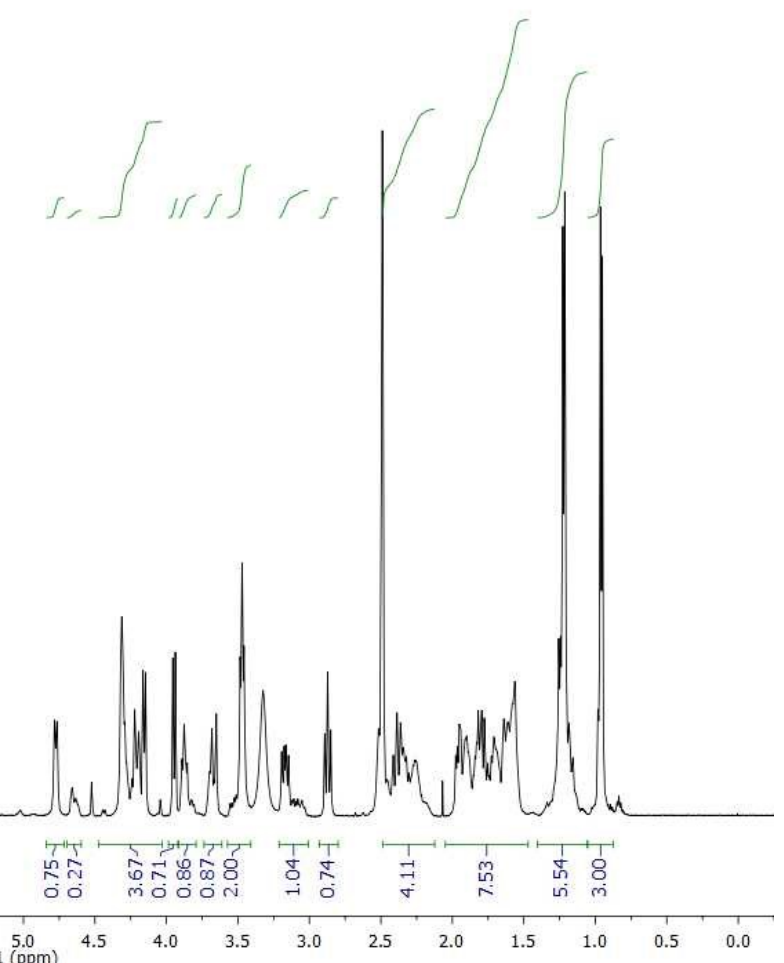

\begin{tabular}{|c|c|}
\hline Parameter & Value \\
\hline 1 Title & 20140526_ynmrs_500_JG-1088_crude_DMSO_500-CARBON_01 \\
\hline 2 Solvent & dmso \\
\hline 3 Temperature & 25.0 \\
\hline 4 Number of Scans & 2000 \\
\hline 5 Relaxation Delay & 1.0000 \\
\hline 6 Acquisition Time & 2.0000 \\
\hline 7 Acquisition Date & 2014-05-26T04:44:52 \\
\hline 8 Spectrometer Frequency & 125.65 \\
\hline 9 Spectral Width & 30487.8 \\
\hline 10 Lowest Frequency & -2051.7 \\
\hline 11 Nucleus & $13 \mathrm{C}$ \\
\hline 12 Acquired Size & 60976 \\
\hline 13 Spectral Size & 131072 \\
\hline
\end{tabular}

$\begin{array}{llllllllllll}220 & 210 & 200 & 190 & 180 & 170 & 160 & 150 & 140 & 130 & 120 & 110 \\ \mathrm{f} 1(\mathrm{ppm}) & 100\end{array}$

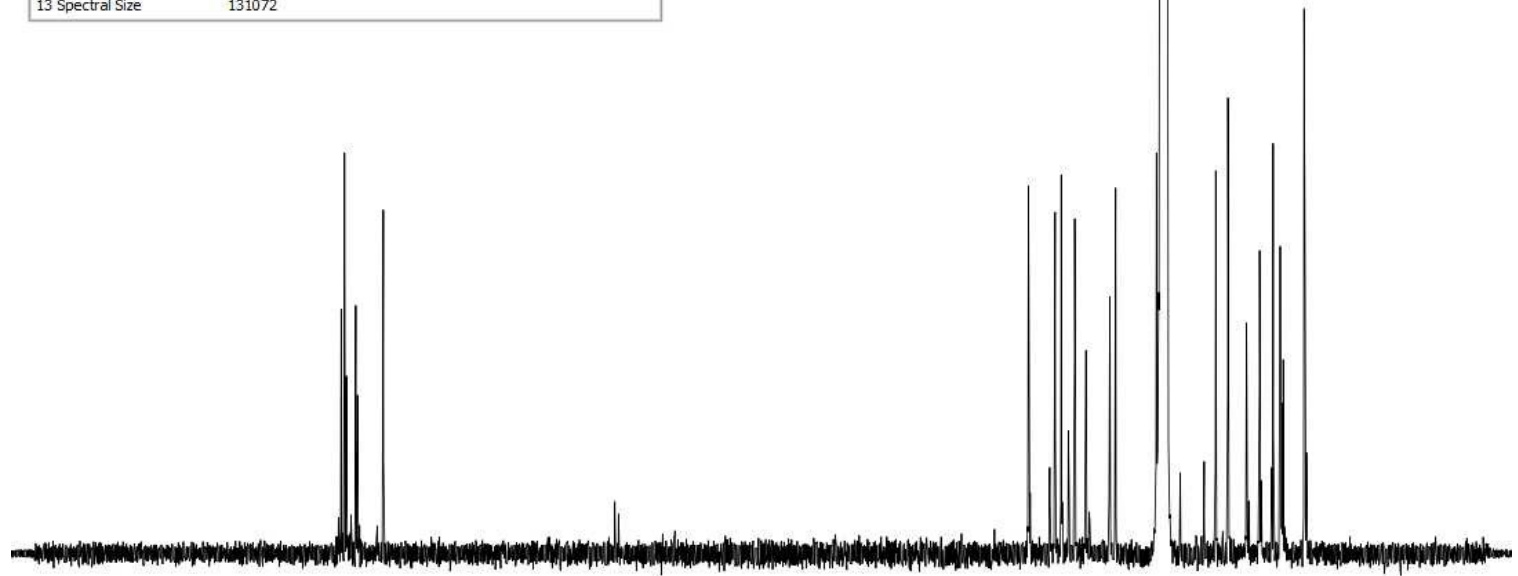



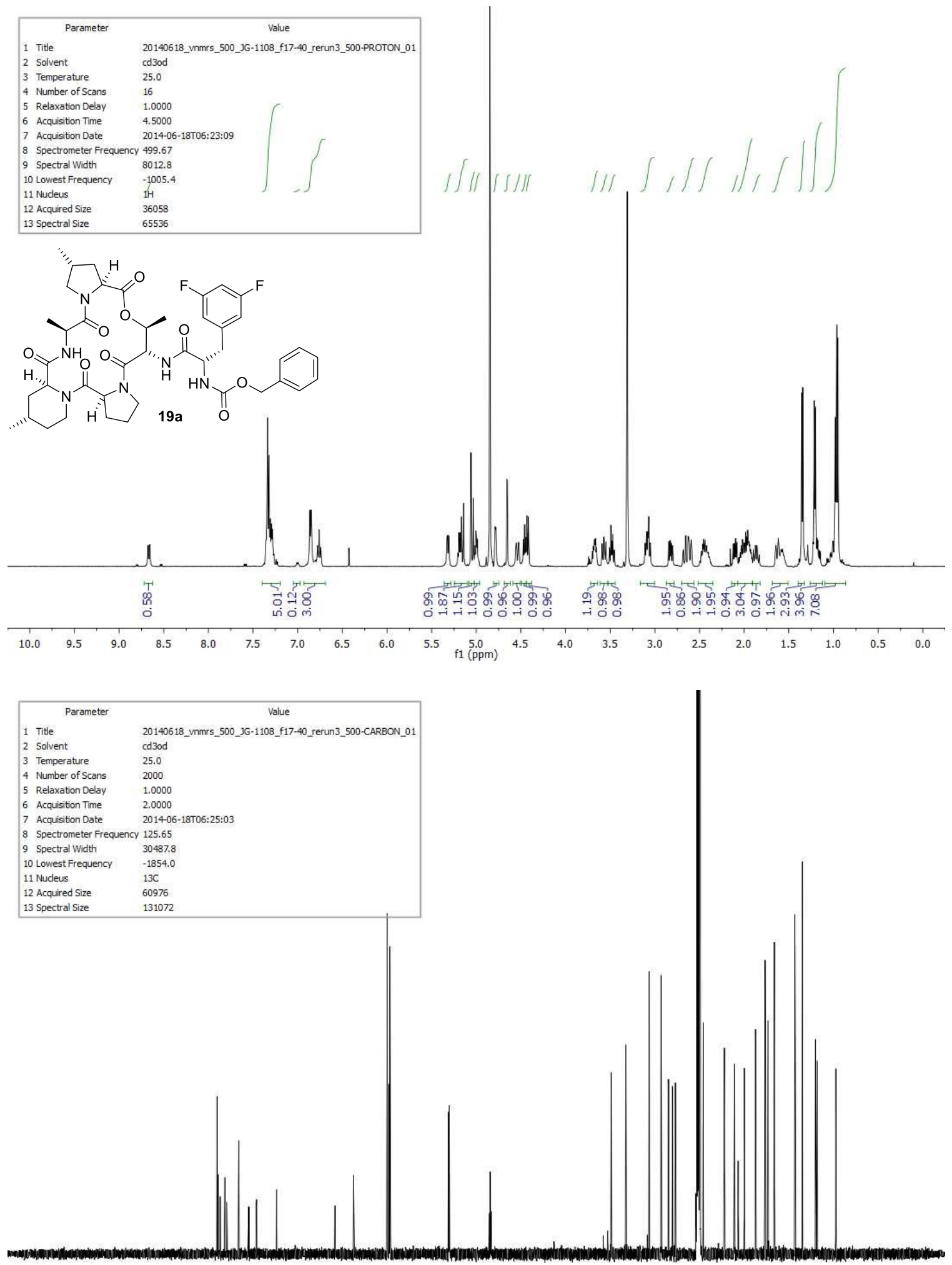

$\begin{array}{llllllllllllllllllllllllll}220 & 210 & 200 & 190 & 180 & 170 & 160 & 150 & 140 & 130 & 120 & \underset{\mathrm{f} 1}{11}(\mathrm{ppm}) & 100 & 90 & 80 & 70 & 60 & 50 & 40 & 30 & 20 & 10 & 0 & -10\end{array}$ 

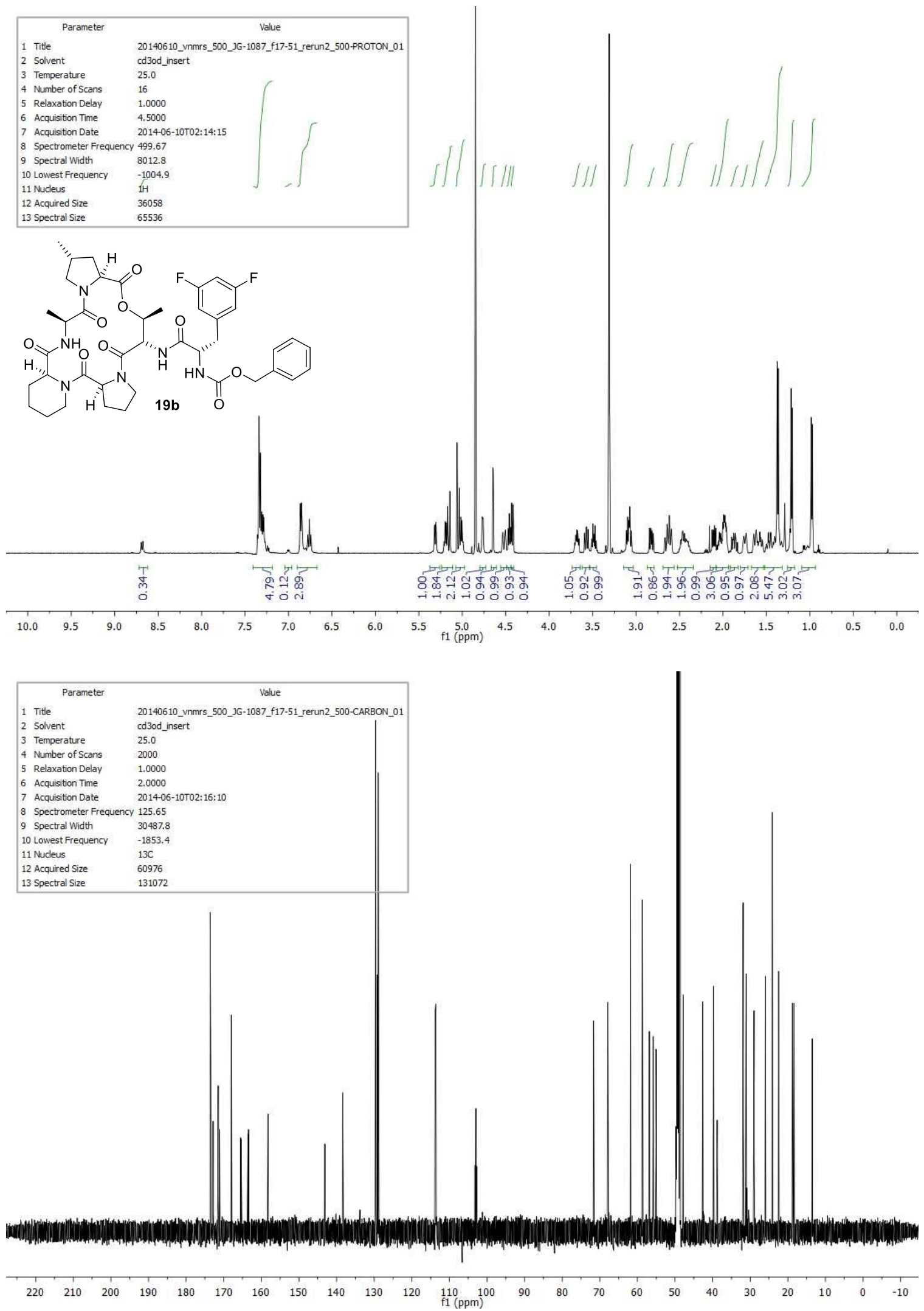

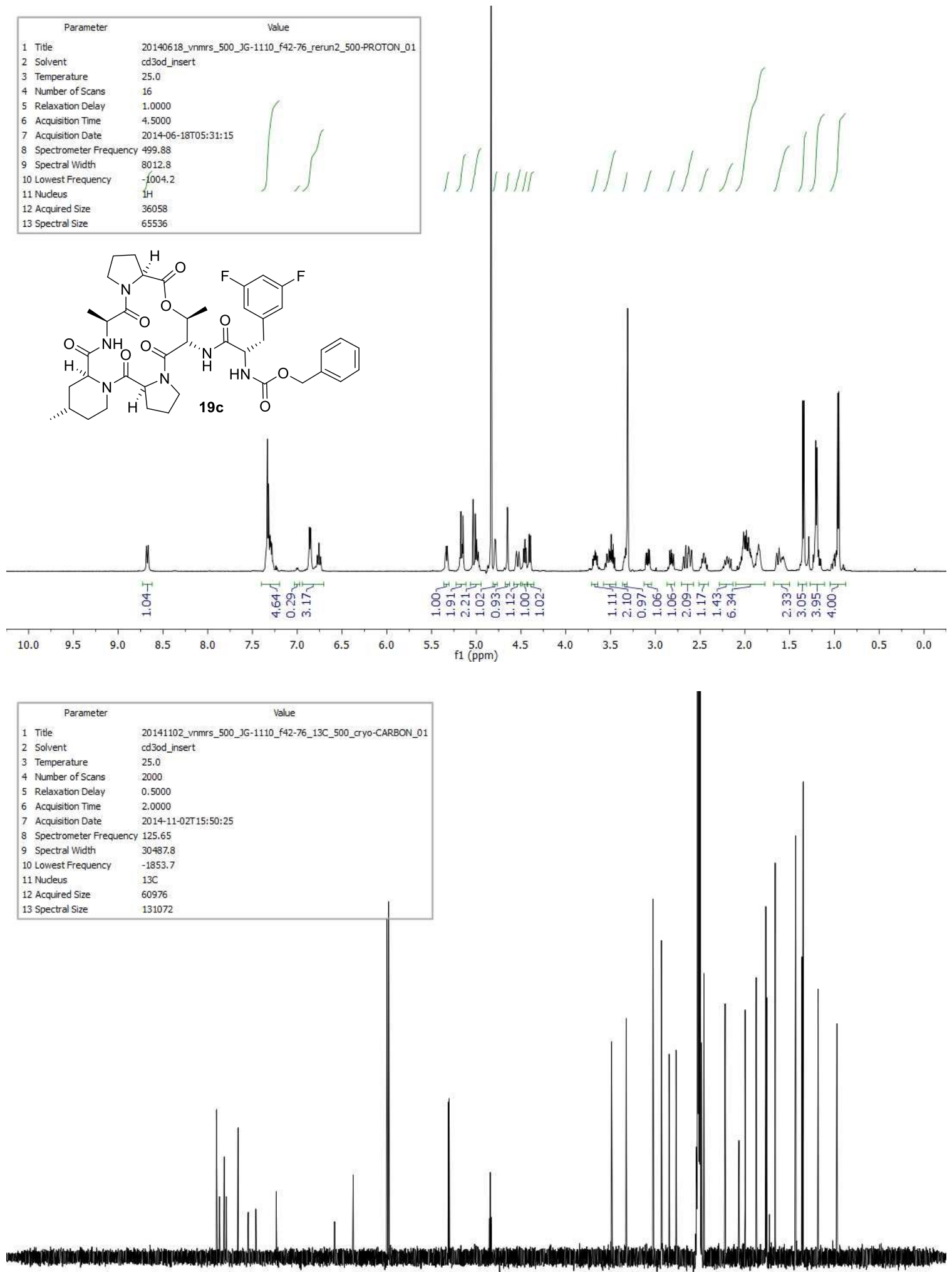

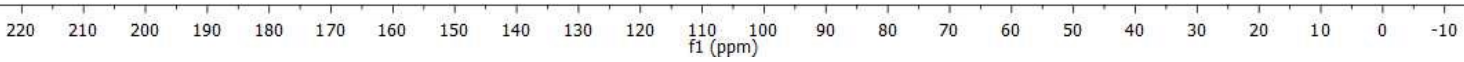



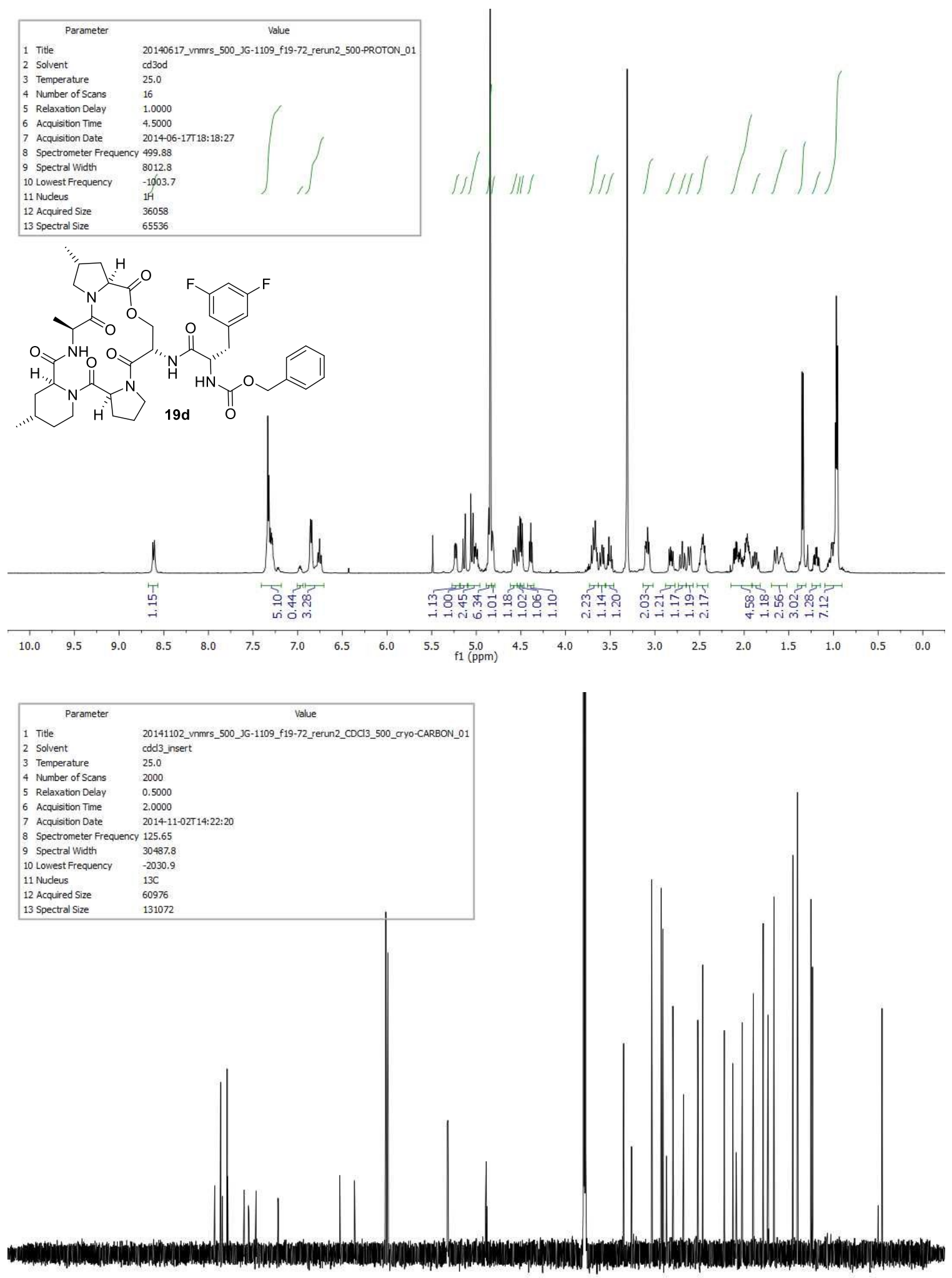

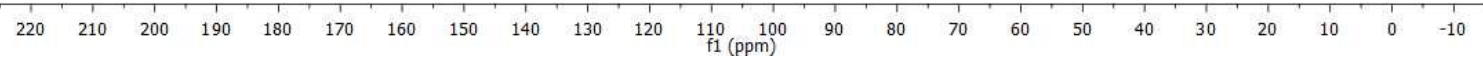




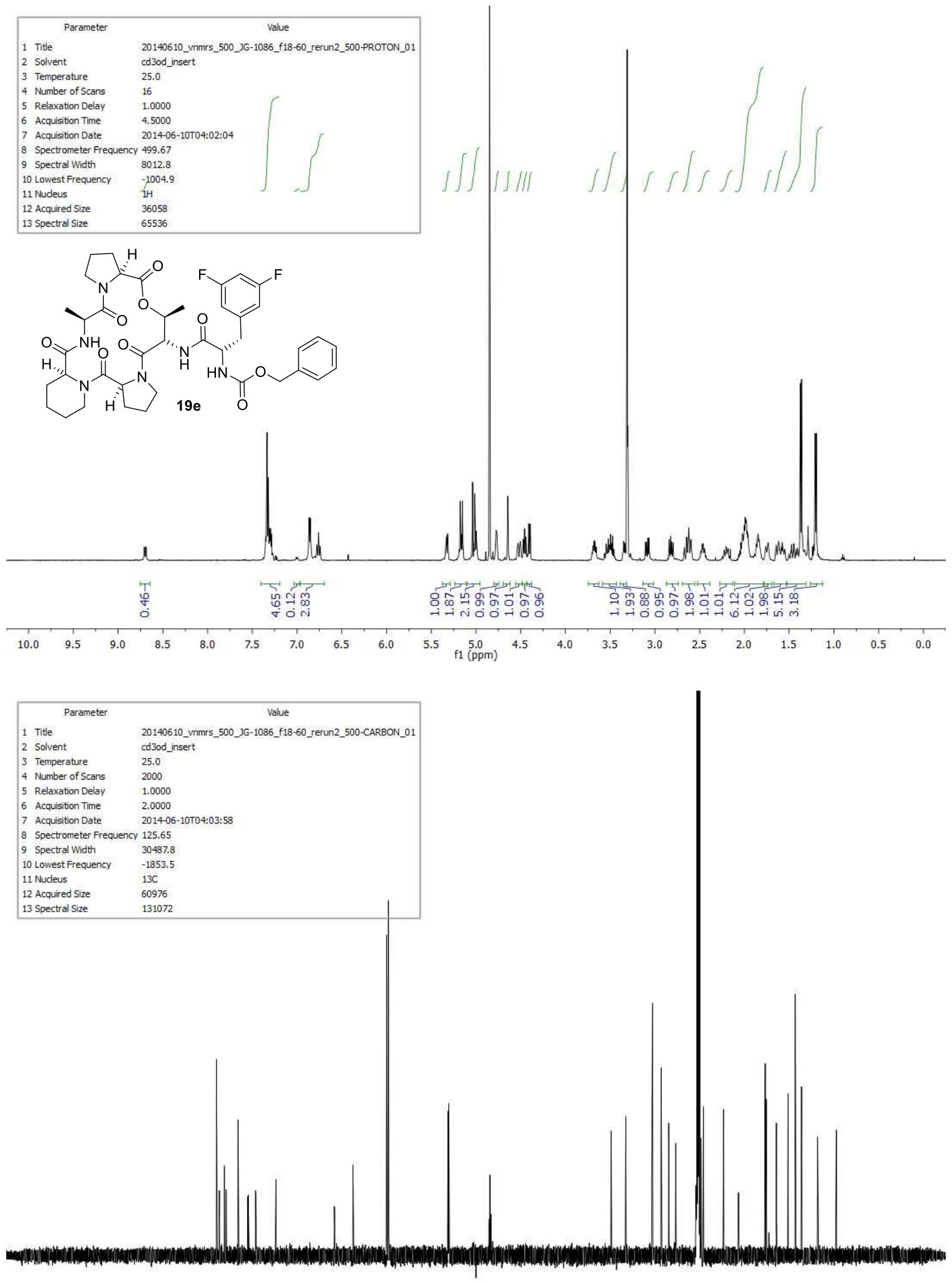

$\begin{array}{llllllllllllllllllllllll}220 & 210 & 200 & 190 & 180 & 170 & 160 & 150 & 140 & 130 & 120 & 110 & 100 & 90 & 80 & 70 & 60 & 50 & 40 & 30 & 20 & 10 & 0 & -10\end{array}$ 


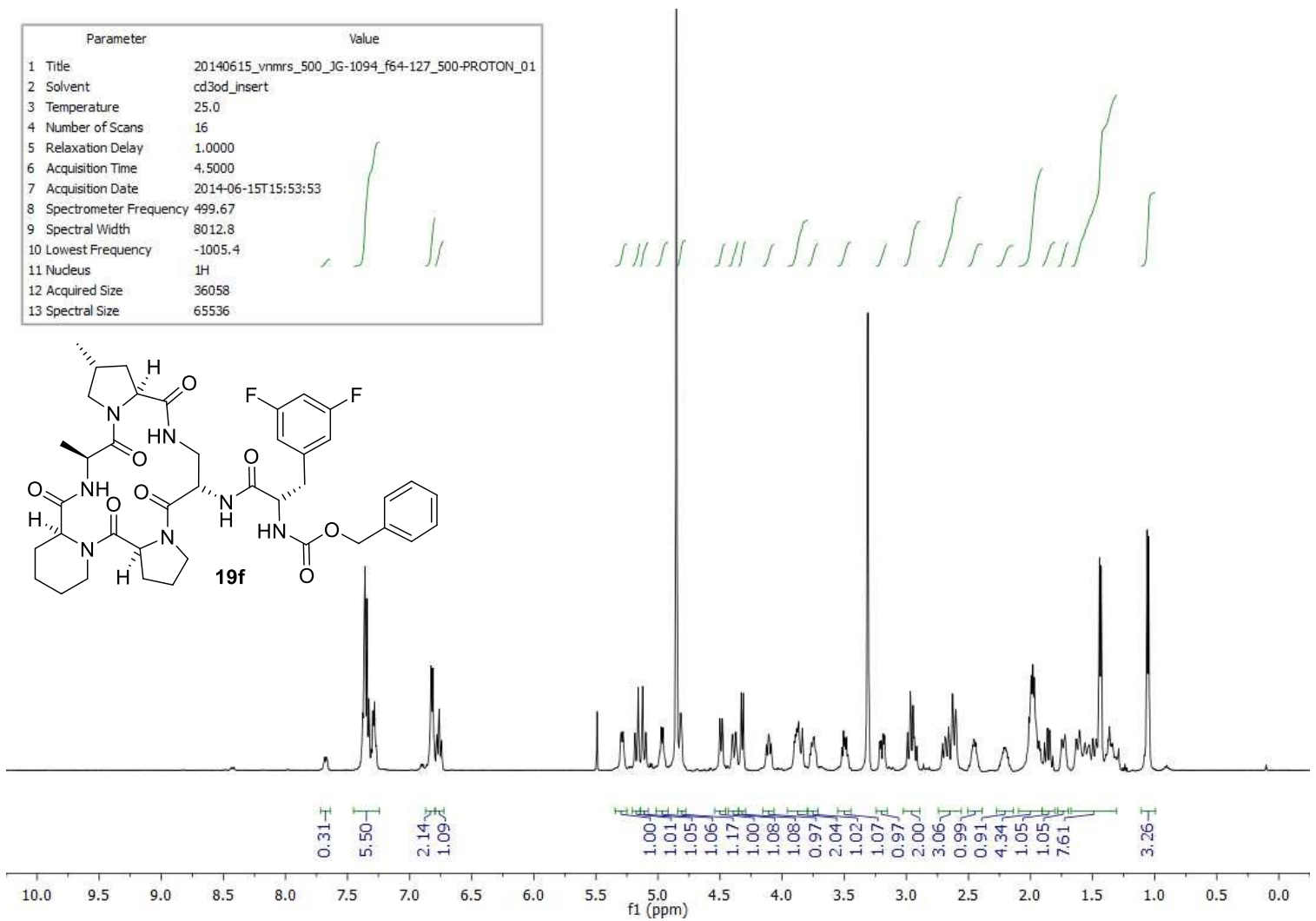

\begin{tabular}{|lll|}
\hline \multicolumn{1}{|c|}{ Parameter } \\
1 & Title & 20140615_ynmrs_500_JG-1094_f64-127_500-CARBON_01 \\
2 & Solvent & cd3od_insert \\
3 & Temperature & 25.0 \\
4 & Number of Scans & 2000 \\
5 & Relaxation Delay & 1.0000 \\
6 & Acquisition Time & 2.0000 \\
7 & Acquisition Date & $2014.06-1515: 55: 50$ \\
8 & Spectrometer Frequency 125.65 \\
9 & Spectral Width & 30487.8 \\
10 & Lowest Frequency & -1853.4 \\
11 & Nucleus & $13 C$ \\
12 & Acquired Size & 60976 \\
13 & Spectral Size & 131072 \\
\hline
\end{tabular}
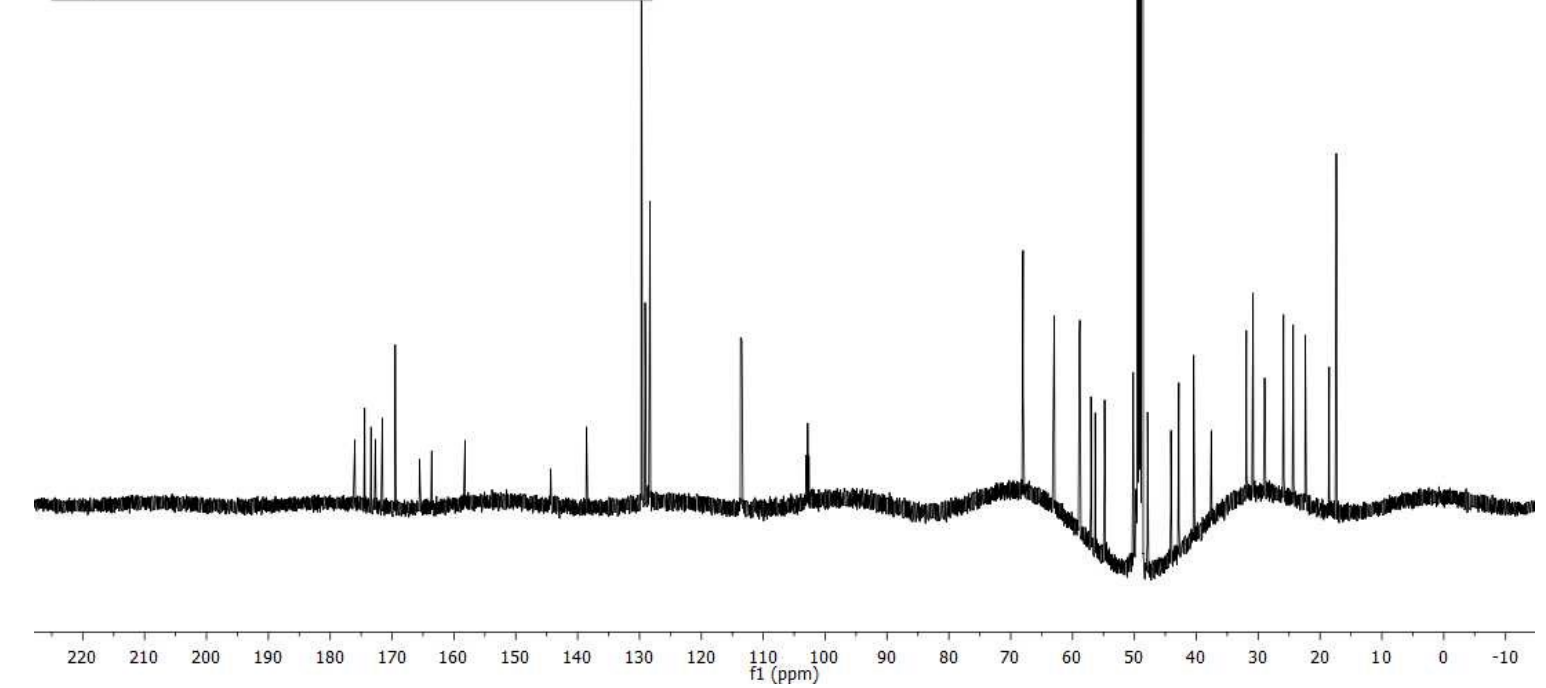

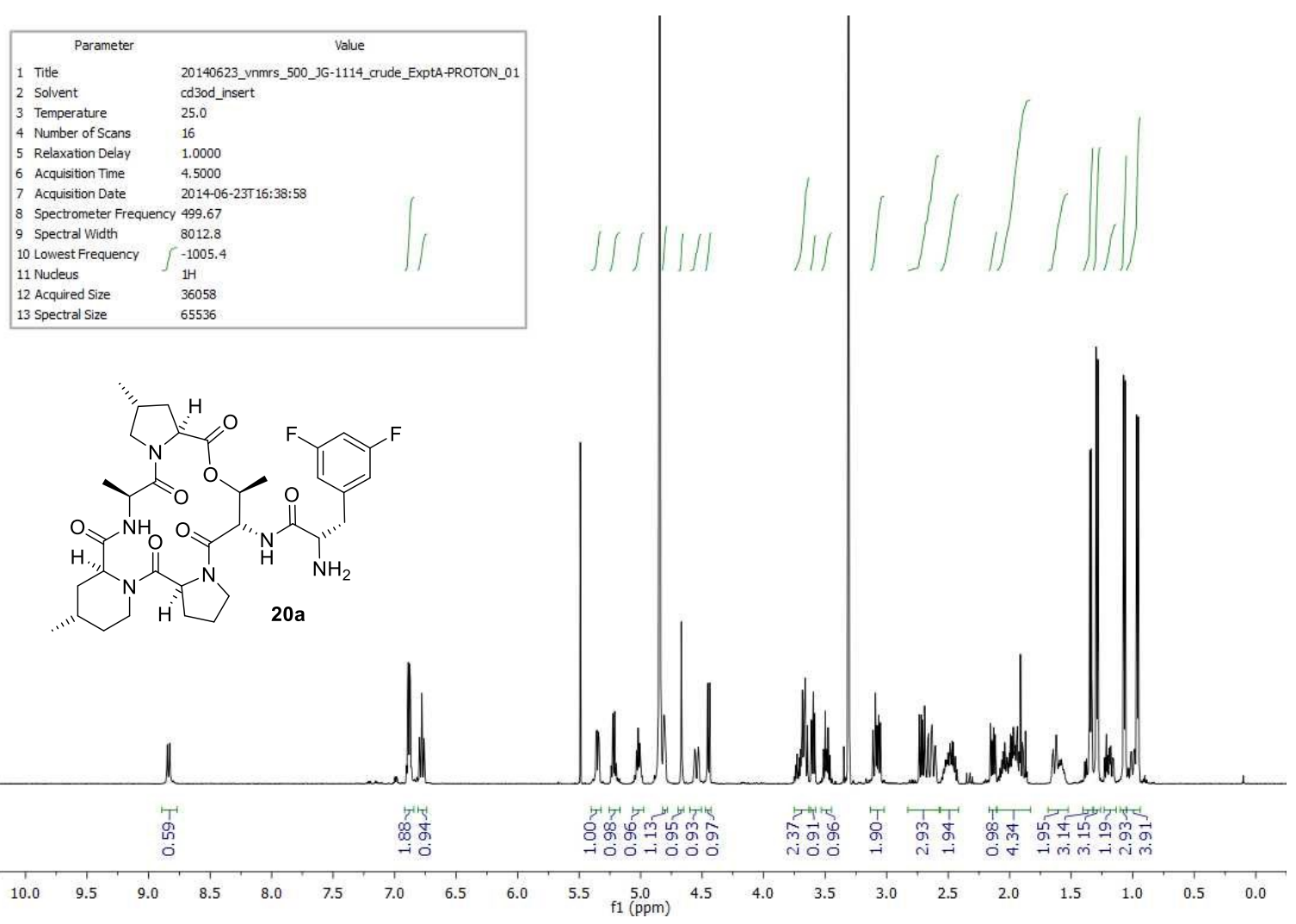

\begin{tabular}{|c|c|}
\hline Parameter & Value \\
\hline 1 Title & 20140625_ynmrs_500_JG-1114_crude_ExptA_2-CARBON_01 \\
\hline 2 Solvent & cd3od_insert \\
\hline 3 Temperature & 25.0 \\
\hline 4 Number of Scans & 2000 \\
\hline 5 Relaxation Delay & 0.2000 \\
\hline 6 Acquisition Time & 2,0000 \\
\hline 7 Acquisition Date & $2014-06-24 T 23: 48: 57$ \\
\hline 8 Spectrometer Frequency & 125.65 \\
\hline 9 Spectral Width & 30487.8 \\
\hline 10 Lowest Frequency & -1853.8 \\
\hline 11 Nucleus & $13 \mathrm{C}$ \\
\hline 12 Acquired Size & 60976 \\
\hline 13 Spectral Size & 131072 \\
\hline
\end{tabular}

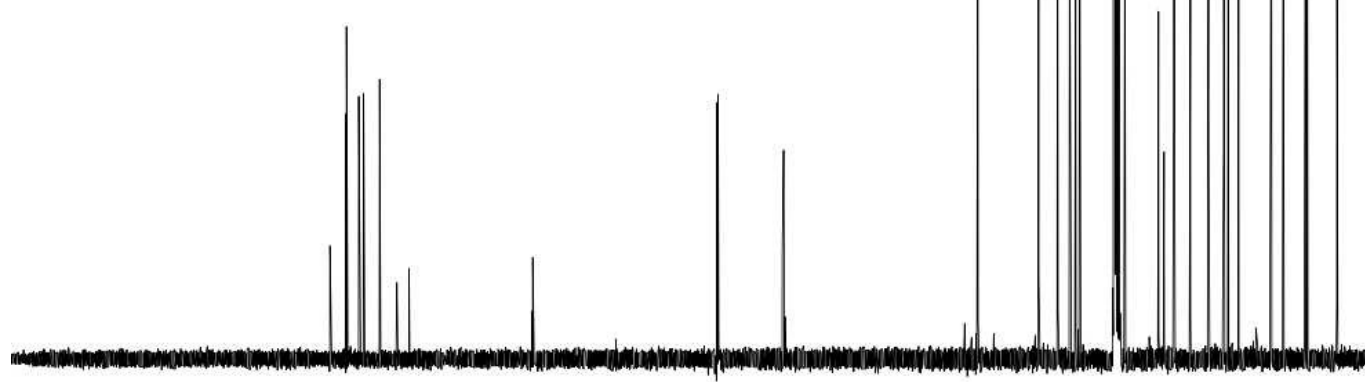

$\begin{array}{llllllllllllllllllllllllll}220 & 210 & 200 & 190 & 180 & 170 & 160 & 150 & 140 & 130 & 120 & \underset{\mathrm{f} 1}{11}(\mathrm{ppm}) & 100 & 90 & 80 & 70 & 60 & 50 & 40 & 30 & 20 & 10 & 0 & -10\end{array}$ 


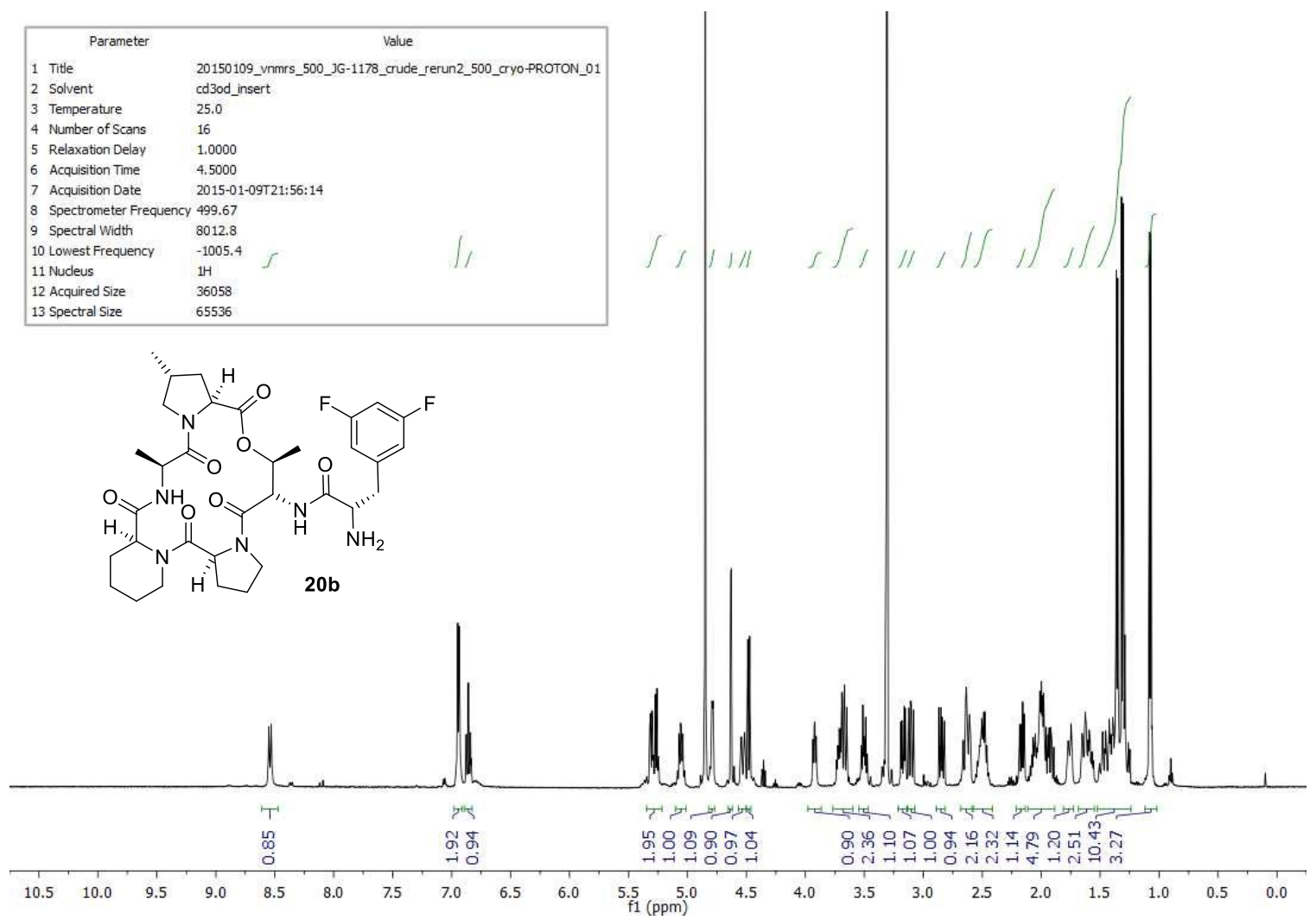

\begin{tabular}{|lll|}
\hline \multicolumn{1}{|c|}{ Parameter } & \multicolumn{1}{c|}{ Value } \\
1 & Title & 20150111 vnnms_500_JG-1178_crude_rerun_13C-CARBON_01 \\
2 & Solvent & cd3od_insert \\
3 & Temperature & 25.0 \\
4 & Number of SCans & 3000 \\
5 & Relaxation Delay & 0.5000 \\
6 & Acquisition Time & 2.0000 \\
7 & Acquisition Date & $2015-01-11 T 17: 06: 34$ \\
8 & Spectrometer Frequency 125.65 \\
9 & Spectral Width & 30487.8 \\
10 & Lowest Frequency & -1853.9 \\
11 & Nucleus & $13 \mathrm{C}$ \\
12 Acquired Size & 60976 \\
13 Spectral Size & 131072 \\
\hline
\end{tabular}

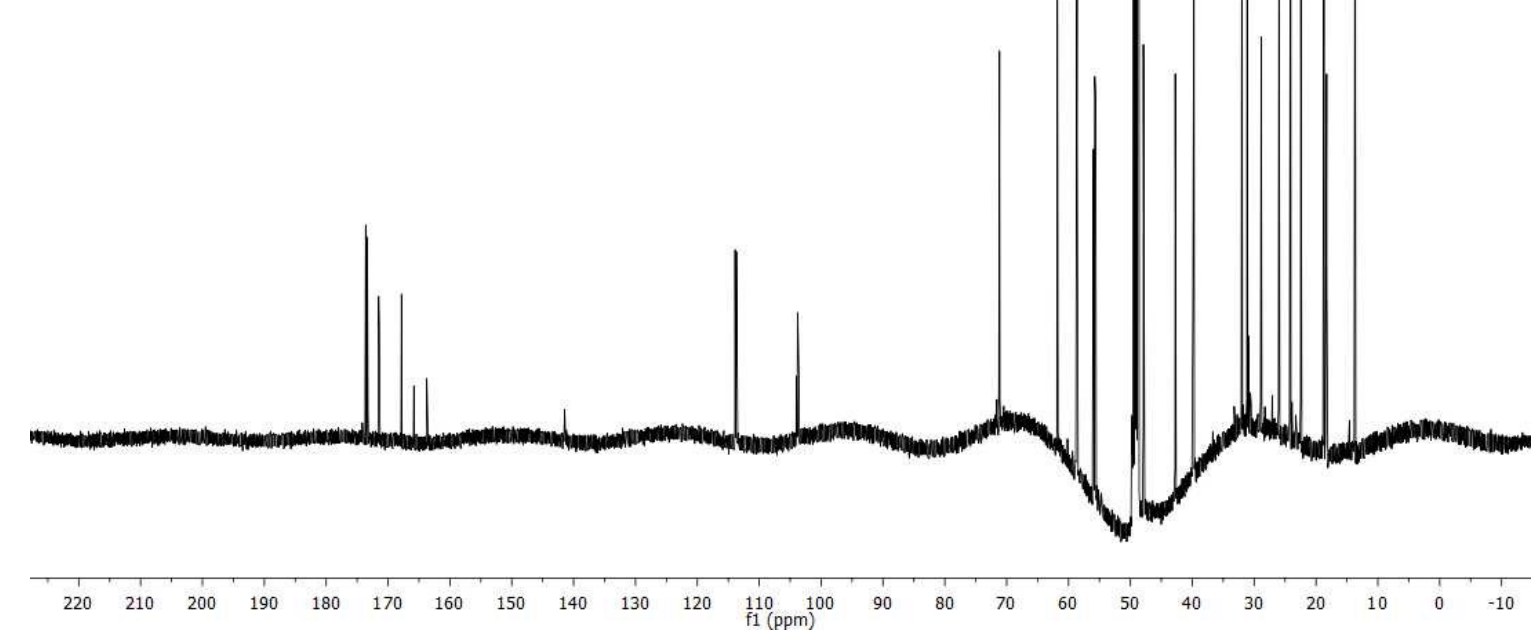




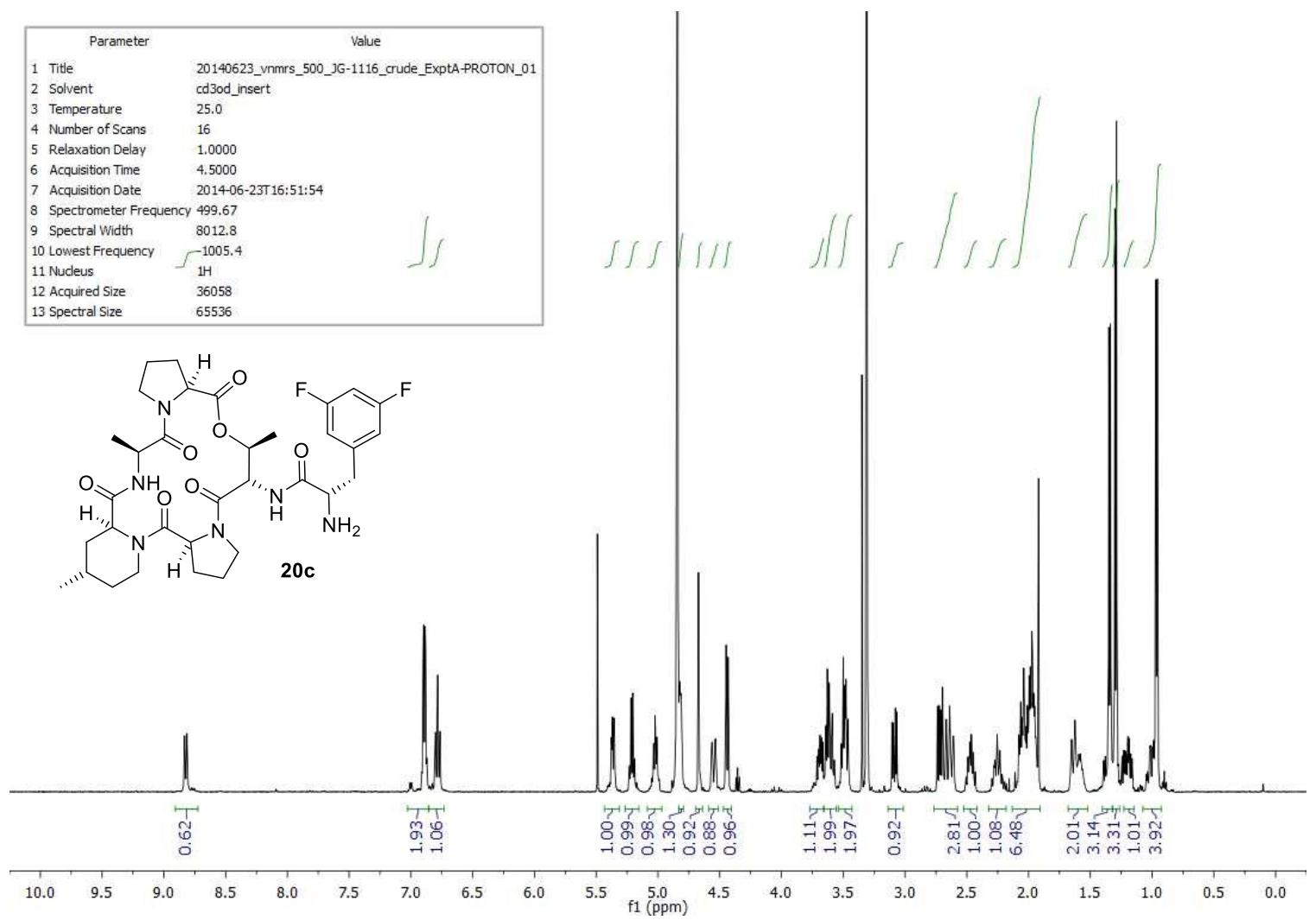

\begin{tabular}{|lll|}
\hline \multicolumn{1}{|c|}{ Parameter } & \multicolumn{1}{c|}{ Value } \\
1 & Title & 20140625 _ynmrs_500_JG-1116_crude_ExptA_2-CARBON_01 \\
2 & Solvent & cd3od_insert \\
3 & Temperature & 25.0 \\
4 & Number of Scans & 2000 \\
5 & Relaxation Delay & 0.2000 \\
6 & Acquisition Time & 2,0000 \\
7 & Acquisition Date & $2014.06-25 T 02: 24: 12$ \\
8 & Spectrometer Frequency & 125.65 \\
9 & Spectral Width & 30487.8 \\
10 & Lowest Frequency & -1854.0 \\
11 & Nucleus & $13 C$ \\
12 & Acquired Size & 60976 \\
13 & Spectral Size & 131072 \\
\hline
\end{tabular}

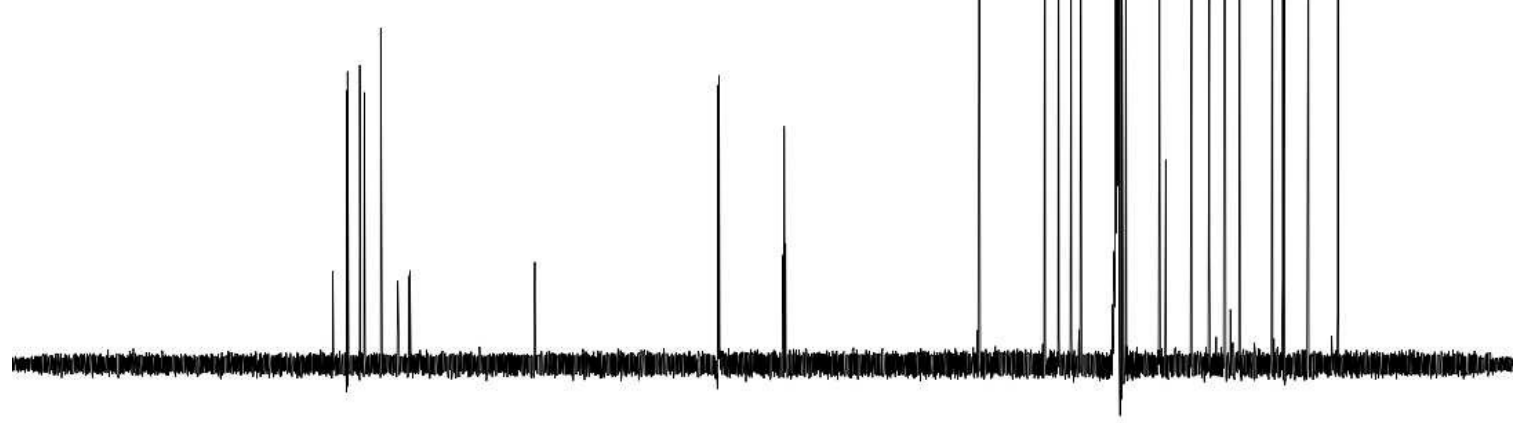

$\begin{array}{llllllllllllllllllllllllll}220 & 210 & 200 & 190 & 180 & 170 & 160 & 150 & 140 & 130 & 120 & \underset{\mathrm{f} 1}{110}(\mathrm{ppm}) & 100 & 90 & 80 & 70 & 60 & 50 & 40 & 30 & 20 & 10 & 0 & -10\end{array}$ 


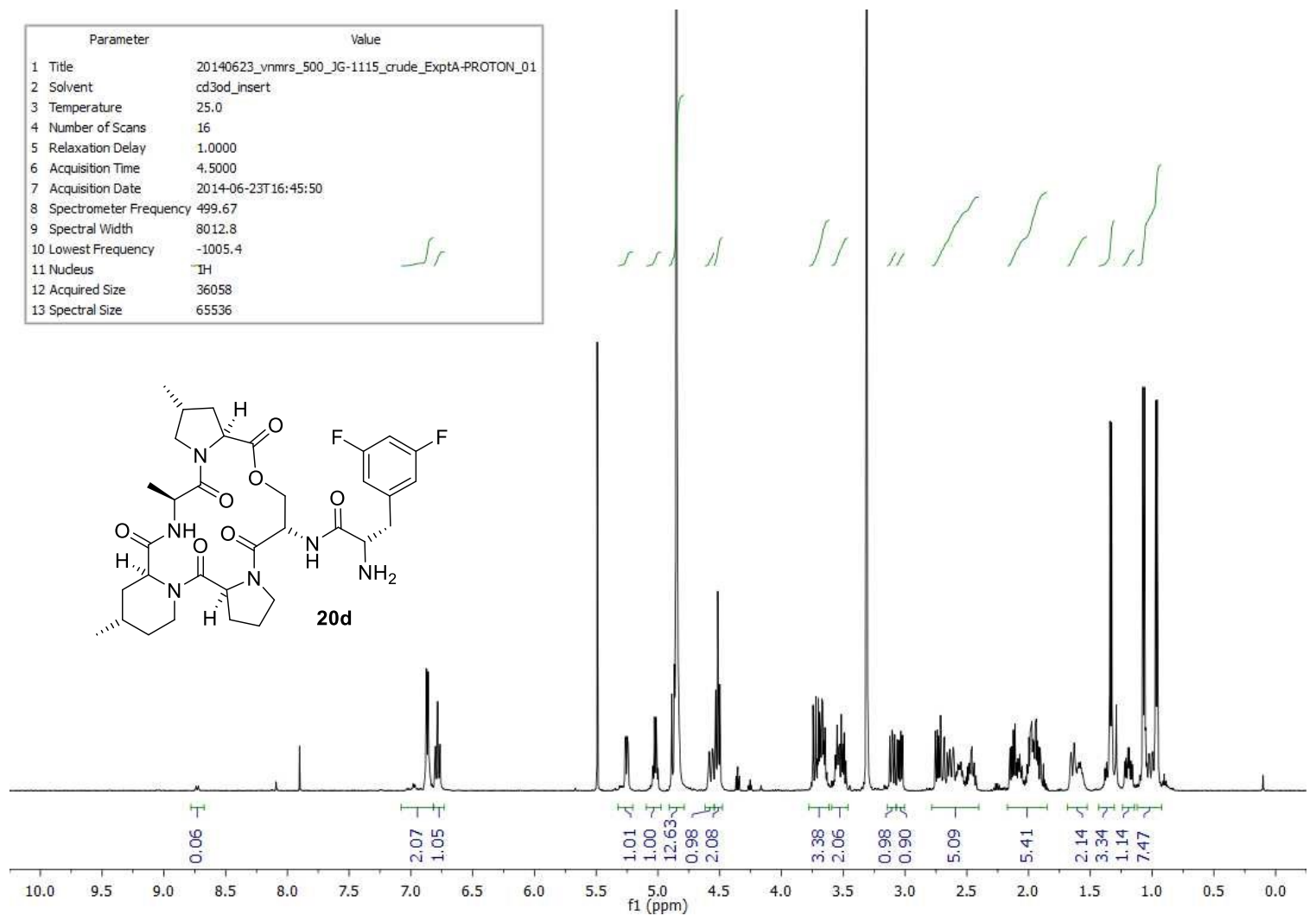

\begin{tabular}{|lll|}
\hline \multicolumn{1}{|c|}{ Parameter } & \multicolumn{1}{c|}{ Value } \\
1 & Title & 20140625 _ynmrs_500_JG-1115_crude_ExptA_2-CARBON_01 \\
2 & Solvent & cd3od_insert \\
3 & Temperature & 25.0 \\
4 & Number of Scans & 2000 \\
5 & Relaxation Delay & 0.2000 \\
6 & Acquisition Time & 2,0000 \\
7 & Acquisition Date & $201406-25 T 01: 06: 40$ \\
8 & Spectrometer Frequency & 125.65 \\
9 & Spectral Width & 30487.8 \\
10 & Lowest Frequency & -1853.3 \\
11 & Nucleus & $13 C$ \\
12 & Acquired Size & 60976 \\
13 & Spectral Size & 131072 \\
\hline
\end{tabular}

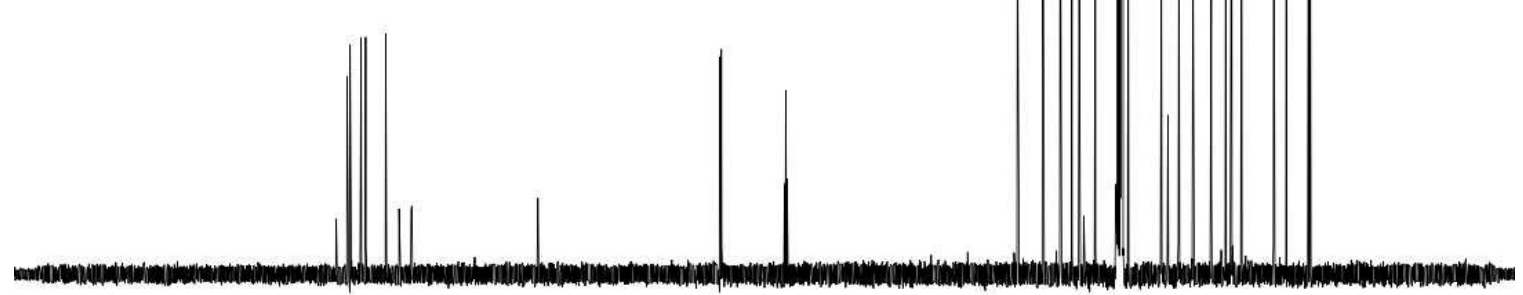

$\begin{array}{lllllllllllllllllllllllllll}220 & 210 & 200 & 190 & 180 & 170 & 160 & 150 & 140 & 130 & 120 & \underset{\mathrm{f} 1}{11}(\mathrm{ppm}) & 100 & 90 & 80 & 70 & 60 & 50 & 40 & 30 & 20 & 10 & 0 & -10\end{array}$ 


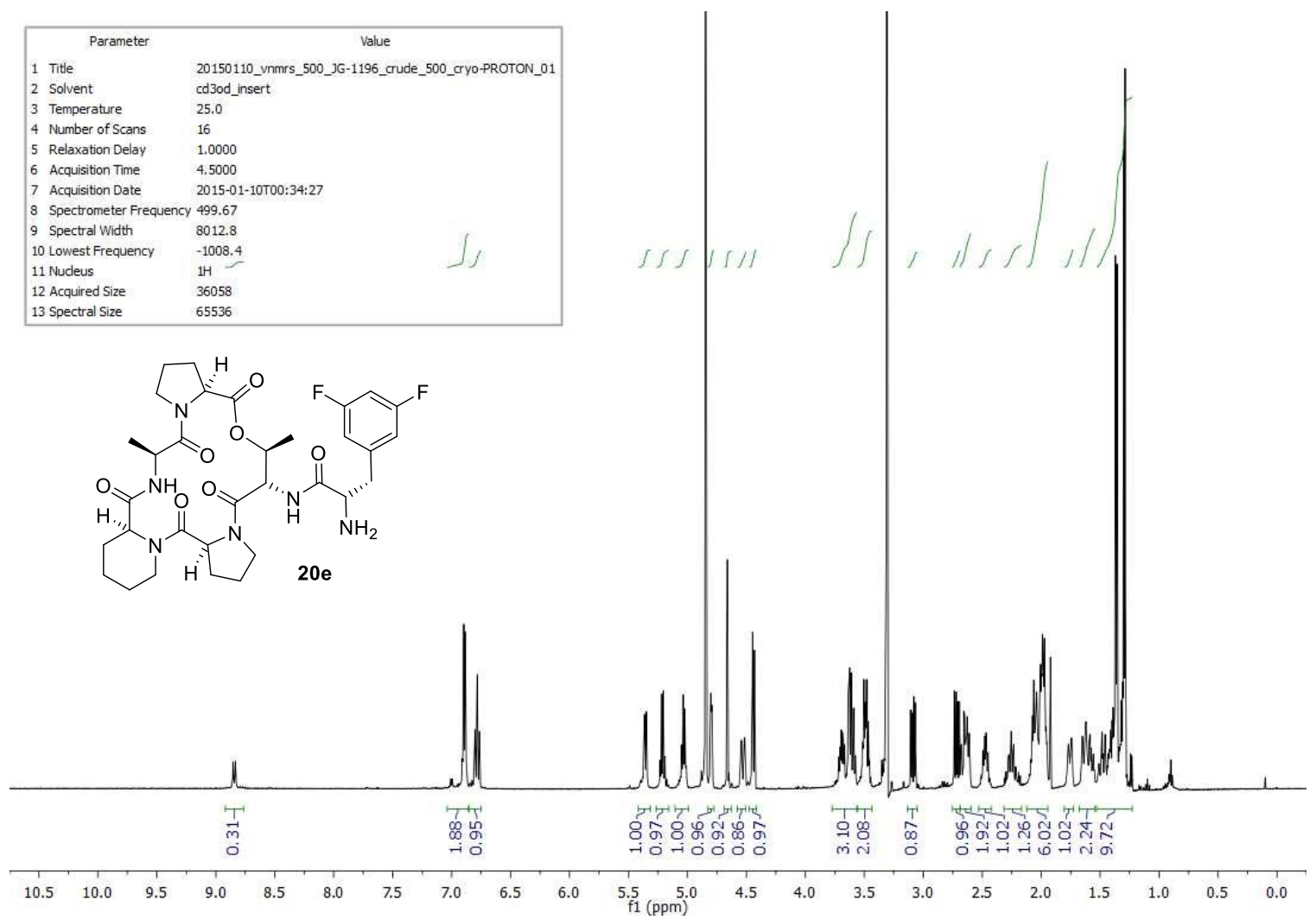

\begin{tabular}{|lll|}
\hline \multicolumn{1}{|c|}{ Parameter } & \multicolumn{1}{c|}{ Value } \\
1 & Title & $20150110 \_$ynmrs_500_JG-1196_crude_500_Cryo-CARBON_01 \\
2 & Solvent & cd3od_insert \\
3 & Temperature & 25.0 \\
4 & Number of Scans & 3000 \\
5 & Relaxation Delay & 1.0000 \\
6 & Acquisition Time & 2.0000 \\
7 & Acquisition Date & $2015-01-10 T 00: 36: 22$ \\
8 & Spectrometer Frequency 125.65 \\
9 & Spectral Width & 30487.8 \\
10 & Lowest Frequency & -1853.3 \\
11 Nucleus & $13 C$ \\
12 Acquired Size & 60976 \\
13 & Spectral Size & 131072 \\
\hline
\end{tabular}

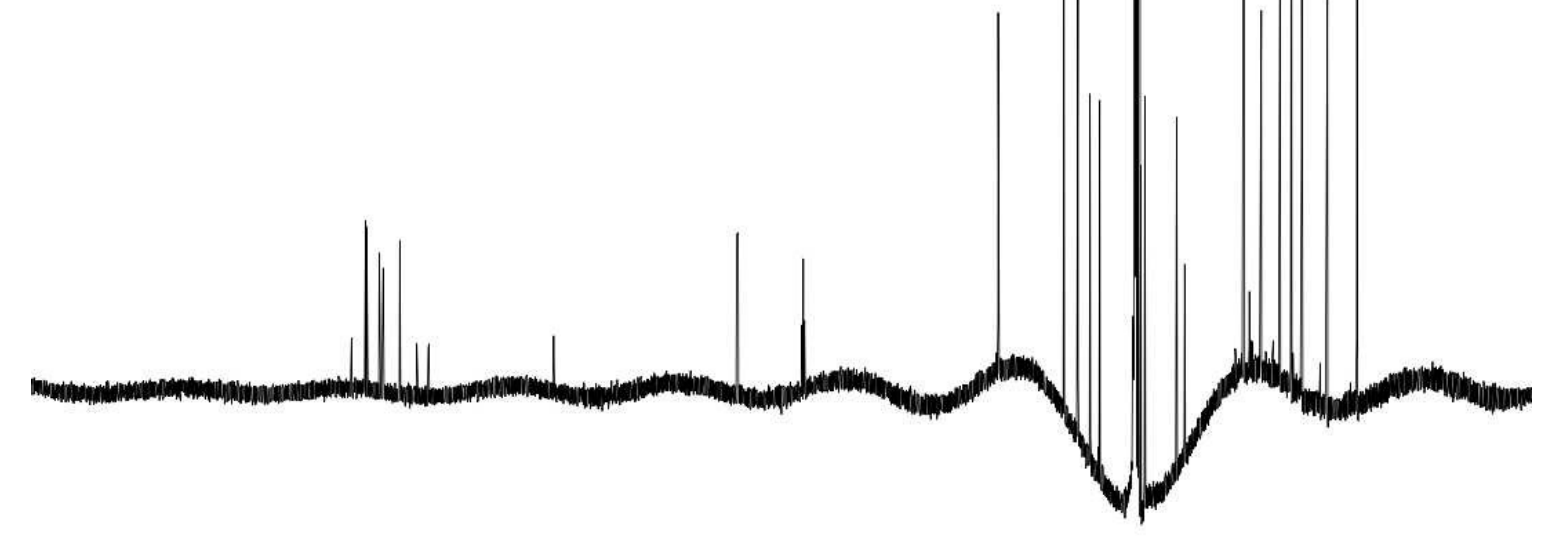

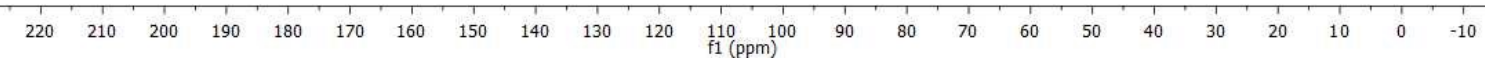




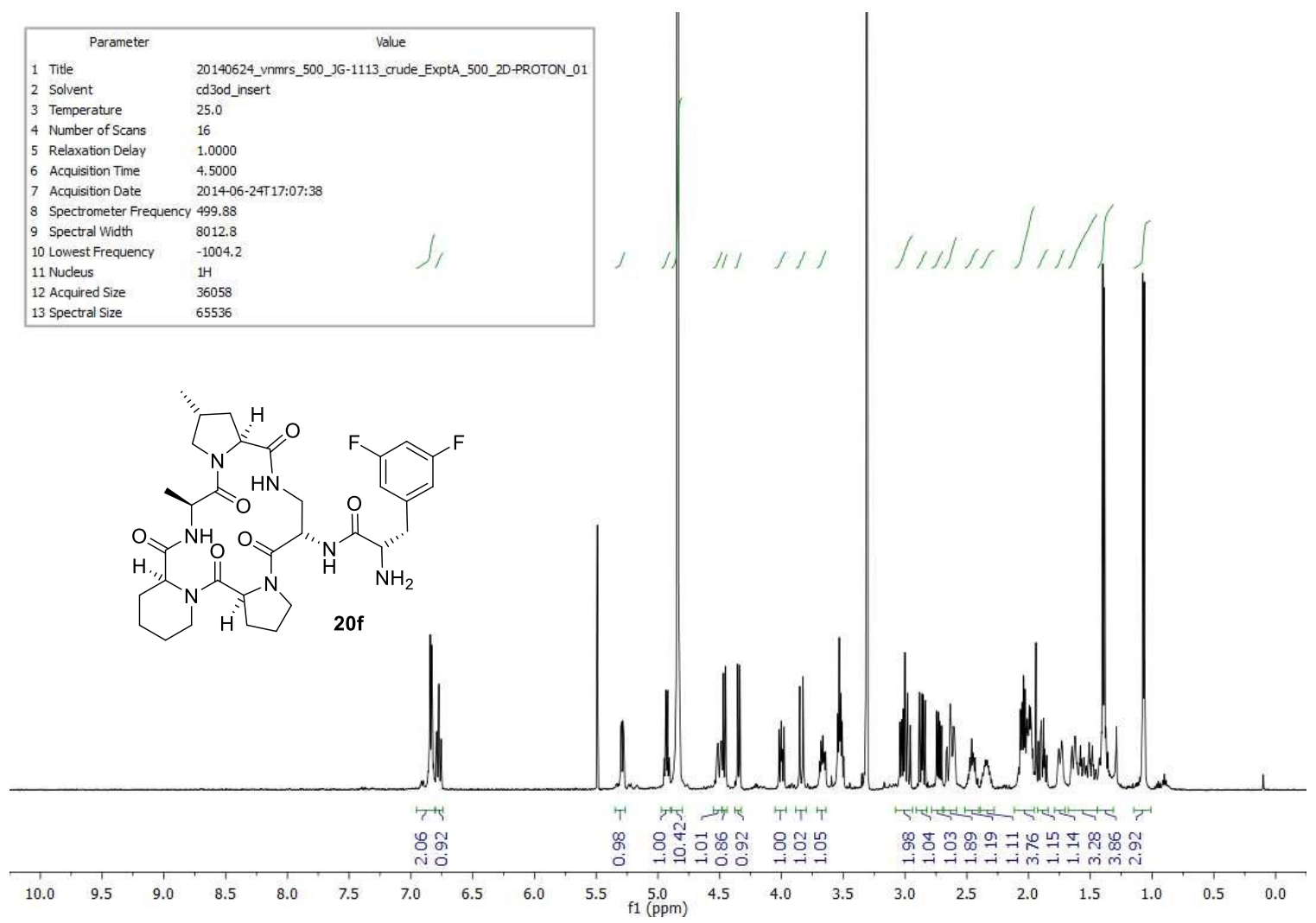

\begin{tabular}{|c|c|}
\hline Parameter & Value \\
\hline 1 Title & 20140624_ynmrs_500_JG-1113_crude_ExptA_2-CARBON_01 \\
\hline 2 Solvent & cd3od_insert \\
\hline 3 Temperature & 25.0 \\
\hline 4 Number of Scans & 2000 \\
\hline 5 Relaxation Delay & 0.2000 \\
\hline 6 Acquisition Time & 2,0000 \\
\hline 7 Acquisition Date & $2014-06-24 T 22: 30: 40$ \\
\hline 8 Spectrometer Frequency & 125.65 \\
\hline 9 Spectral Width & 30487.8 \\
\hline 10 Lowest Frequency & -2051.7 \\
\hline 11 Nucleus & $13 \mathrm{C}$ \\
\hline 12 Acquired Size & 60976 \\
\hline 13 Spectral Size & 131072 \\
\hline
\end{tabular}

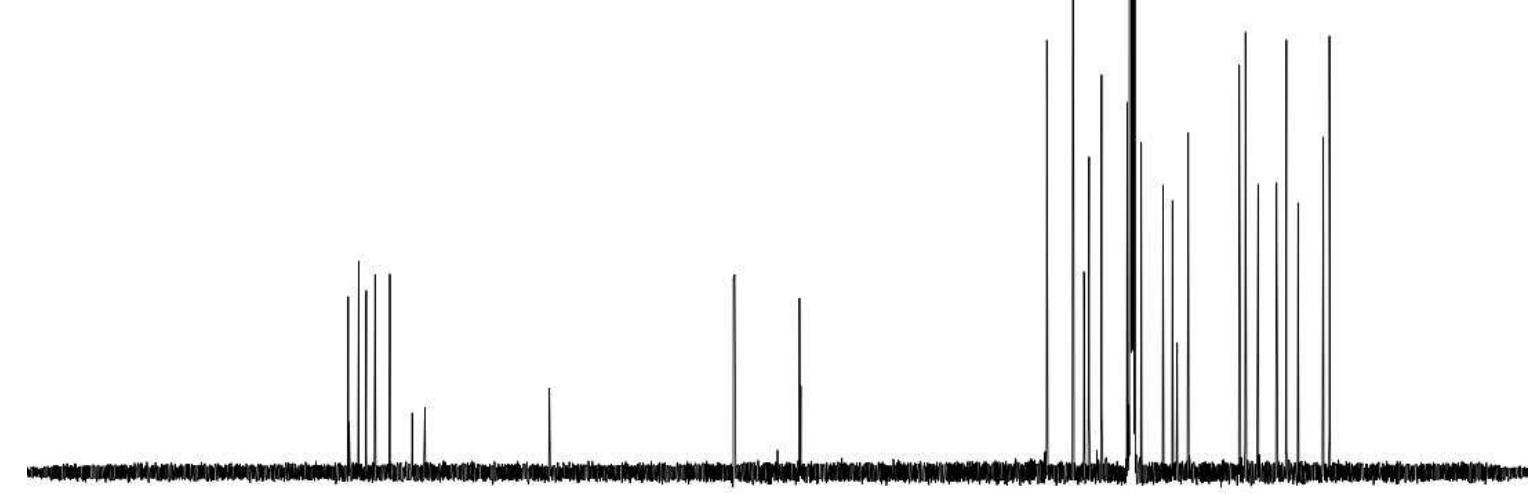

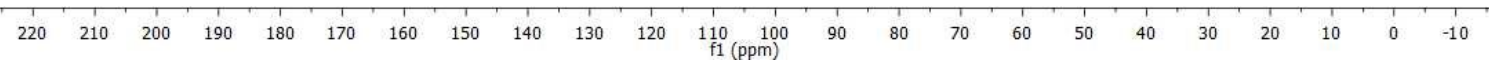




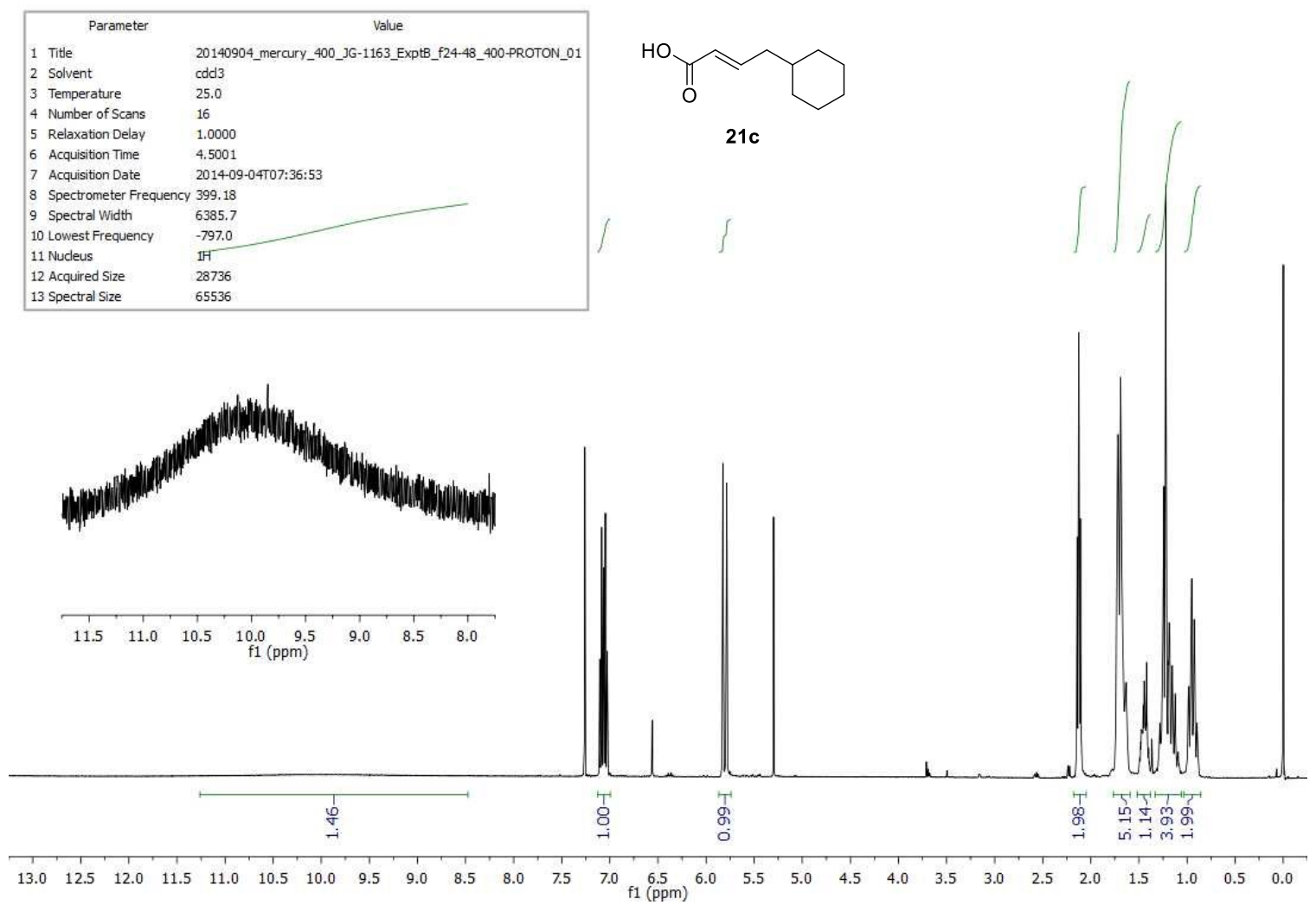

\begin{tabular}{|c|c|}
\hline Parameter & Value \\
\hline 1 Title & 20140904 mercury_400_JG-1163_ExptB_f24-48_400-CARBON_01 \\
\hline 2 Solvent & $\operatorname{cdd} 3$ \\
\hline 3 Temperature & 25.0 \\
\hline 4 Number of Scans & 1000 \\
\hline 5 Relaxation Delay & 1.0000 \\
\hline 6 Acquisition Time & 2.0000 \\
\hline 7 Acquisition Date & 2014-09-04T07:39:14 \\
\hline 8 Spectrometer Frequency & 100.38 \\
\hline 9 Spectral Width & 25062.7 \\
\hline 10 Lowest Frequency & -1473.0 \\
\hline 11 Nucleus & $13 \mathrm{C}$ \\
\hline 12 Acquired Size & 50125 \\
\hline 13 Spectral Size & 131072 \\
\hline
\end{tabular}

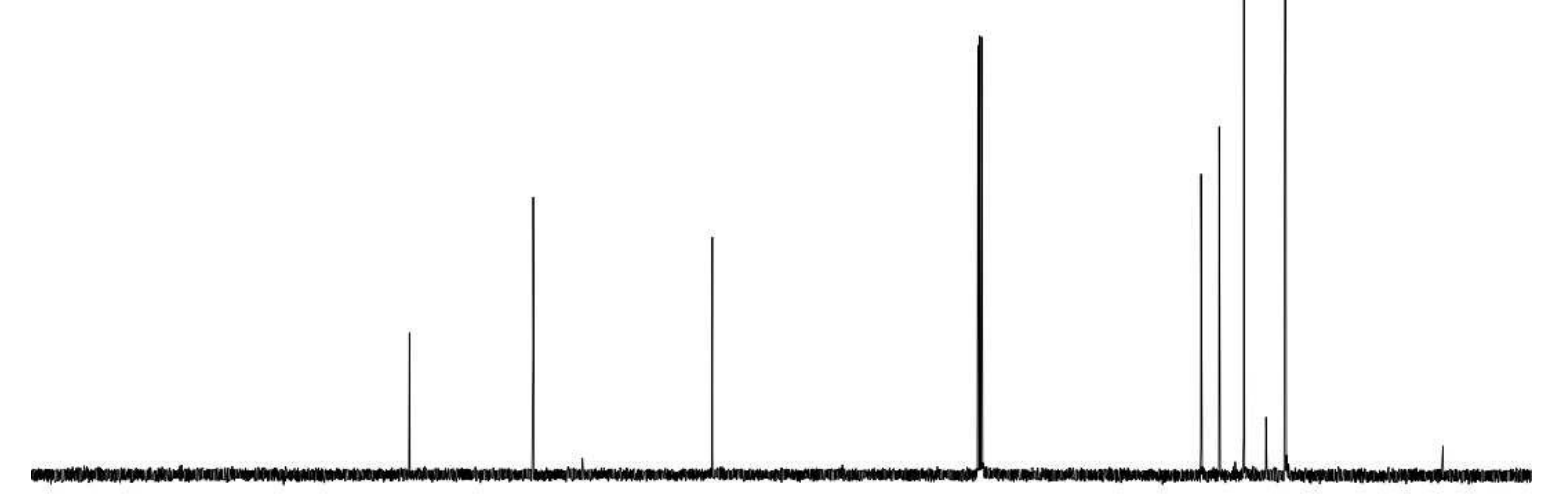

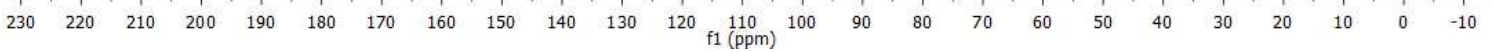




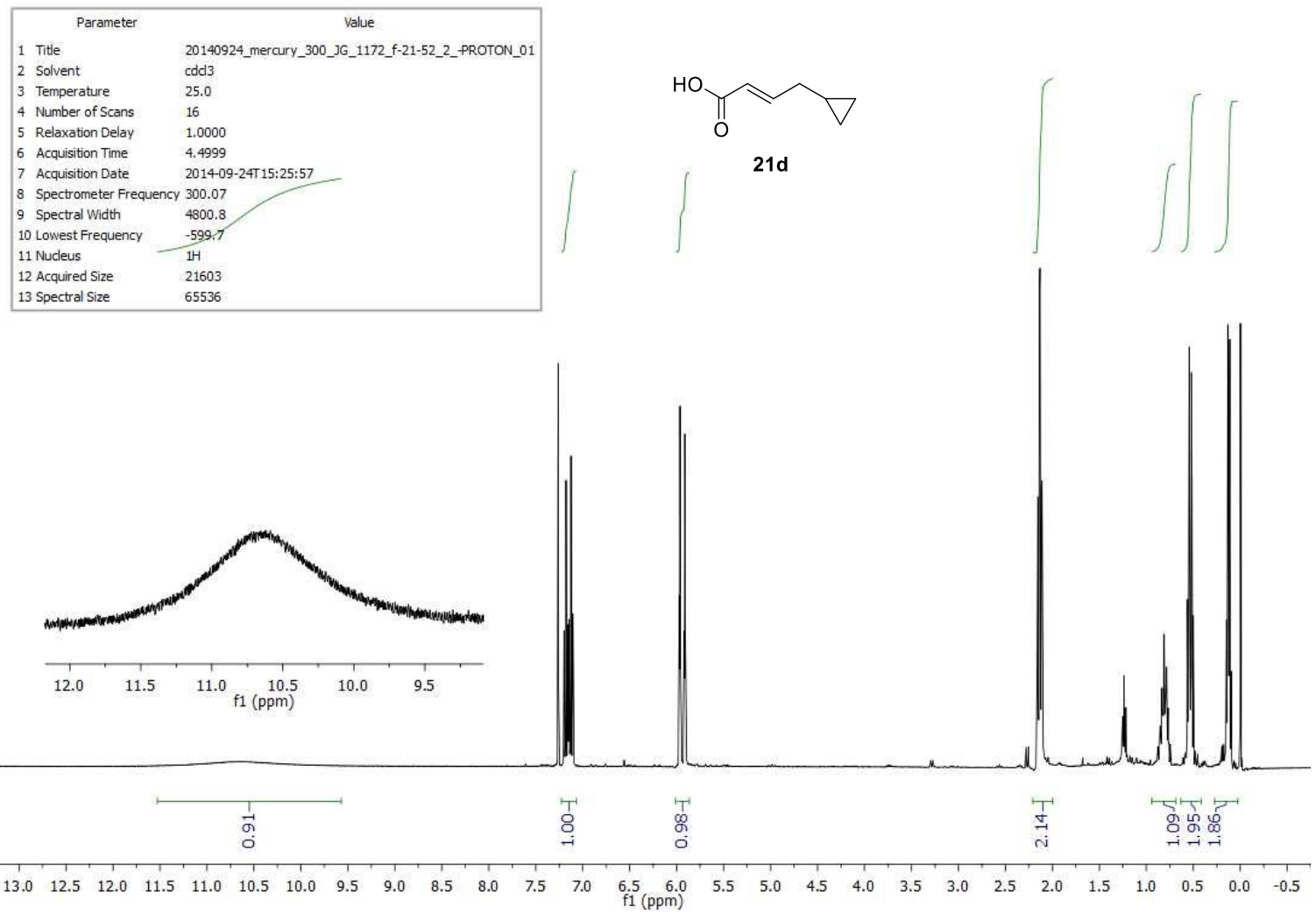

\begin{tabular}{|lll|}
\hline \multicolumn{1}{|c|}{ Parameter } \\
1 Title & 20141003 mercury_400_JG1172_2_f_21-52-CARBON_01 \\
2 & Solvent & Cdd3 \\
3 & Temperature & 25.0 \\
4 & Number of Scans & 2000 \\
5 & Relaxation Delay & 0.2000 \\
6 & Acquisition Time & 2.0000 \\
7 & Acquisition Date & $2014-10-03 T 01: 45: 55$ \\
8 & Spectrometer Frequency 100.38 \\
9 & Spectral Width & 25062.7 \\
10 & Lowest Frequency & -1473.4 \\
11 & Nudeus & $13 C$ \\
12 Acquired Size & 50125 \\
13 Spectral Size & 131072 \\
\hline
\end{tabular}

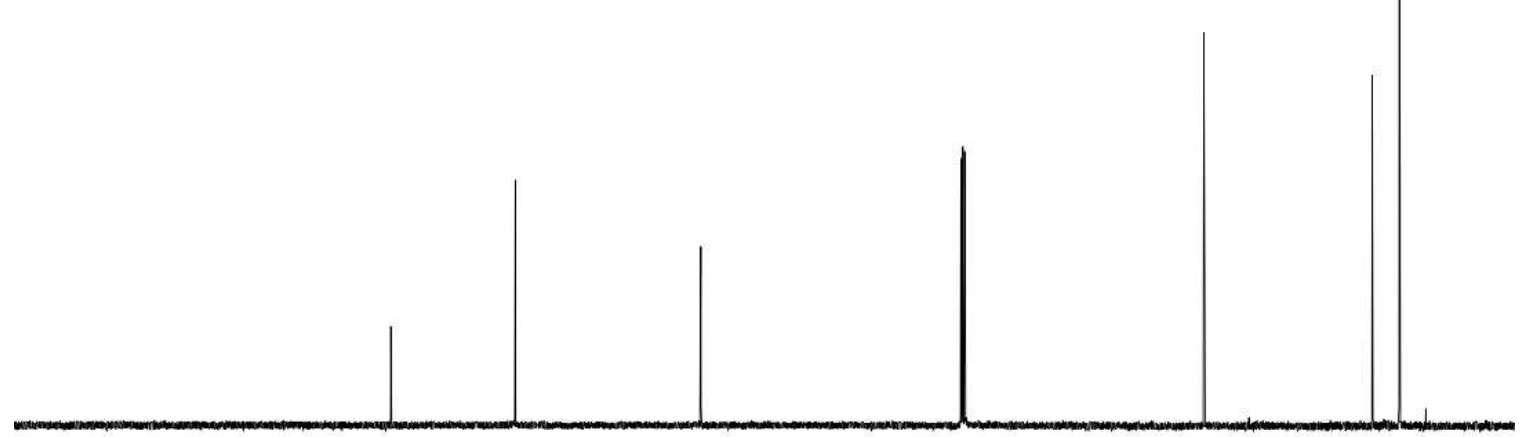

$\begin{array}{lllllllllllllllllllllllllllllllllll}230 & 220 & 210 & 200 & 190 & 180 & 170 & 160 & 150 & 140 & 130 & 120 & 110 & 100 & 90 & 80 & 70 & 60 & 50 & 40 & 30 & 20 & 10 & 0 & -10\end{array}$ 


\begin{tabular}{|lll|}
\hline \multicolumn{1}{|c|}{ Parameter } & \multicolumn{1}{c|}{ Value } \\
1 & Title & 20140918 ynmrs_400_JG-1171_f37-68_rerun3_400-PROTON_01 \\
2 & Solvent & cdd3 \\
3 & Temperature & 25.3 \\
4 & Number of Scans & 16 \\
5 & Relaxation Delay & 1.0000 \\
6 & Acquisition Time & 4.5000 \\
7 & Acquisition Date & $2014-09-18 T 01: 01: 46$ \\
8 & Spectrometer Frequency & 399.74 \\
9 & Spectral Width & 6410.3 \\
10 & Lowest Frequency & -806.7 \\
11 & Nucleus & $1 H$ \\
12 & Acquired Size & 28846 \\
13 & Spectral Size & 65536 \\
\hline
\end{tabular}
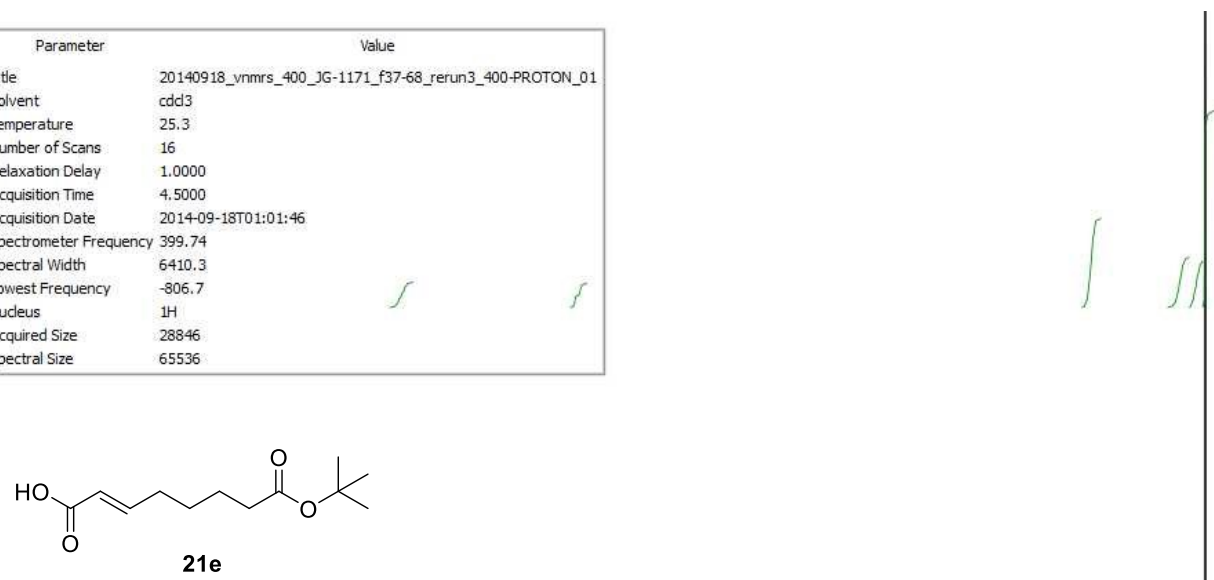

21e

\begin{tabular}{|lll|}
\hline \multicolumn{1}{|c|}{ Parameter } & \multicolumn{1}{c|}{ Value } \\
1 & Title & 20140919_ynmrs_400_JG-1171_f37-68_13C-CARBON_01 \\
2 & Solvent & cd3od_insert \\
3 & Temperature & 25.3 \\
4 & Number of Scans & 1000 \\
5 & Relaxation Delay & 0.2000 \\
6 & Acquisition Time & 1.3631 \\
7 & Acquisition Date & $201409-19701: 56: 52$ \\
8 & Spectrometer Frequency 100.53 \\
9 & Spectral Width & 24038.5 \\
10 & Lowest Frequency & -1050.3 \\
11 Nucleus & $13 C$ \\
12 Acquired Size & 32768 \\
13 Spectral Size & 65536 \\
\hline
\end{tabular}

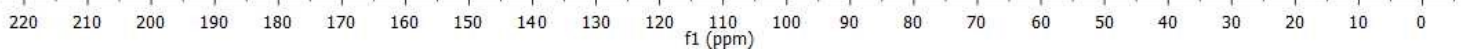



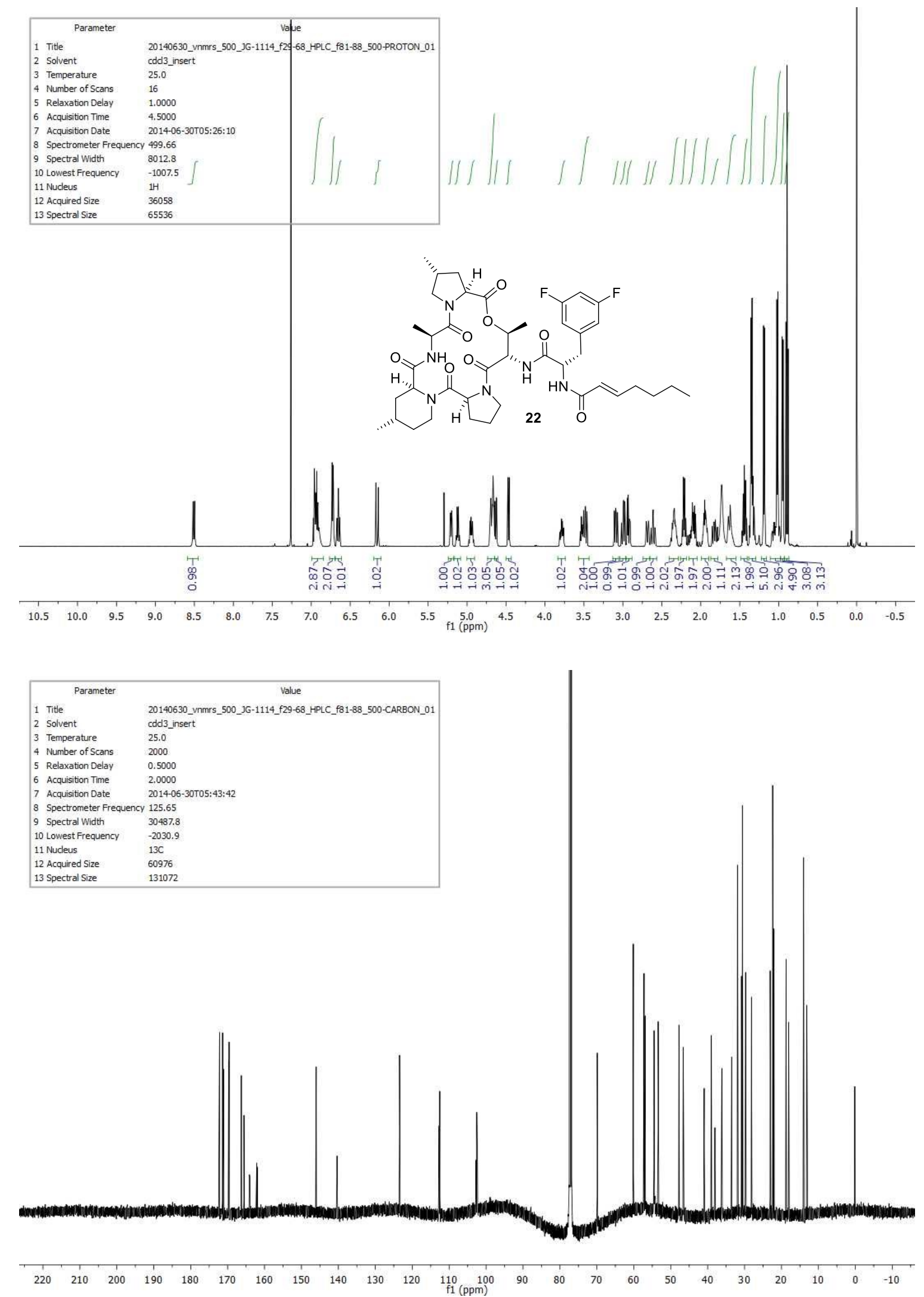


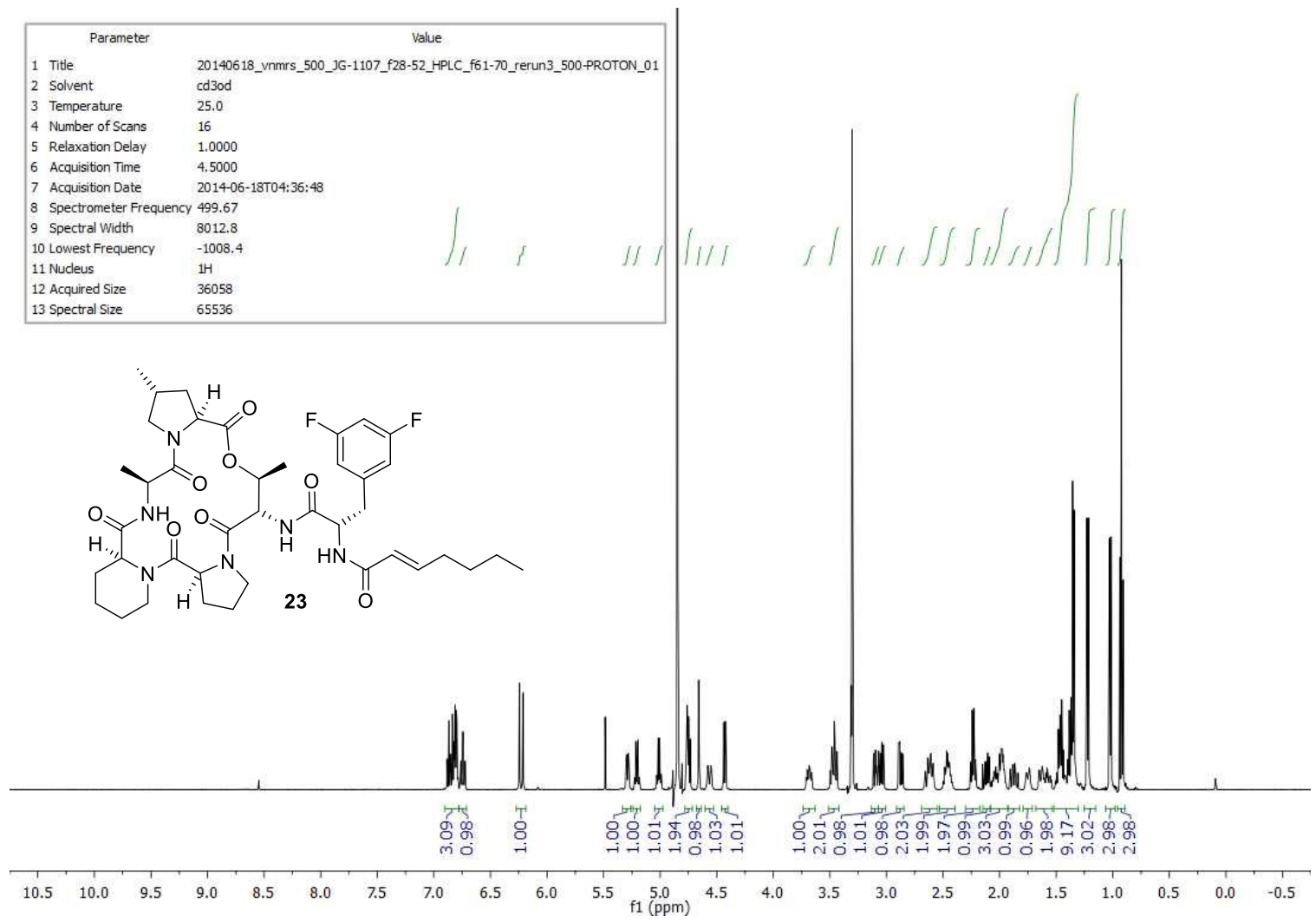

\begin{tabular}{|lll|}
\hline \multicolumn{1}{|c|}{ Parameter } & \multicolumn{1}{c|}{ Value } \\
1 & Tite & $20140618 \_$ynmrs_500_JG-1107_f28-52_HPLC_f61-70_rerun3_500-CARBON_01 \\
2 & Solvent & cd30d \\
3 & Temperature & 25.0 \\
4 & Number of Scans & 2000 \\
5 & Relaxation Delay & 1.0000 \\
6 & Acquisition Time & 2.0000 \\
7 & Acquisition Date & $2014-06-18 T 04: 38: 42$ \\
8 & Spectrometer Frequency & 125.65 \\
9 & Spectral Width & 30487.8 \\
10 & Lowest Frequency & -1853.5 \\
11 & Nucleus & $13 \mathrm{C}$ \\
12 & Acquired Size & 60976 \\
13 & Spectral Size & 131072 \\
\hline
\end{tabular}

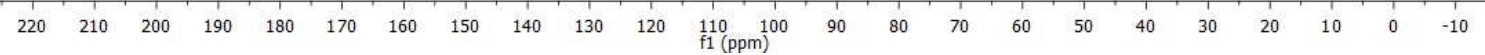



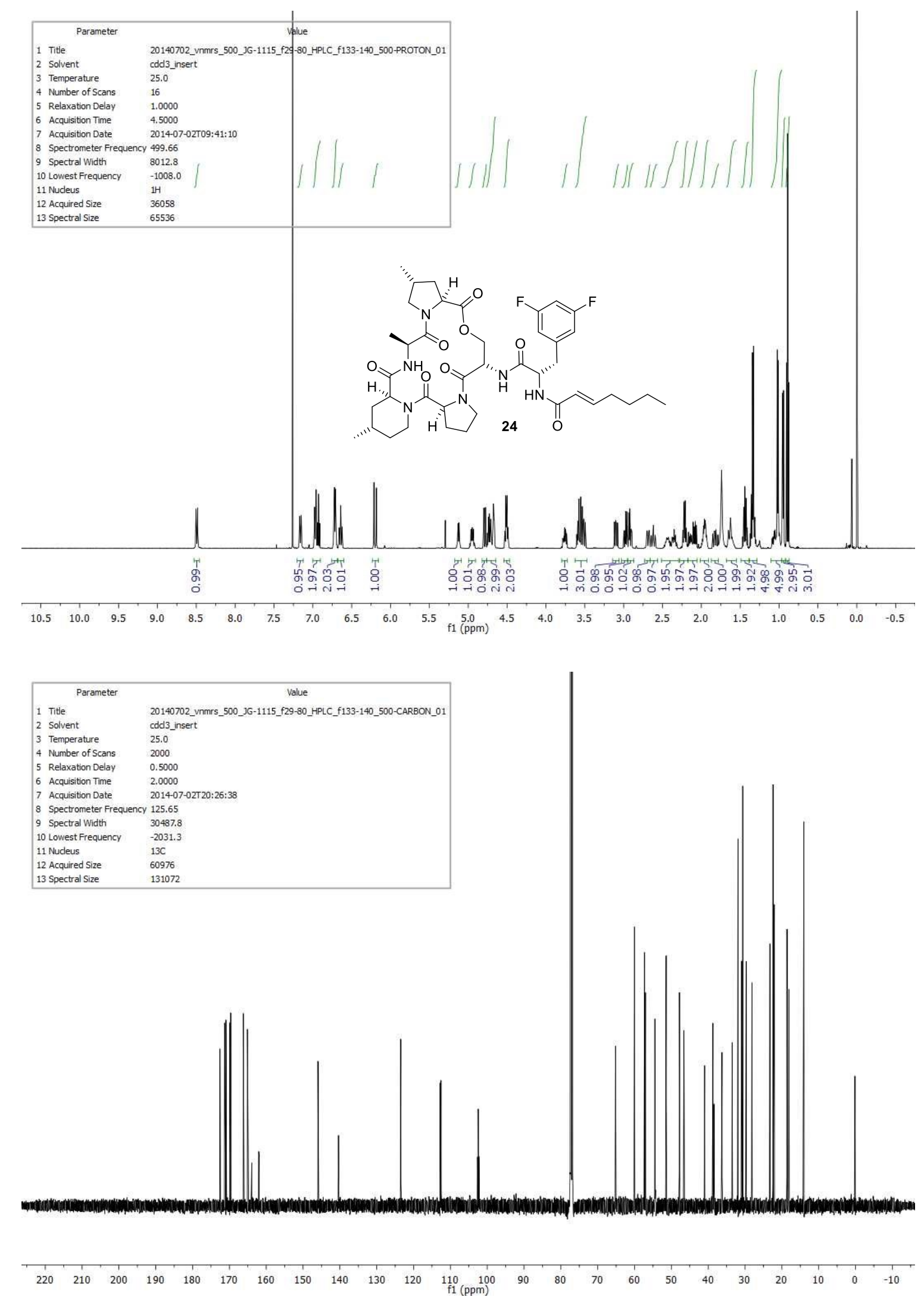


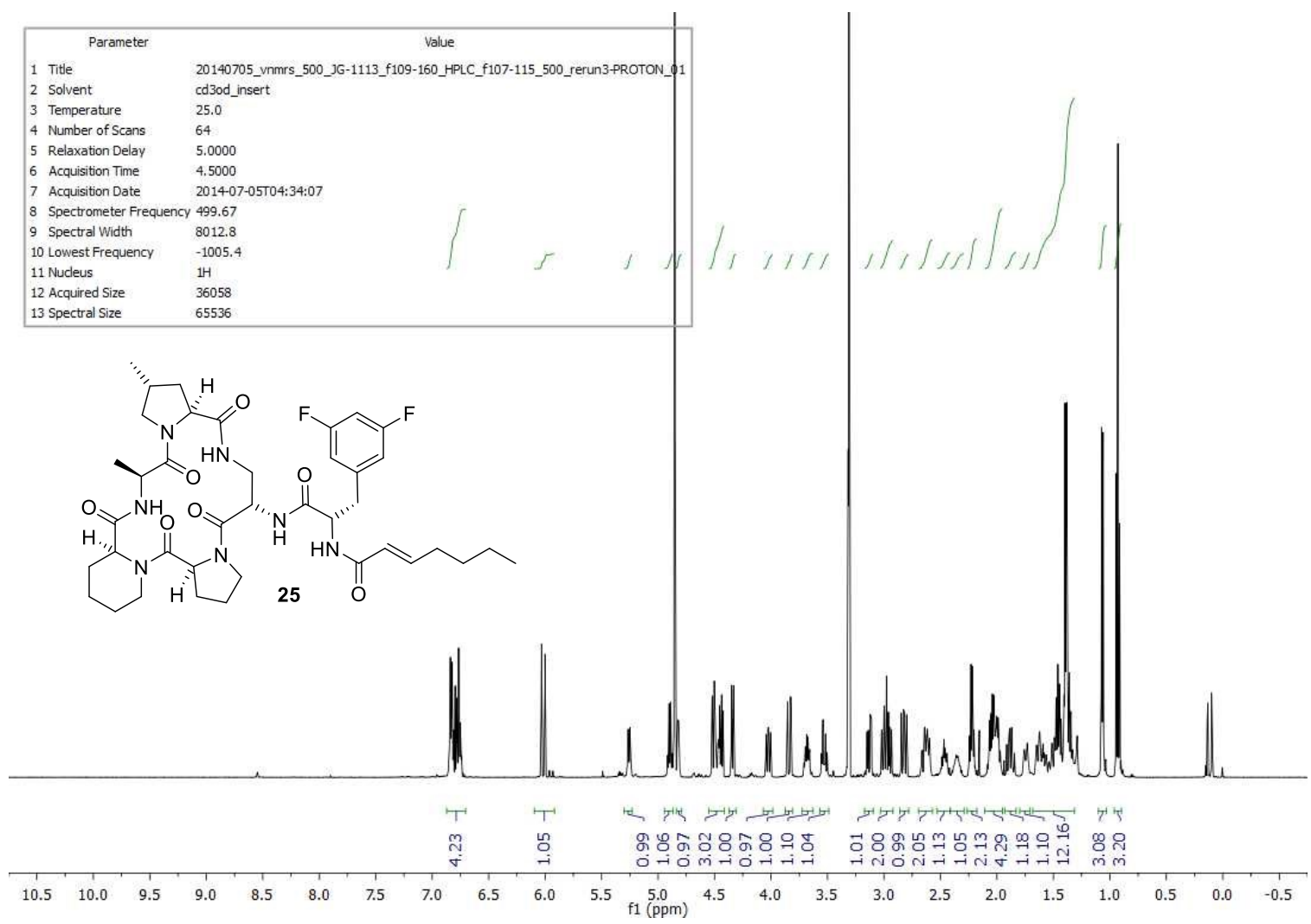

\begin{tabular}{|c|c|}
\hline Parameter & Value \\
\hline 1 Title & 20140705_vnmrs_500_JG-1113_f109-160_HPLC_f107-115_500_rerun3-CARBON_01 \\
\hline 2 Solvent & cd3od_insert \\
\hline 3 Temperature & 25.0 \\
\hline 4 Number of Scans & 3000 \\
\hline 5 Relaxation Delay & 1.0000 \\
\hline 6 Acquisition Time & 2,0000 \\
\hline 7 Acquisition Date & 2014-07-05T04:44:50 \\
\hline 8 Spectrometer Frequency & 125.65 \\
\hline 9 Spectral Width & 30487.8 \\
\hline 10 Lowest Frequency & -1853.4 \\
\hline 11 Nucleus & $13 \mathrm{C}$ \\
\hline 12 Acquired Size & 60976 \\
\hline 13 Spectral Size & 131072 \\
\hline
\end{tabular}

$\begin{array}{llllllllllllllllllllllll}220 & 210 & 200 & 190 & 180 & 170 & 160 & 150 & 140 & 130 & 120 & \underset{\mathrm{f1}}{110}(\mathrm{ppm}) & 100 & 90 & 80 & 70 & 60 & 50 & 40 & 30 & 20 & 10 & 0 & -10\end{array}$ 


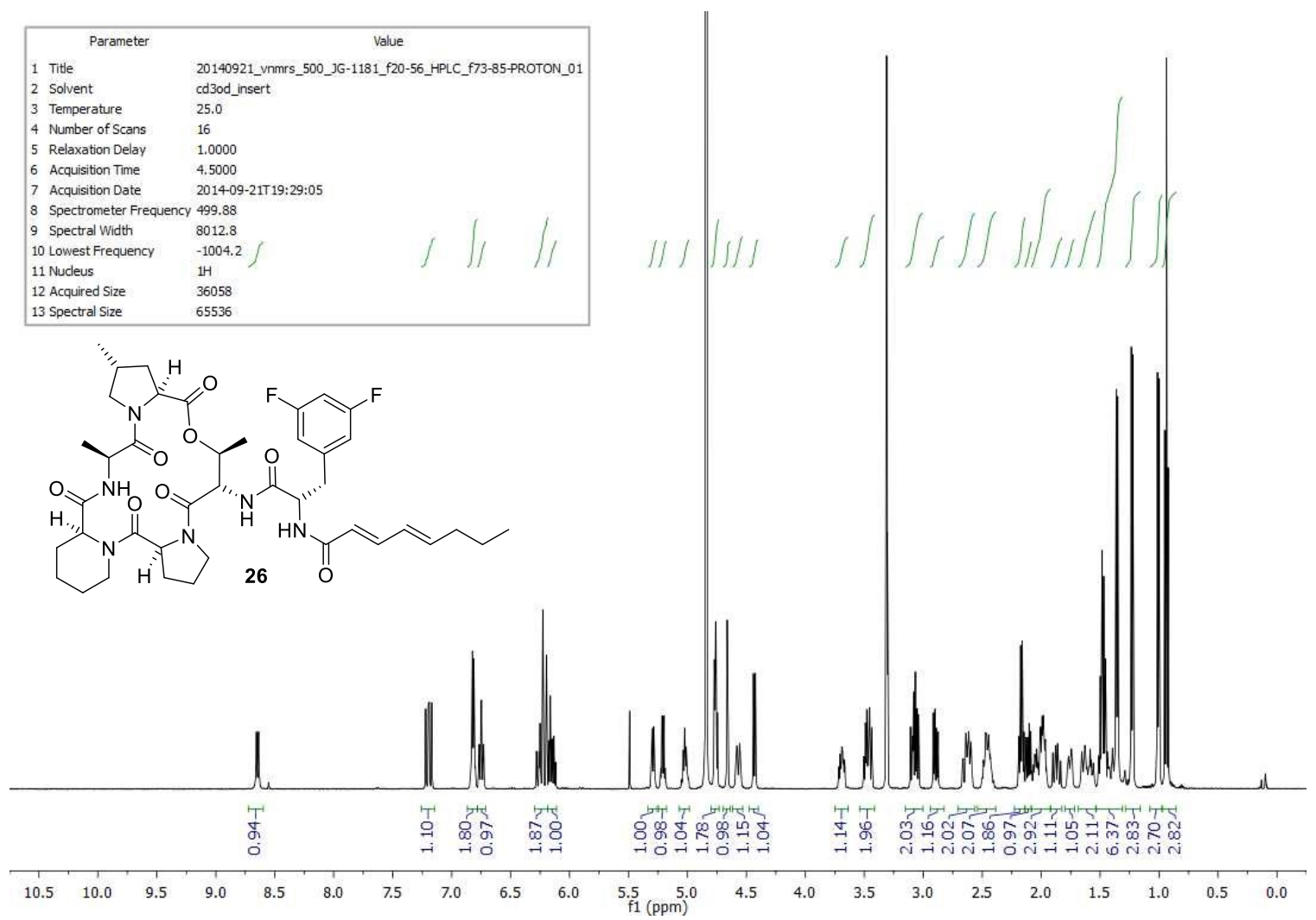

\begin{tabular}{|c|c|}
\hline Parameter & Value \\
\hline 1 Title & 20140922_ynmrs_500_JG-1181_f20-56_HPLC_f73-85-CARBON_01 \\
\hline 2 Solvent & cd3od_insert \\
\hline 3 Temperature & 25.0 \\
\hline 4 Number of Scans & 10000 \\
\hline 5 Relaxation Delay & 0.2000 \\
\hline 6 Acquisition Time & 2,0000 \\
\hline 7 Acquisition Date & 2014-09-21T20:51:35 \\
\hline 8 Spectrometer Frequency & 125.71 \\
\hline 9 Spectral Width & 29069,8 \\
\hline 10 Lowest Frequency & -1138.7 \\
\hline 11 Nucleus & $13 \mathrm{C}$ \\
\hline 12 Acquired Size & 58140 \\
\hline 13 Spectral Size & 131072 \\
\hline
\end{tabular}
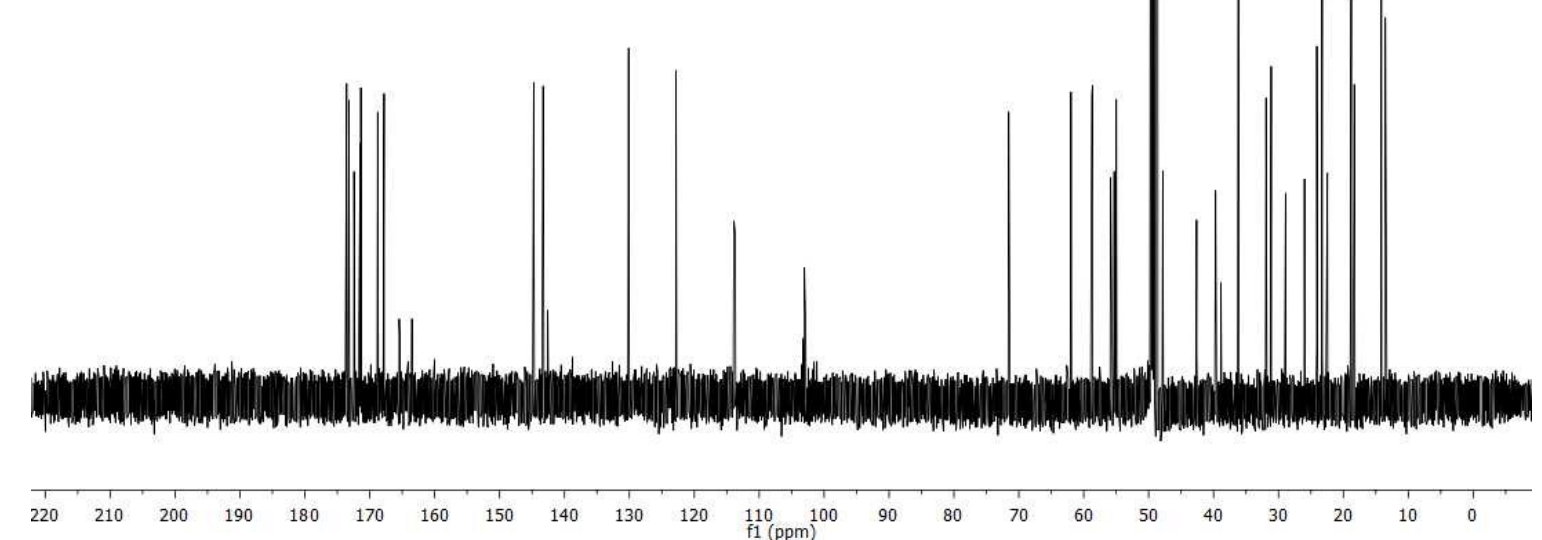

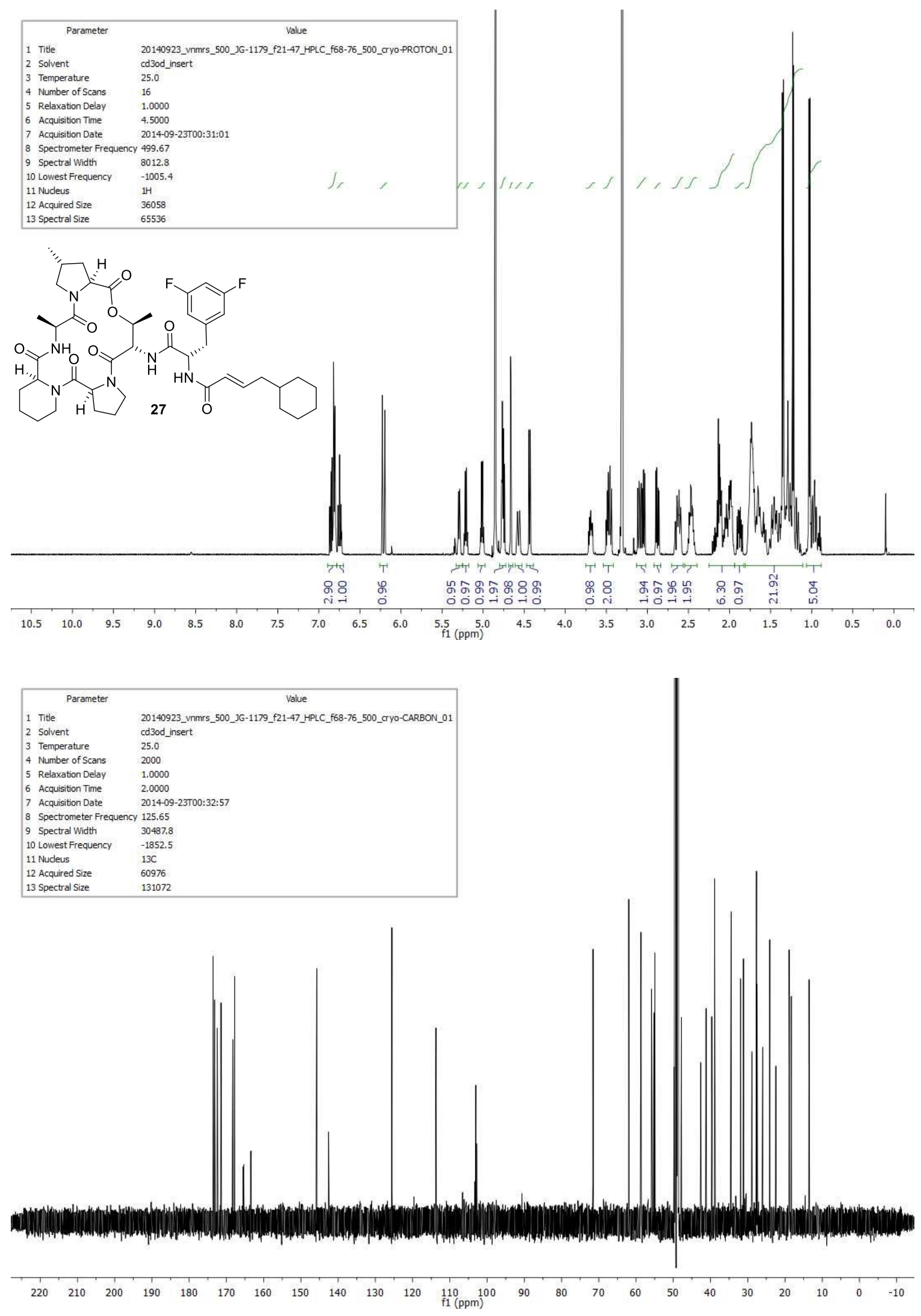

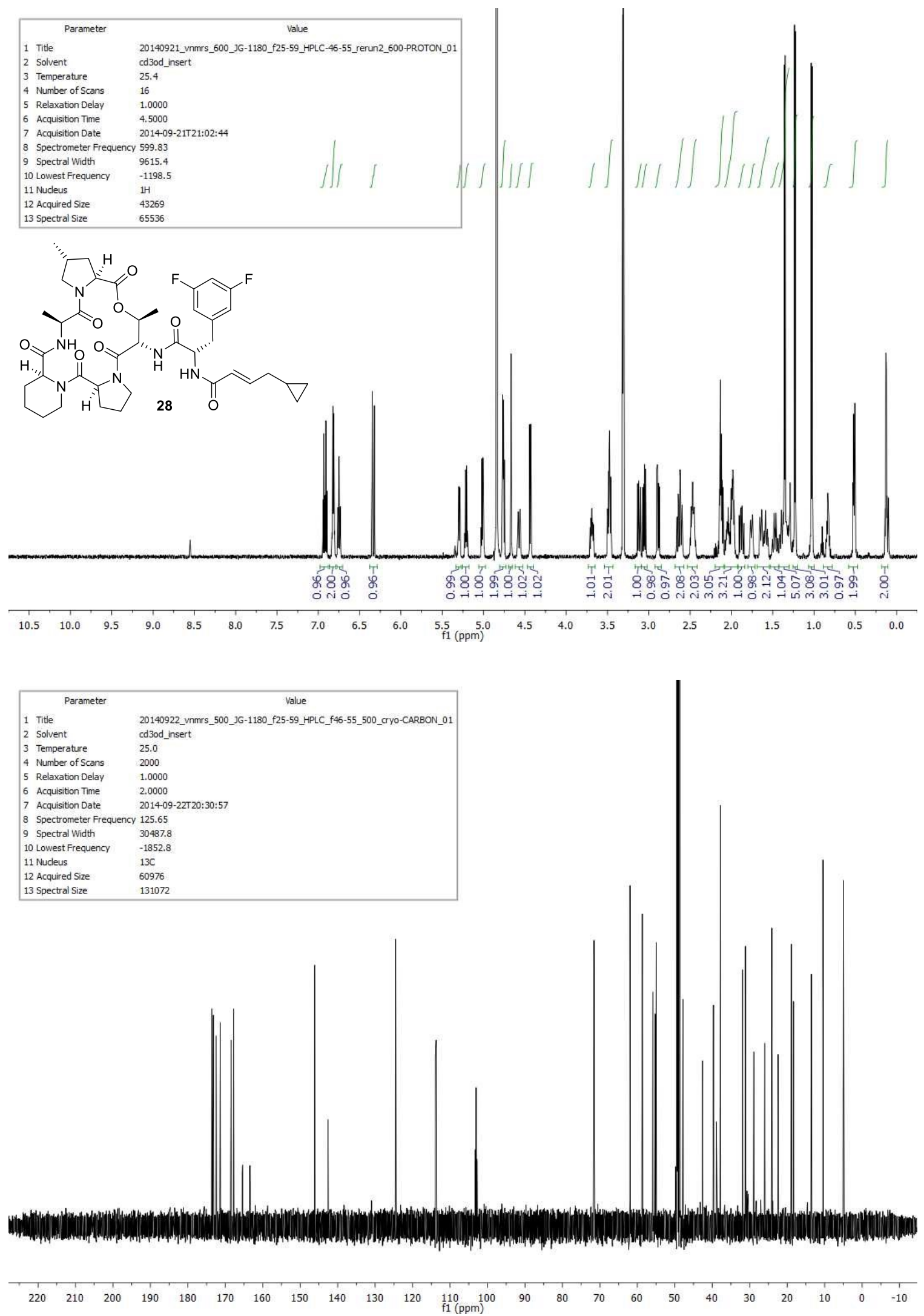


\begin{tabular}{|c|c|}
\hline Parameter & Value \\
\hline 1 Title & 20140921_ynmrs_400_JG-1183_f16-43_rerun_400-PROTON_01 \\
\hline 2 Solvent & $\operatorname{cd} 3 \circ d$ \\
\hline 3 Temperature & 25.3 \\
\hline 4 Number of Scans & 16 \\
\hline 5 Relaxation Delay & 1.0000 \\
\hline 6 Acquisition Time & 4.5000 \\
\hline 7 Acquisition Date & 2014-09-21T03:10:10 \\
\hline 8 Spectrometer Frequency & 399.75 \\
\hline 9 Spectral Width & 6410.3 \\
\hline 10 Lowest Frequency & -797.9 \\
\hline 11 Nucleus & $1 \mathrm{H}$ \\
\hline 12 Acquired Size & 28846 \\
\hline 13 Spectral Size & 65536 \\
\hline
\end{tabular}<smiles>CC1CCC(C(=O)OC(C)C(NC(=O)C(C)OC(=O)CN2CCCC2C(=O)N2CCCCC2C(=O)O)C(=O)NC(C)Cc2cc(F)cc(F)c2)C1</smiles>

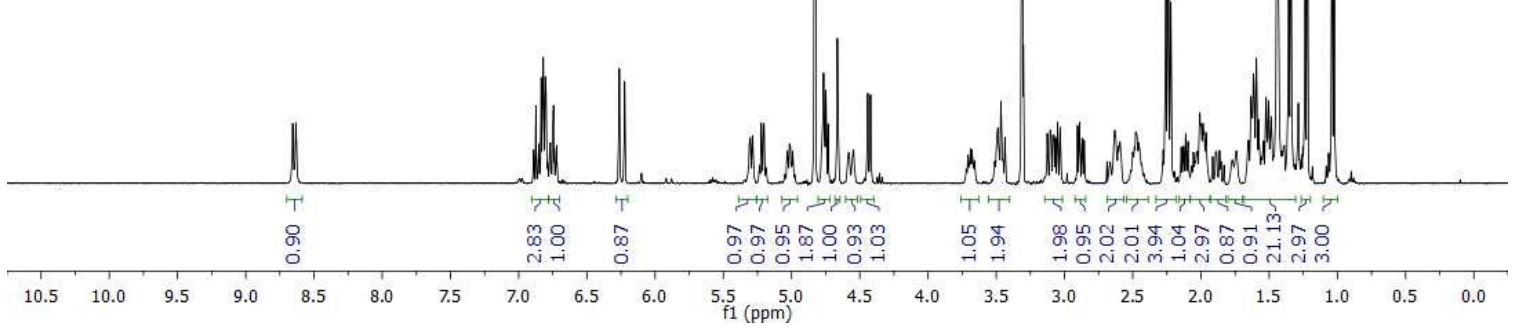

\begin{tabular}{|c|c|}
\hline Parameter & Value \\
\hline 1 Title & 20140921_ynmrs_400_JG-1183_f16-43_rerun_400-CARBON_01 \\
\hline 2 Solvent & cd3od \\
\hline 3 Temperature & 25.3 \\
\hline 4 Number of Scans & 11000 \\
\hline 5 Relaxation Delay & 1.0000 \\
\hline 6 Acquisition Time & 1.3631 \\
\hline 7 Acquisition Date & 2014-09-21T04:32:34 \\
\hline 8 Spectrometer Frequency & 100.53 \\
\hline 9 Spectral Width & 24038.5 \\
\hline 10 Lowest Frequency & -1306.2 \\
\hline 11 Nudeus & $13 \mathrm{C}$ \\
\hline 12 Acquired Size & 32768 \\
\hline 13 Spectral Size & 65536 \\
\hline
\end{tabular}

$\begin{array}{lllllllllllllllllllllllllllll}220 & 210 & 200 & 190 & 180 & 170 & 160 & 150 & 140 & 130 & 120 & 110 & 100 & 90 & 80 & 70 & 60 & 50 & 40 & 30 & 20 & 10 & 0 & -10\end{array}$ 

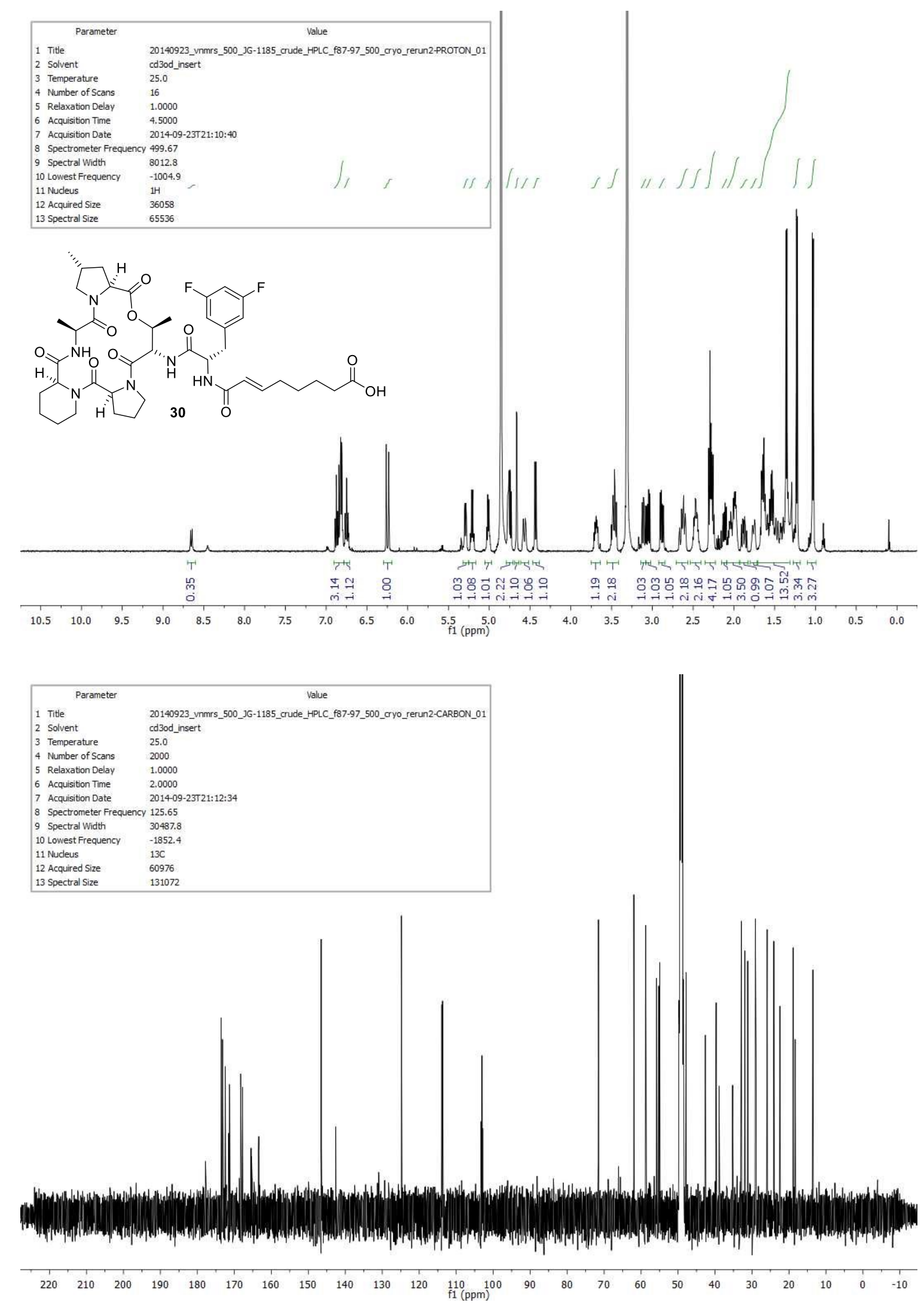


\section{HPLC Chromatograms for Numbered Compounds}

Table S1. Analytical HPLC Method.

Column:

Agilent Eclipse XDB-C18, 4.6 x 150 mm $(5 \mu \mathrm{m})$

Detector $\lambda$ :

$220 \mathrm{~nm}$

Flow rate:

$2.0 \mathrm{~mL} / \mathrm{min}$

Column temp:

$23{ }^{\circ} \mathrm{C}$

Gradient elution using solvents: $\mathrm{H}_{2} \mathrm{O}$ w/ $0.1 \%$ formic acid (A) and $\mathrm{MeCN}(\mathrm{B})$.

\begin{tabular}{crl}
\hline$t(\min )$ & $\mathrm{A}$ & $\mathrm{B}$ \\
\hline 0.0 & 80 & 20 \\
10.0 & 5 & 95 \\
15.0 & 5 & 95 \\
20.0 & 80 & 20 \\
\hline
\end{tabular}

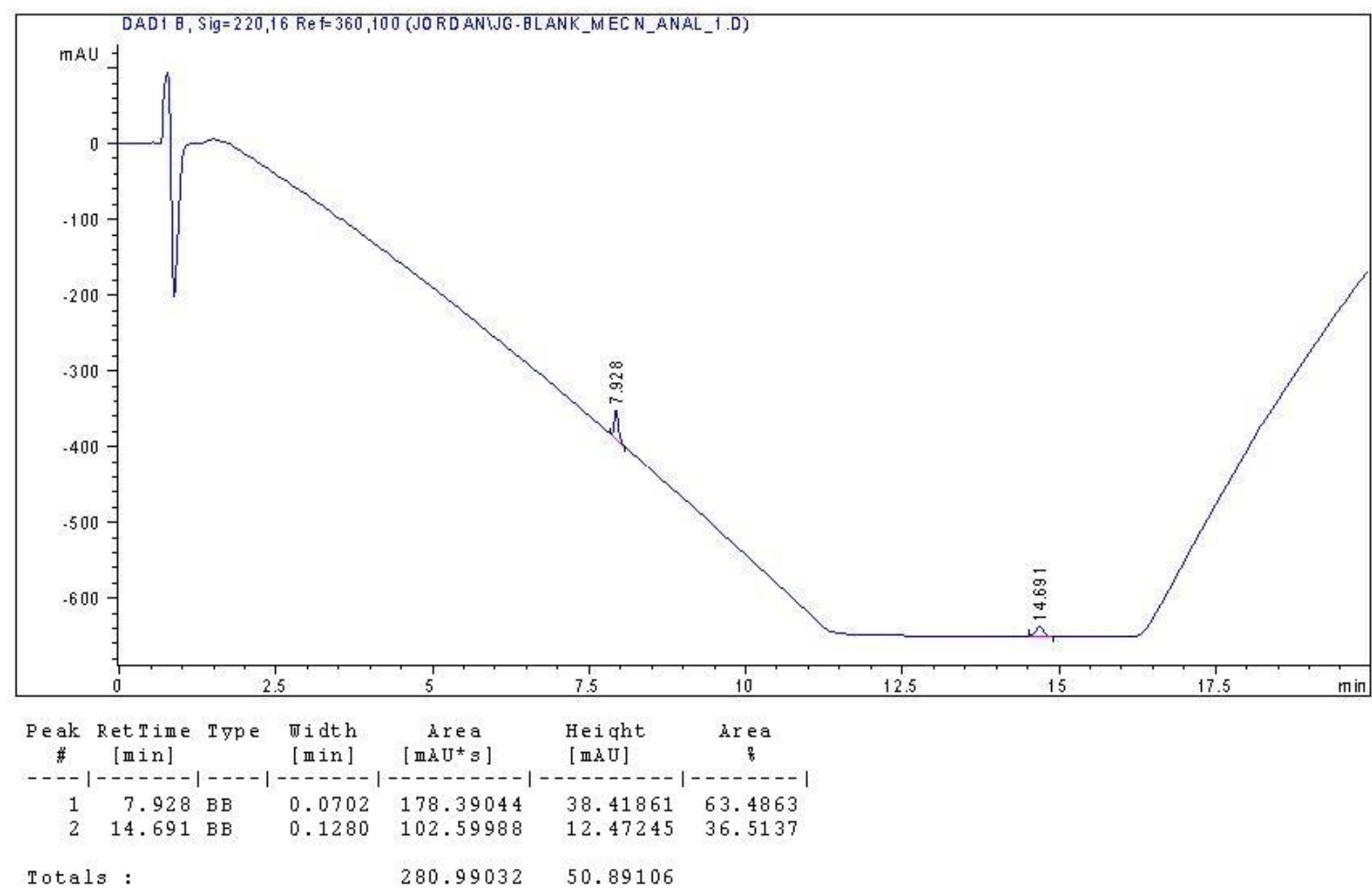

Figure S3. HPLC chromatogram of a blank injection ( $20 \mu \mathrm{L}$ of HPLC grade acetonitrile). 


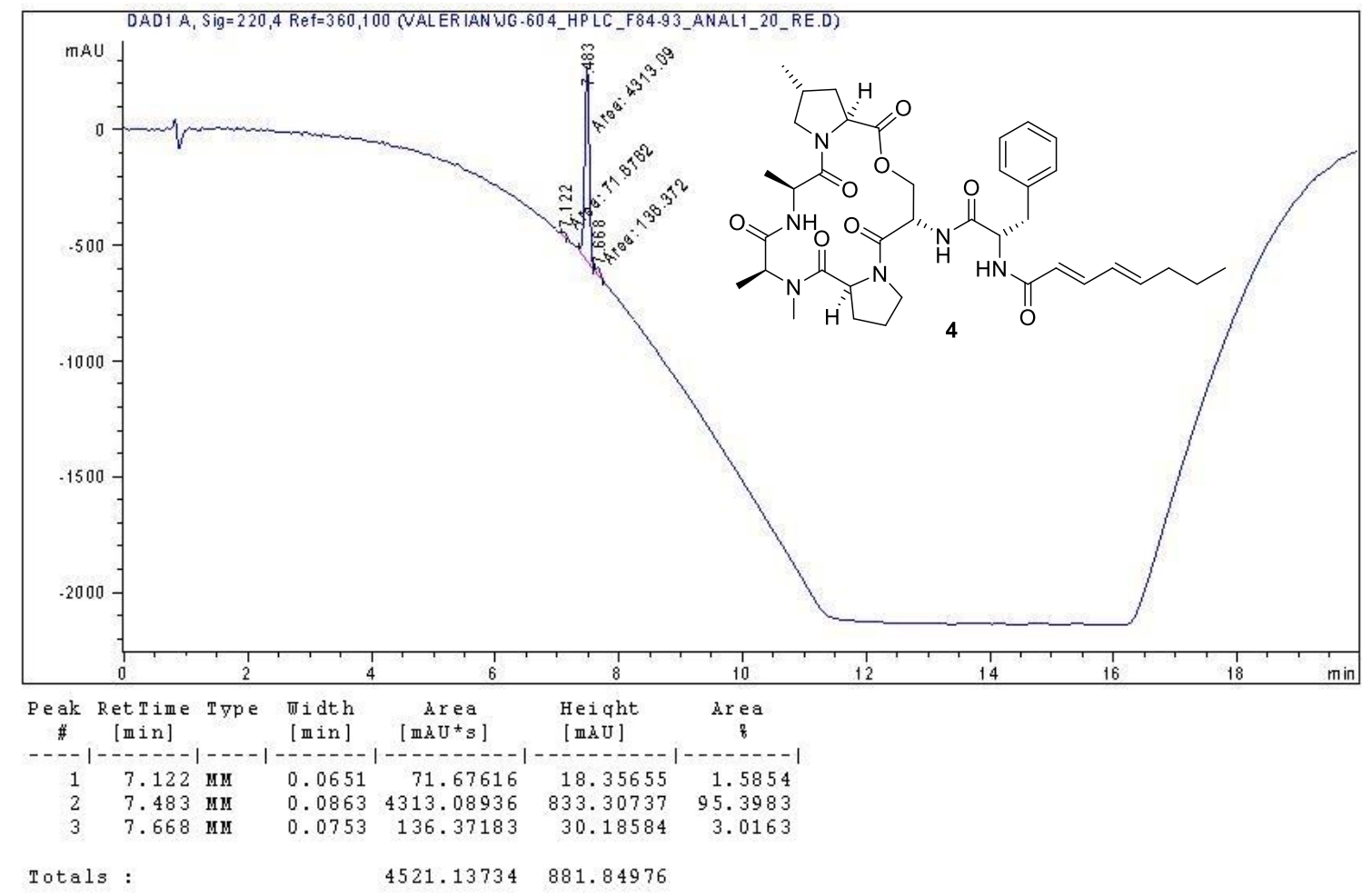

Figure S4. HPLC chromatogram of A54556 Factor D (4).

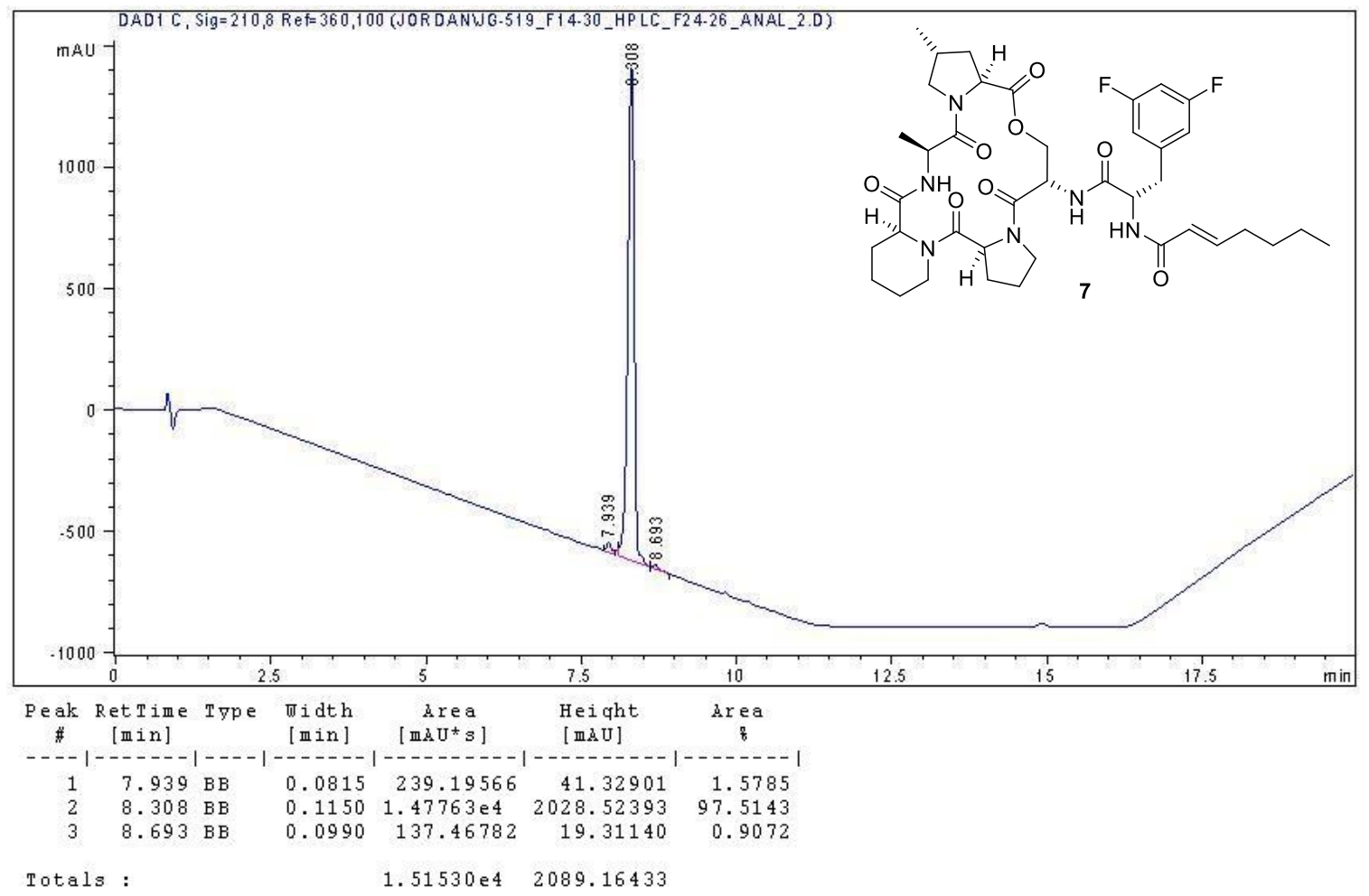

Figure S5. HPLC chromatogram of ADEP 4 (7). 


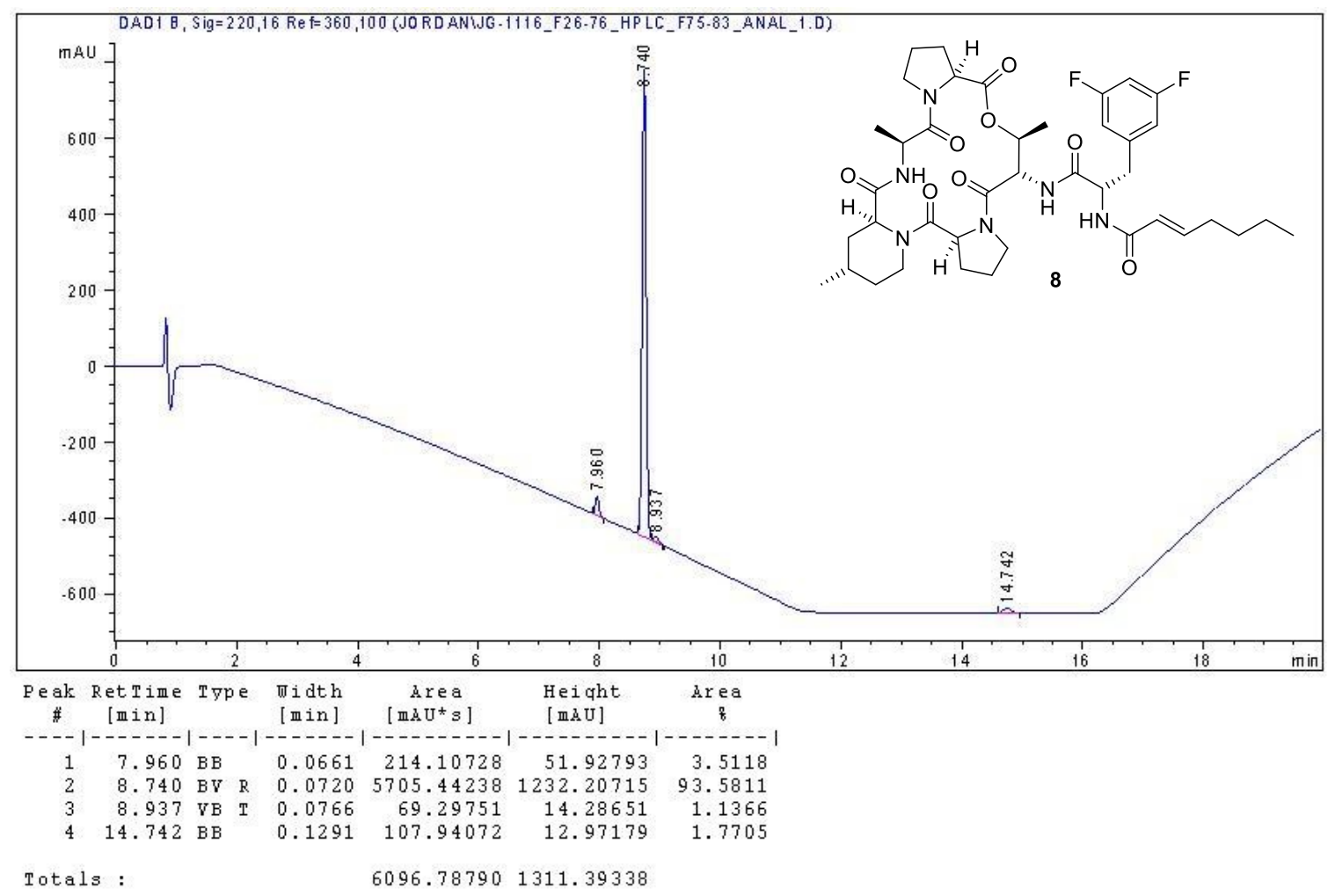

Figure S6. HPLC chromatogram of ADEP 8.

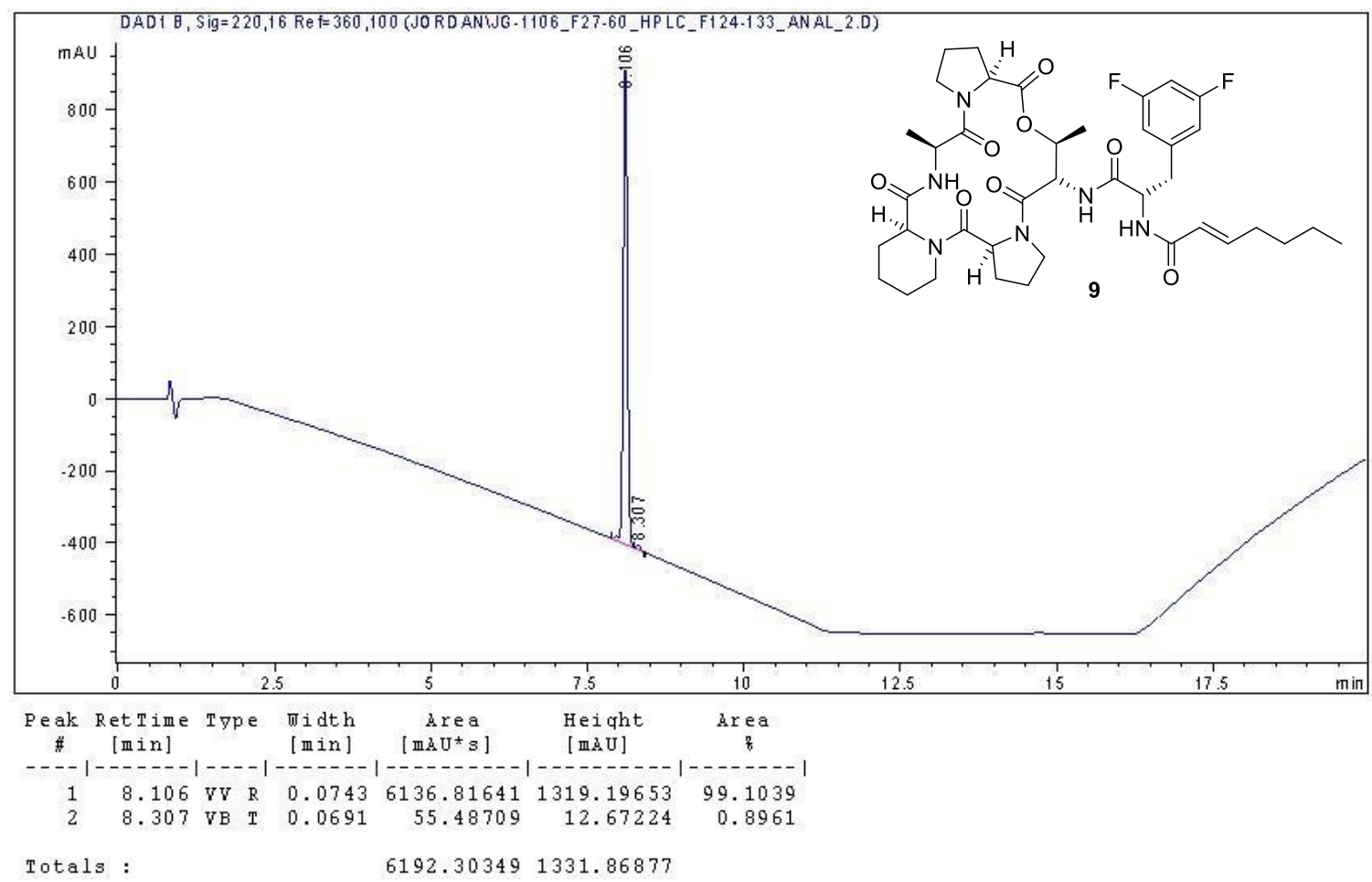

Figure S7. HPLC chromatogram of ADEP 9. 


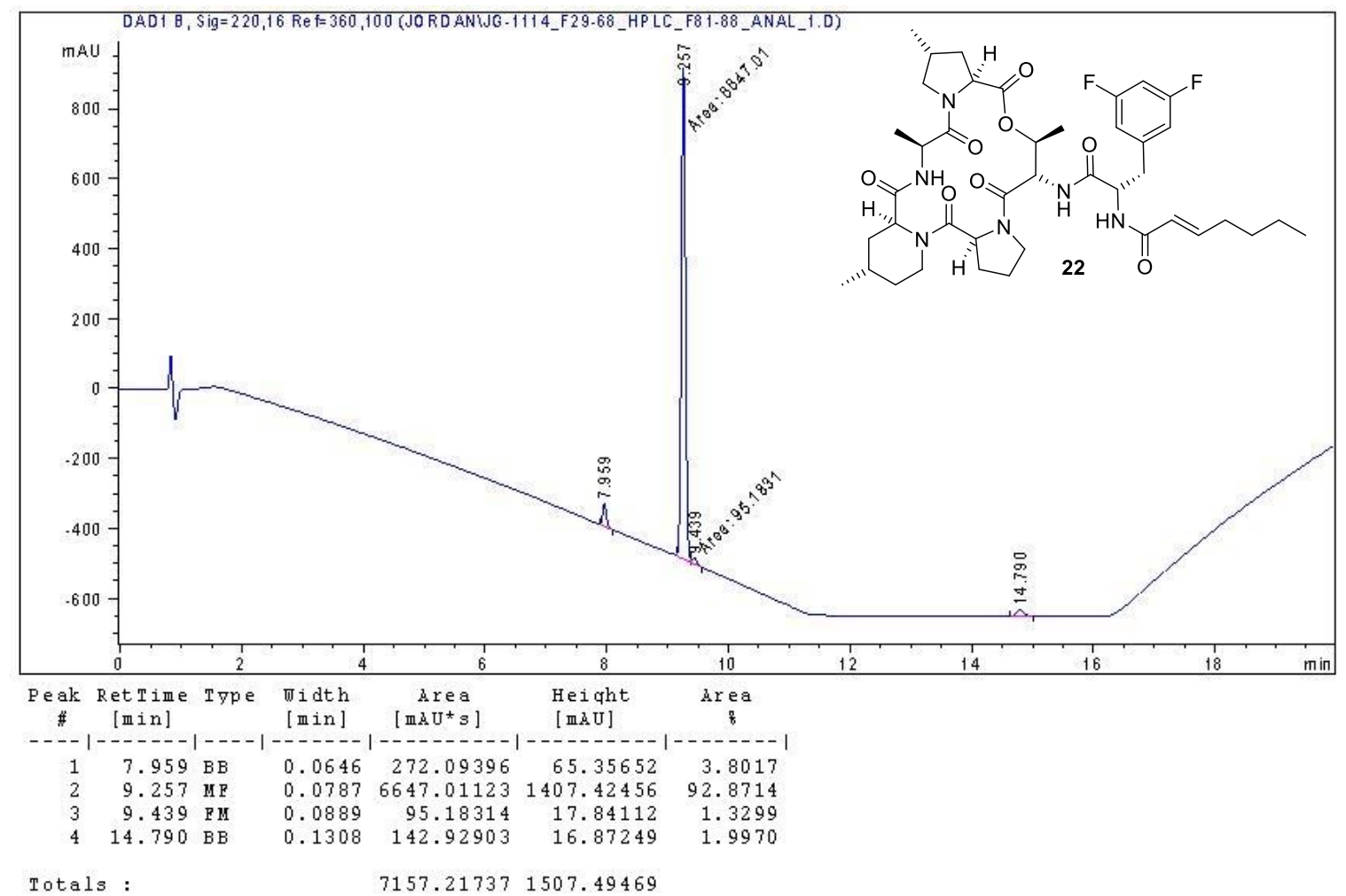

Figure S8. HPLC chromatogram of ADEP 22.

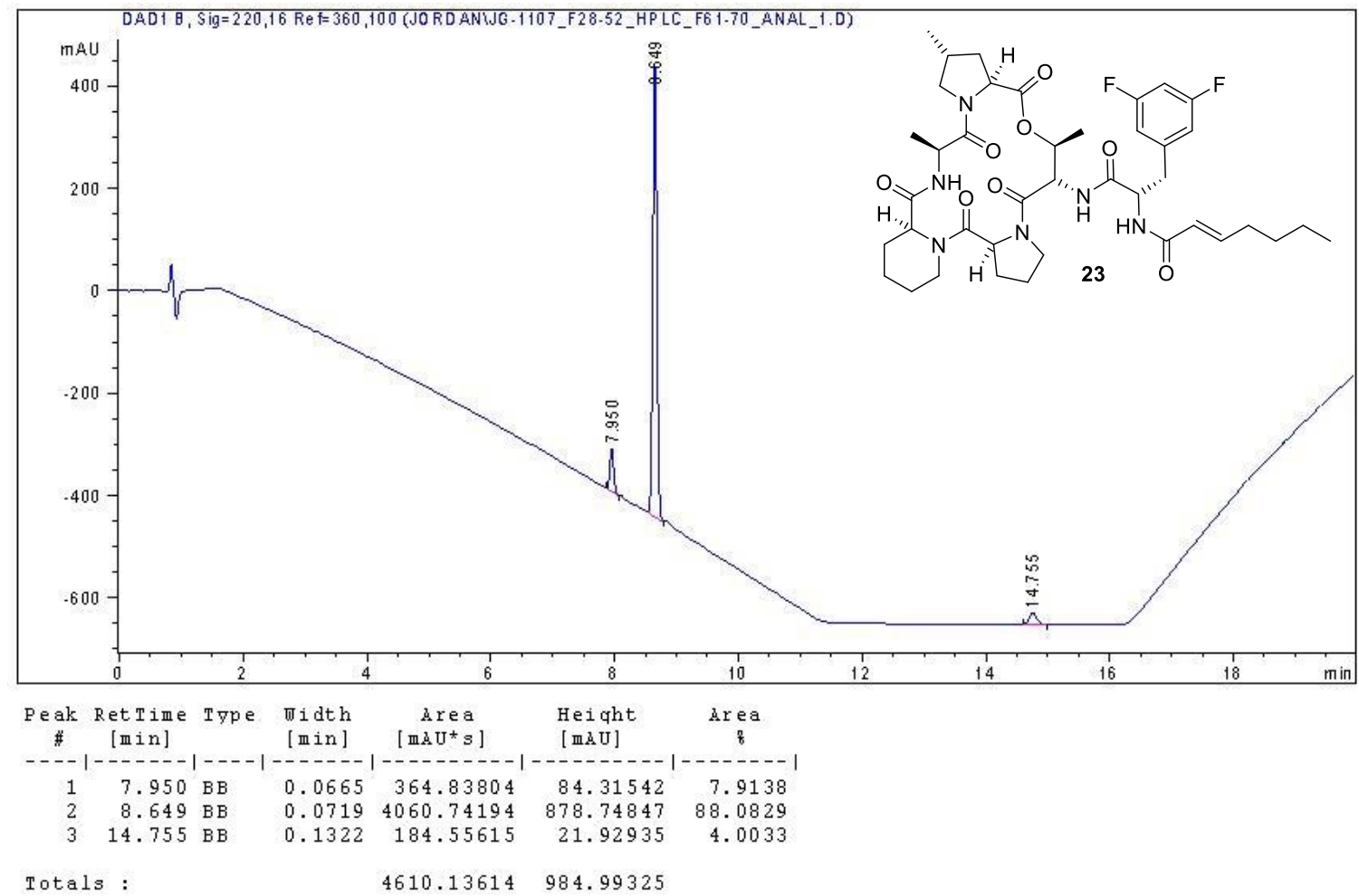

Figure S9. HPLC chromatogram of ADEP 23. 


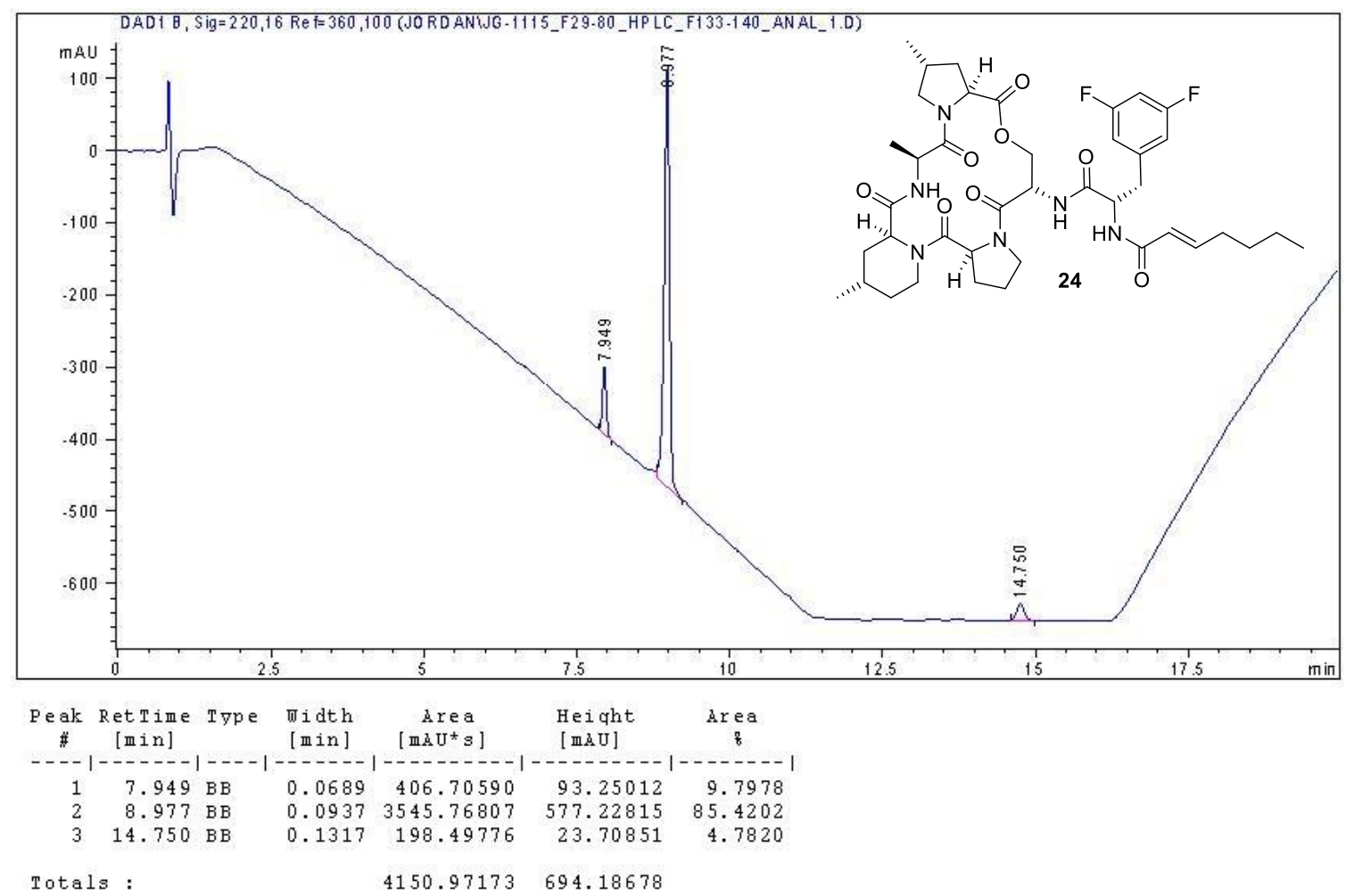

Figure S10. HPLC chromatogram of ADEP 24.

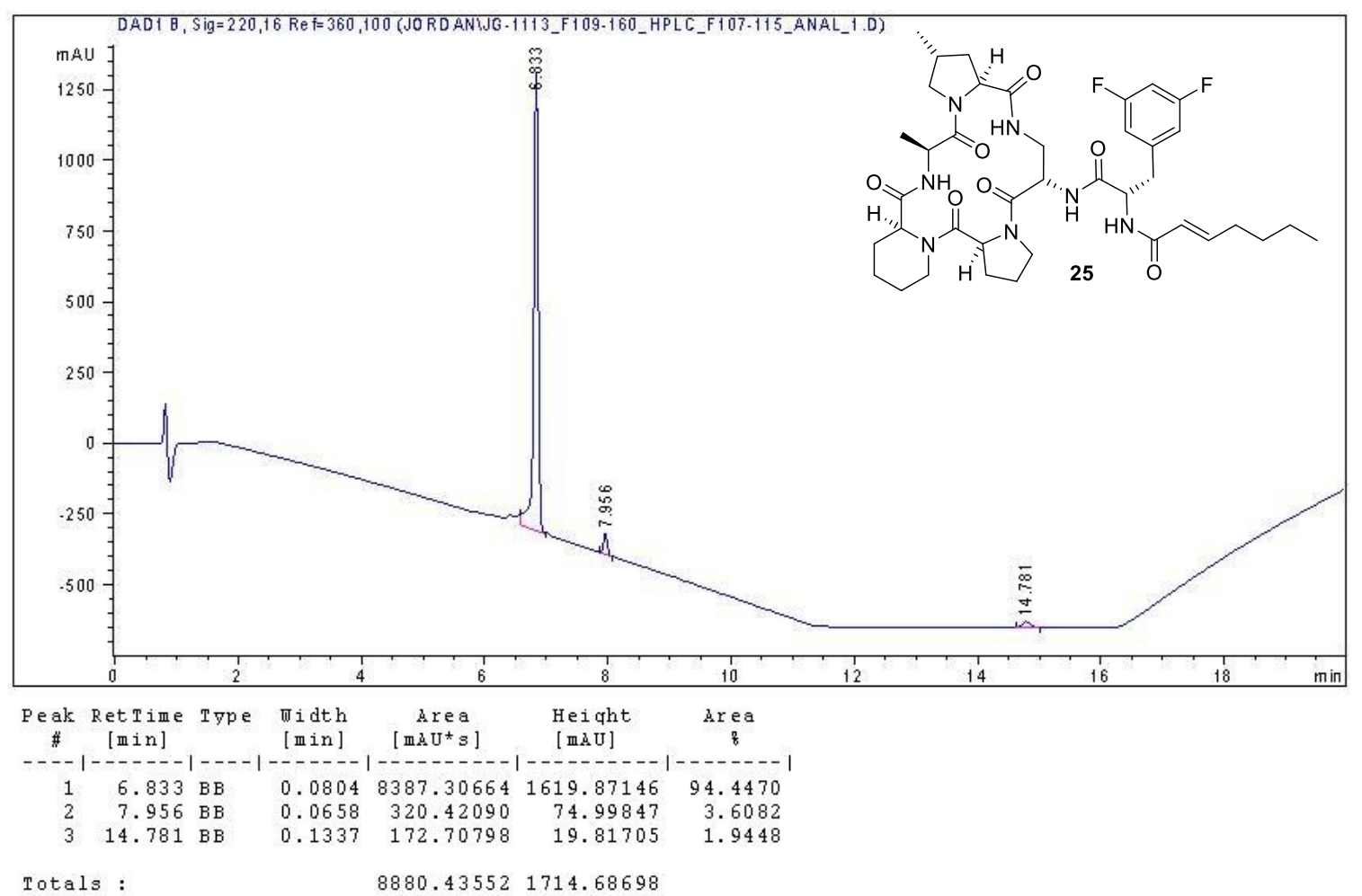

Figure S11. HPLC chromatogram of ADEP 25. 


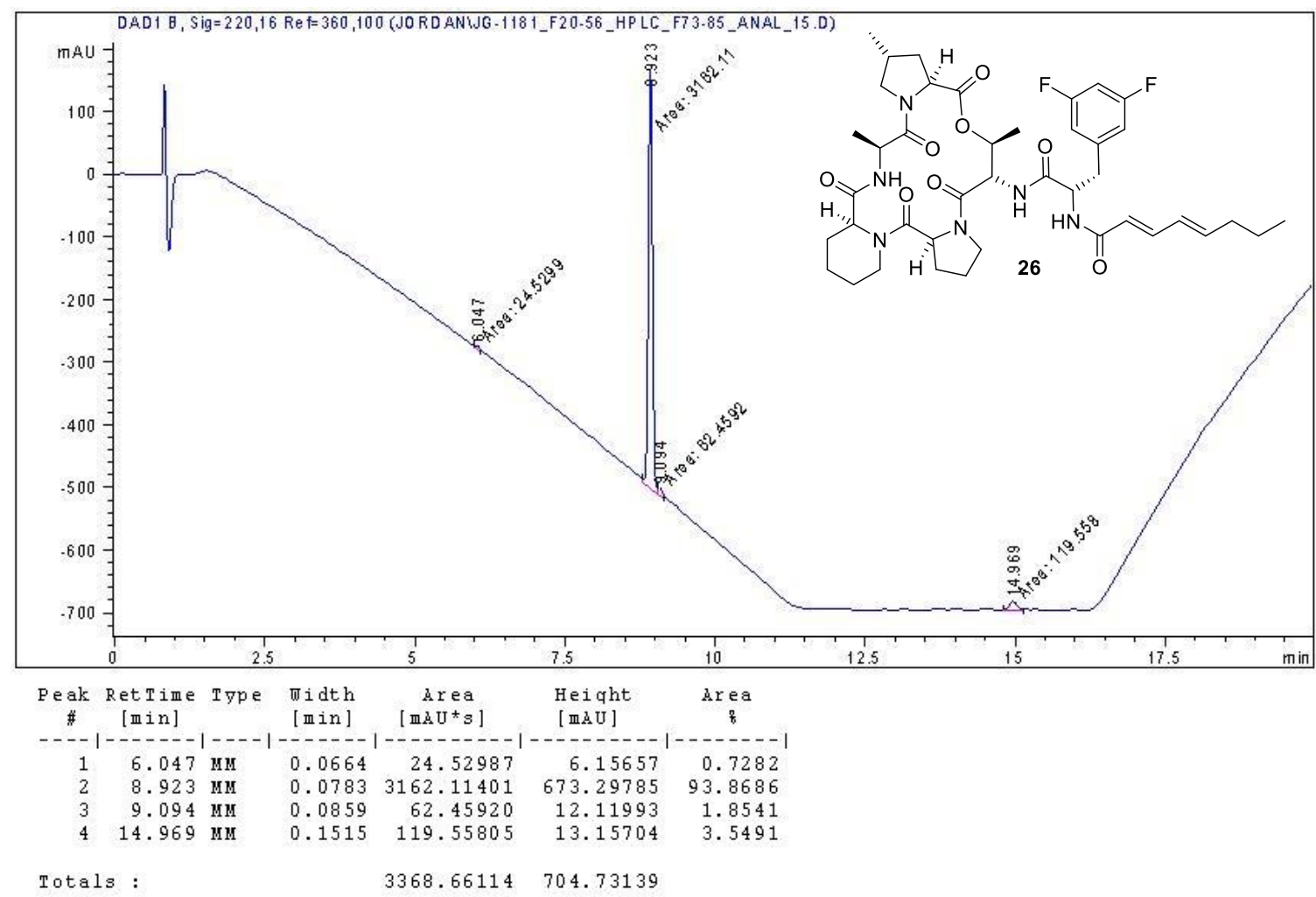

Figure S12. HPLC chromatogram of ADEP 26.

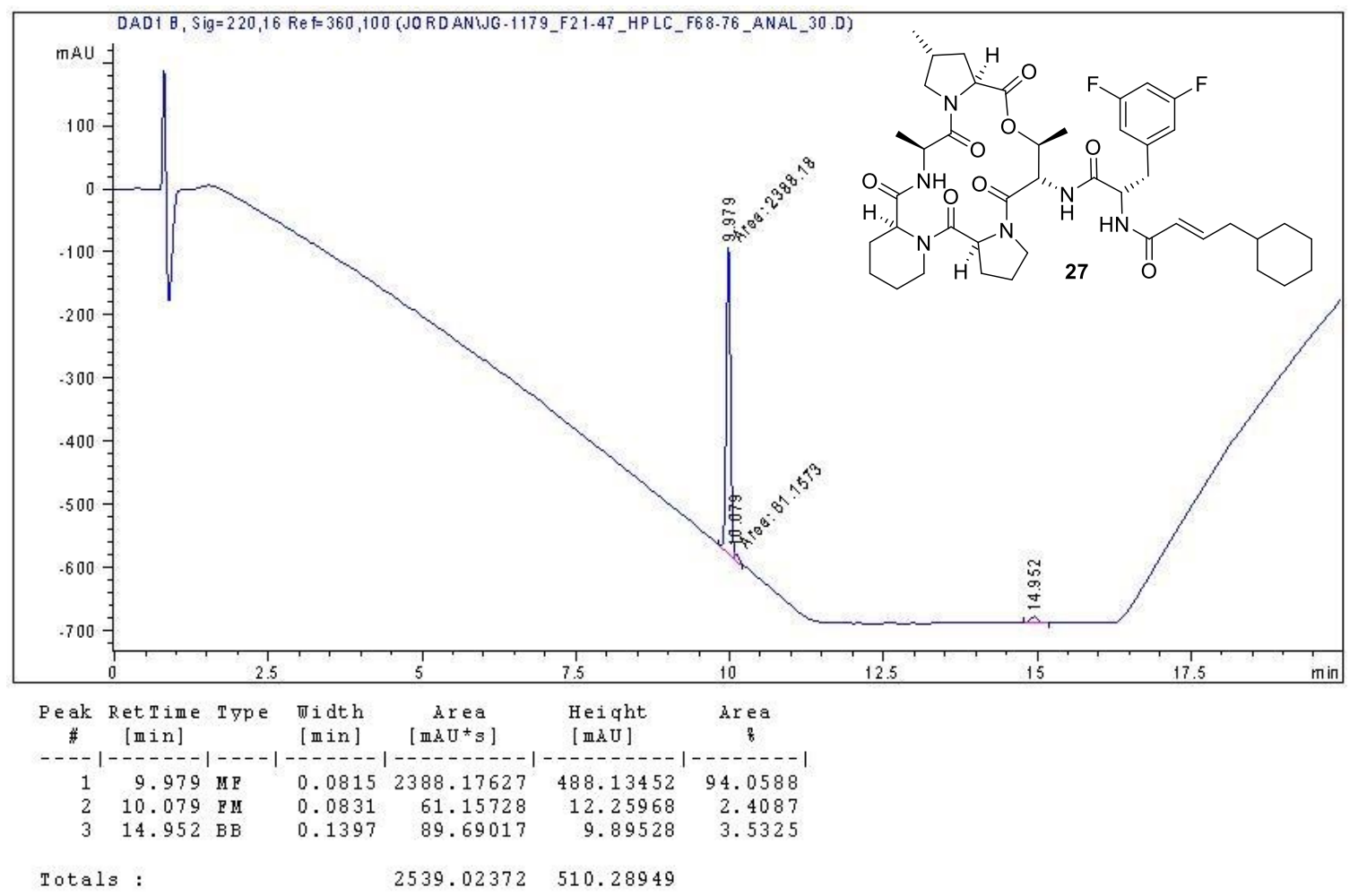

Figure S13. HPLC chromatogram of ADEP 27 


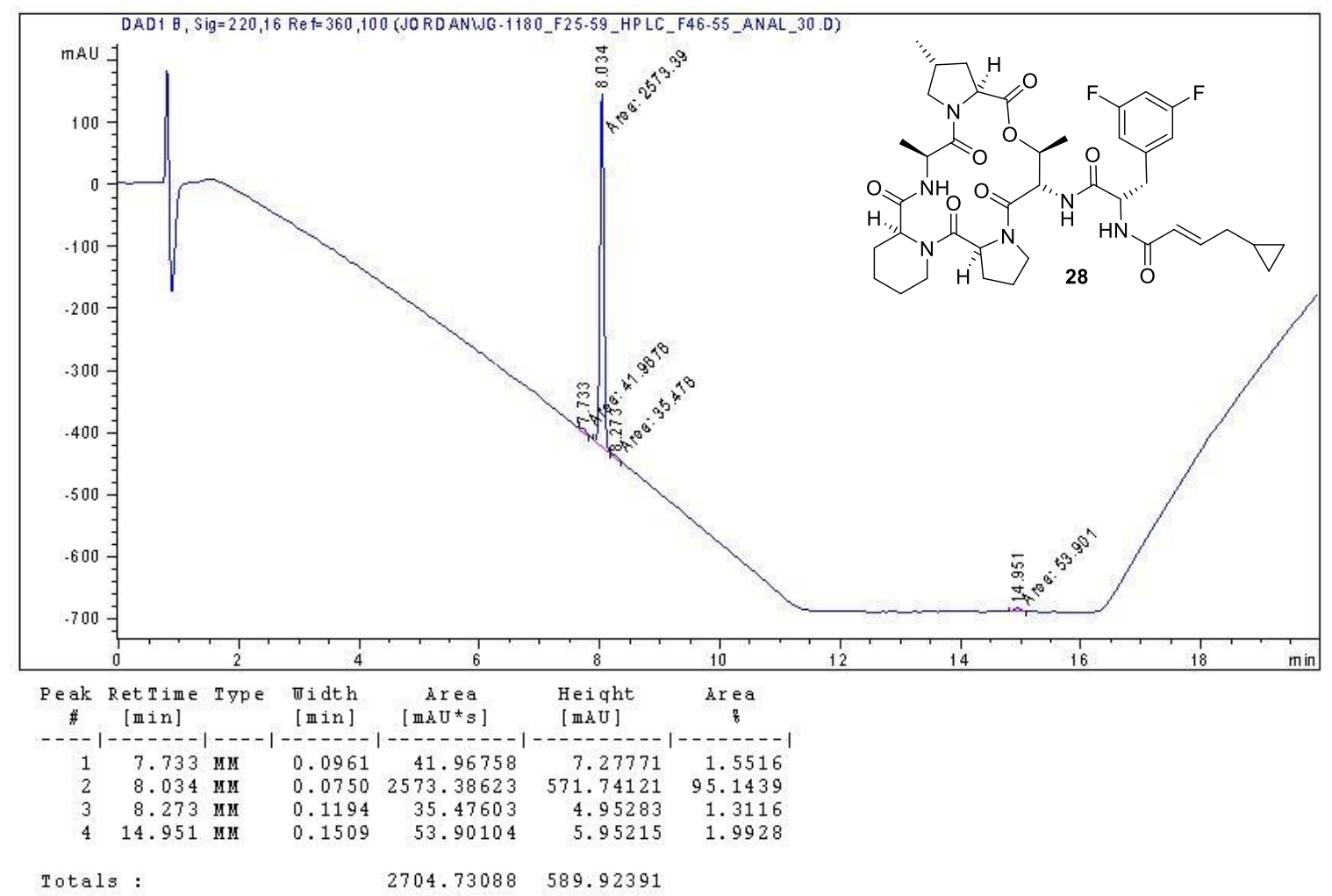

Figure S14. HPLC chromatogram of ADEP 28.

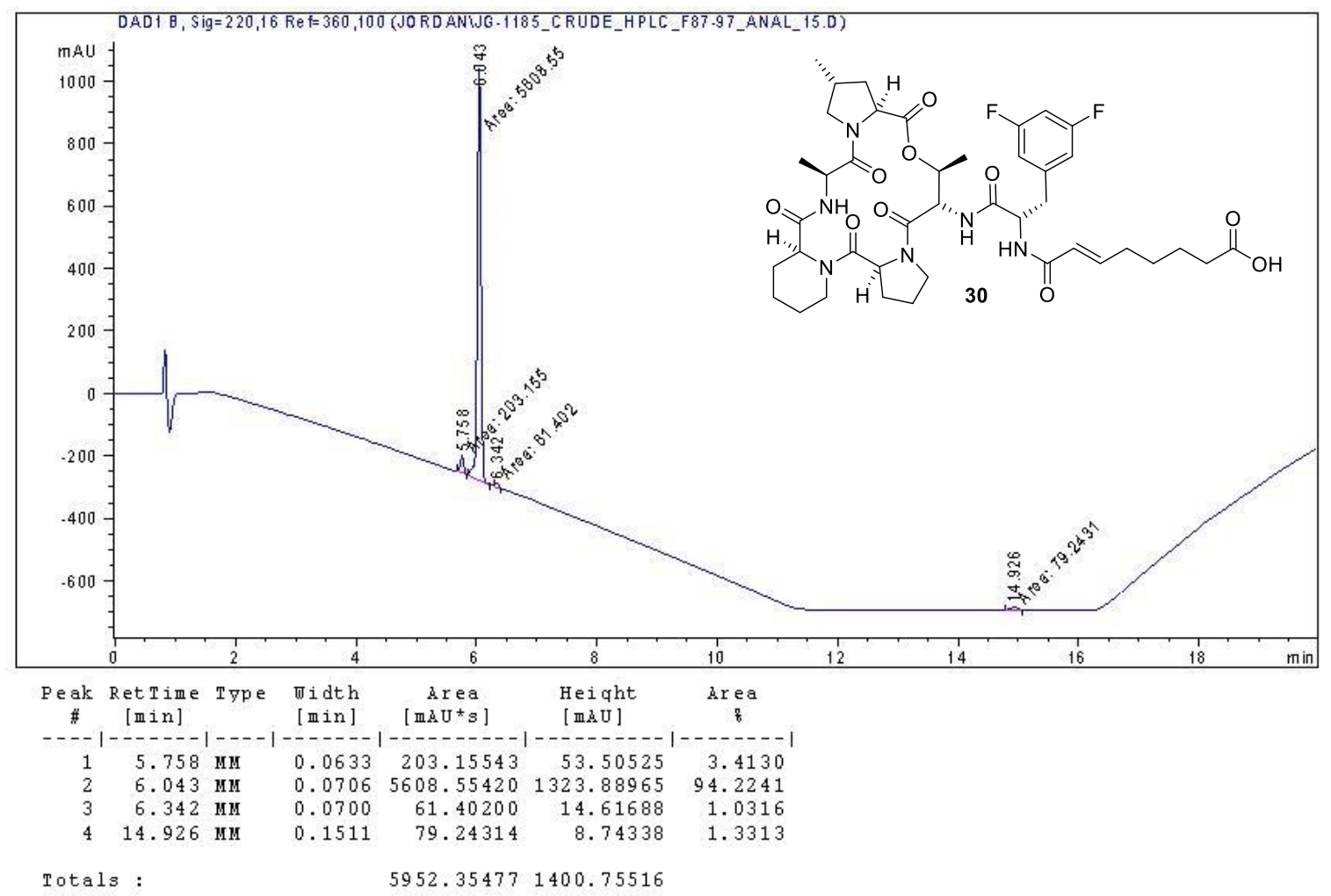

Figure S15. HPLC chromatogram of ADEP 30. 


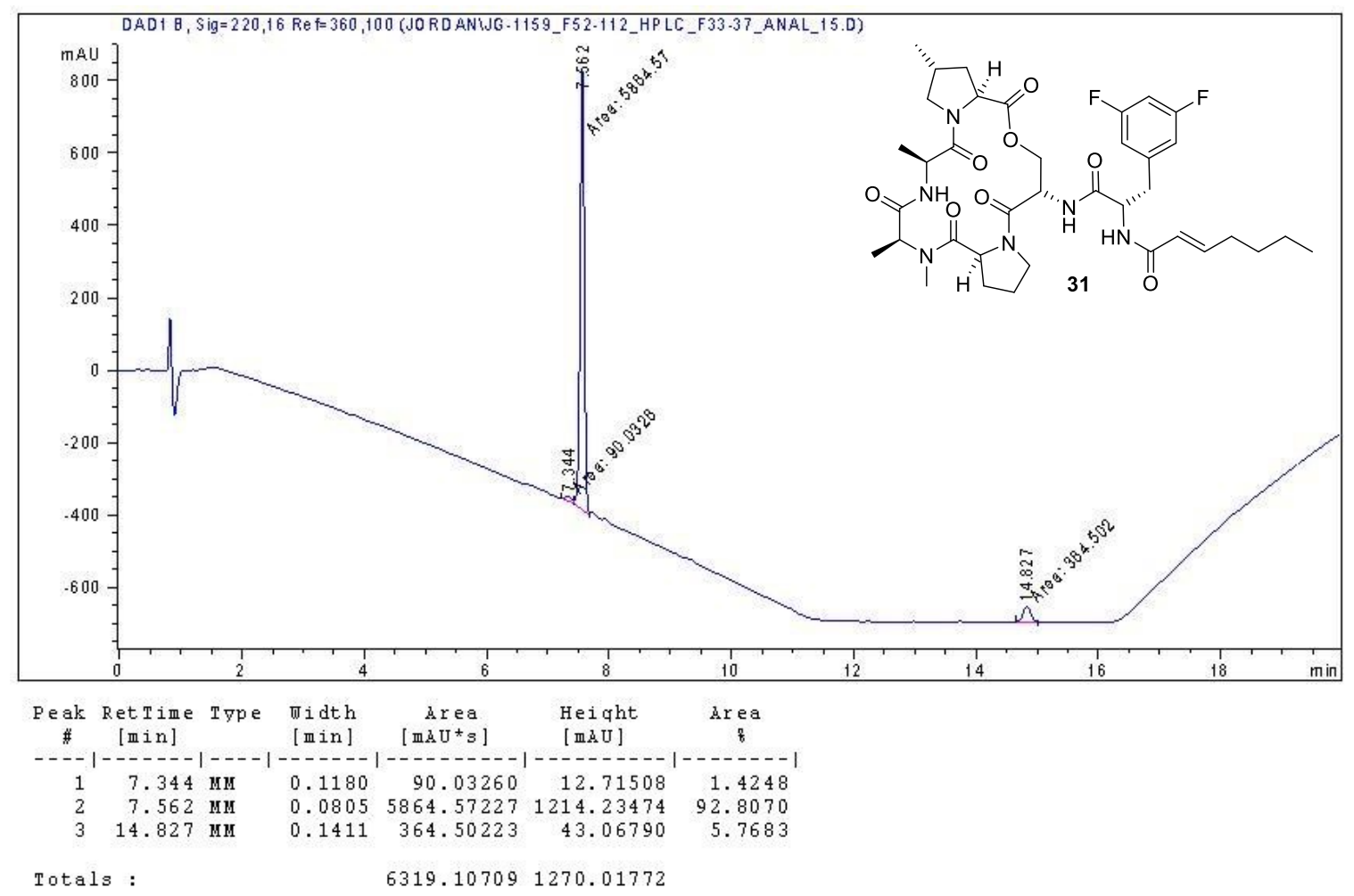

Figure S16. HPLC chromatogram of ADEP 31.

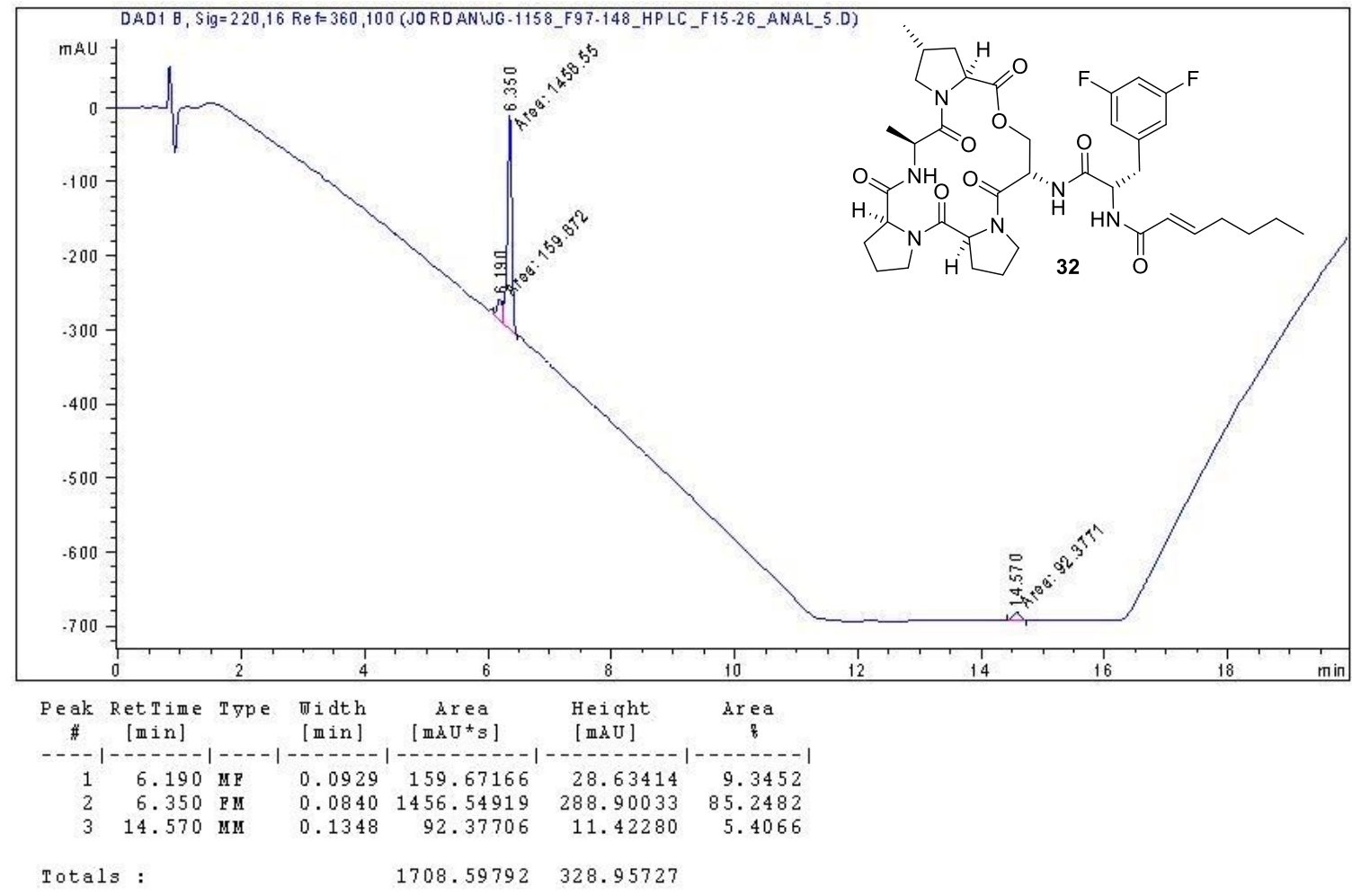

Figure S17. HPLC chromatogram of ADEP 32. 


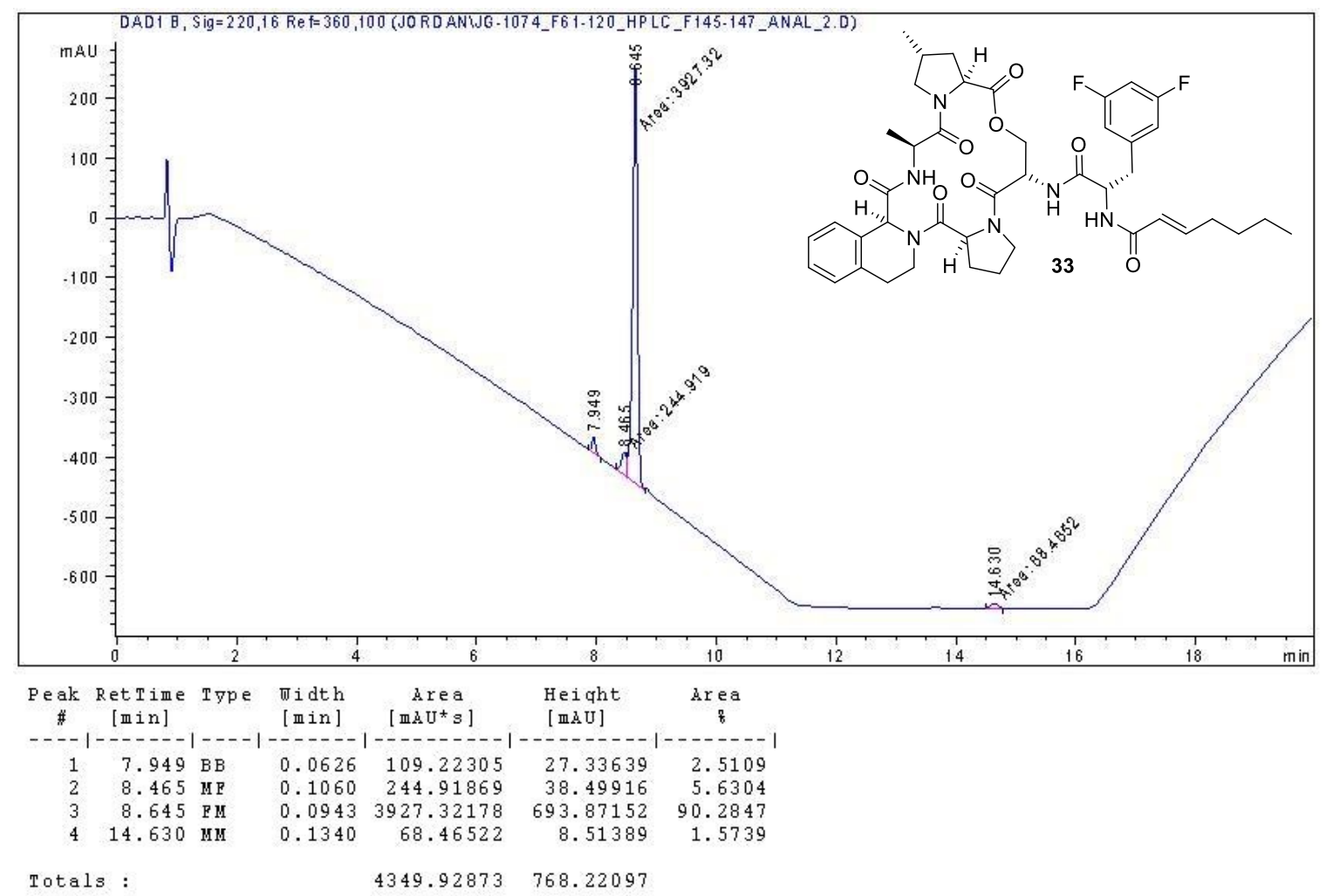

Figure S18. HPLC chromatogram of ADEP 33.

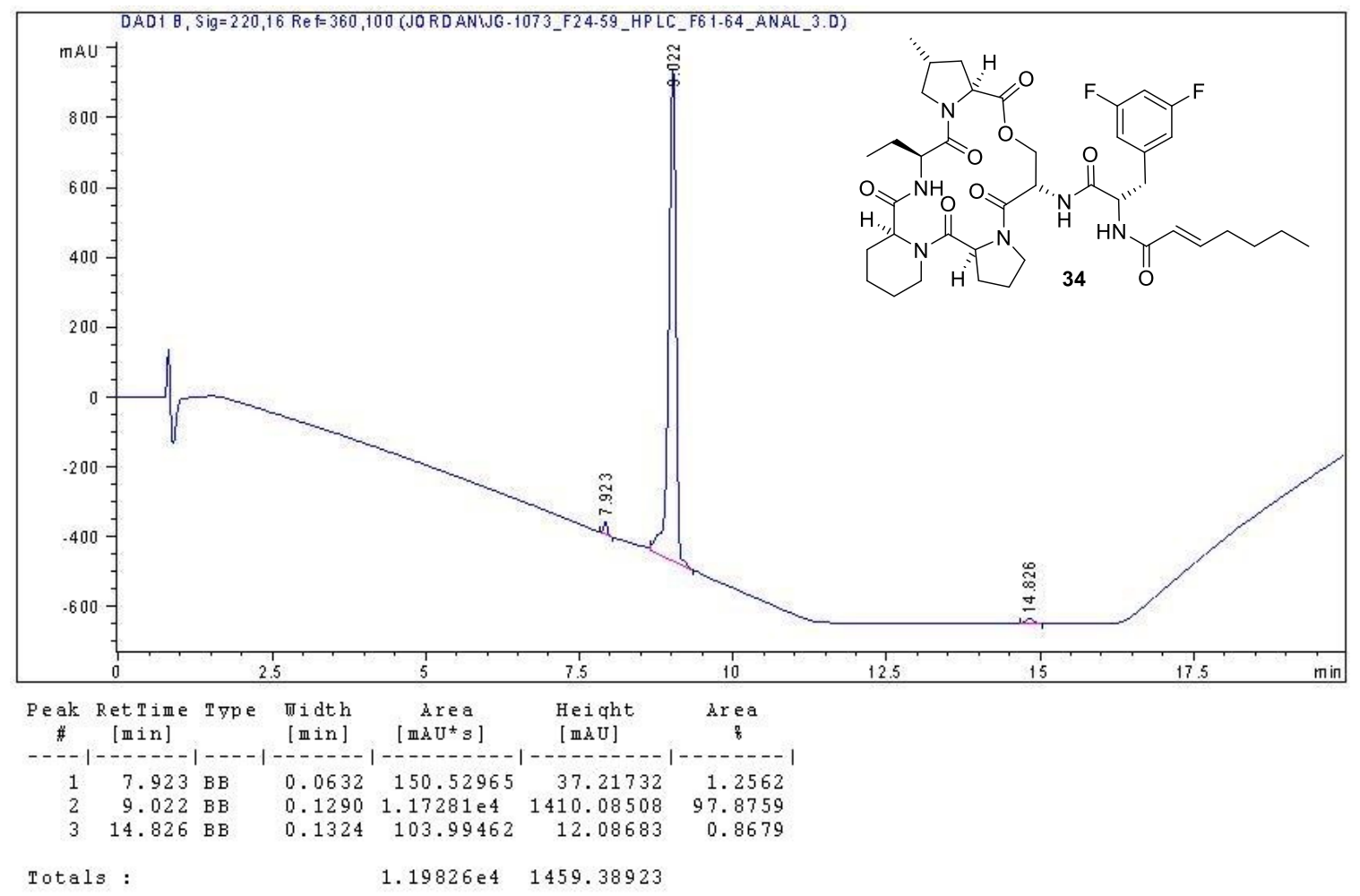

Figure S19. HPLC chromatogram of ADEP 34. 


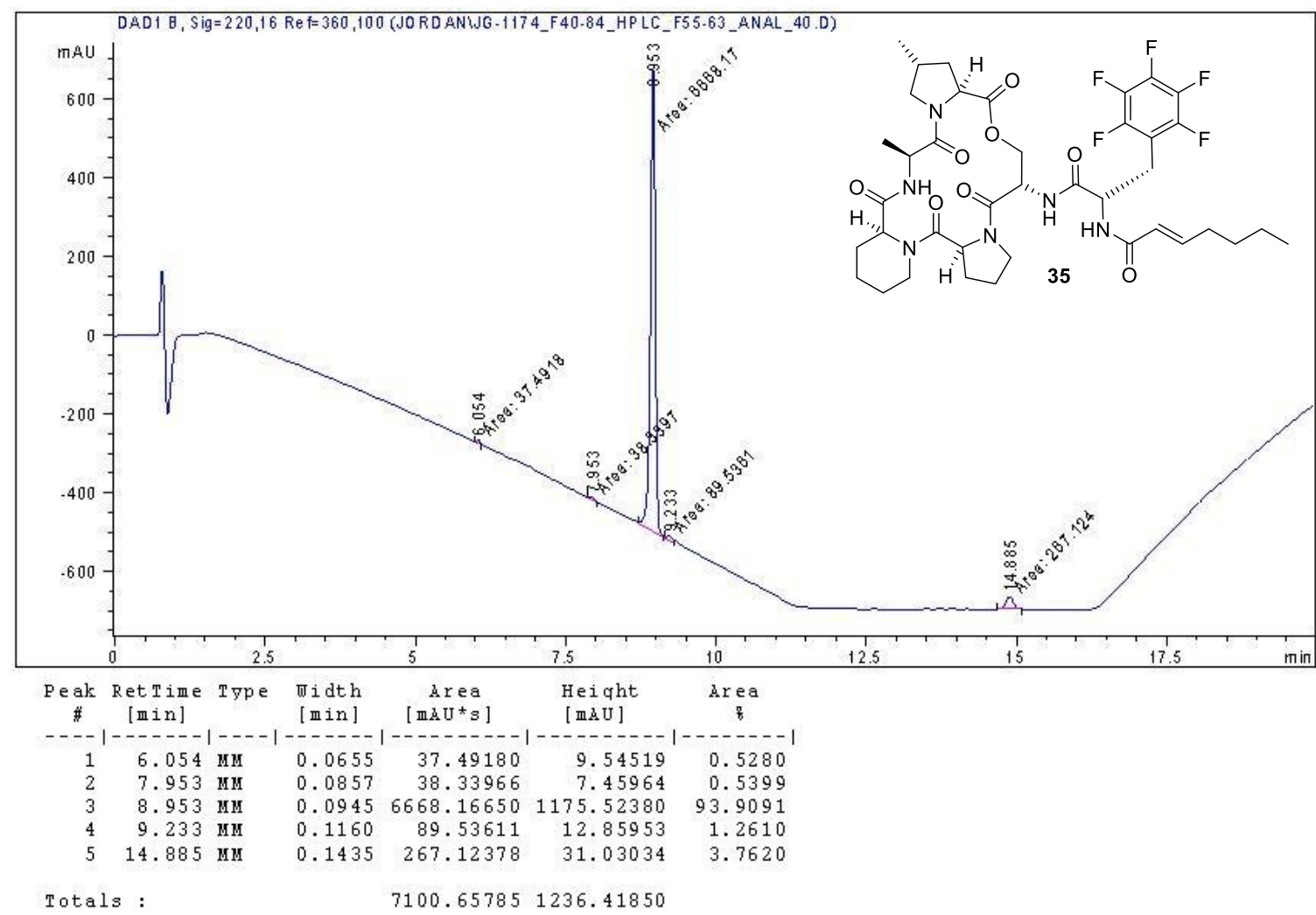

Figure S20. HPLC chromatogram of ADEP 35.

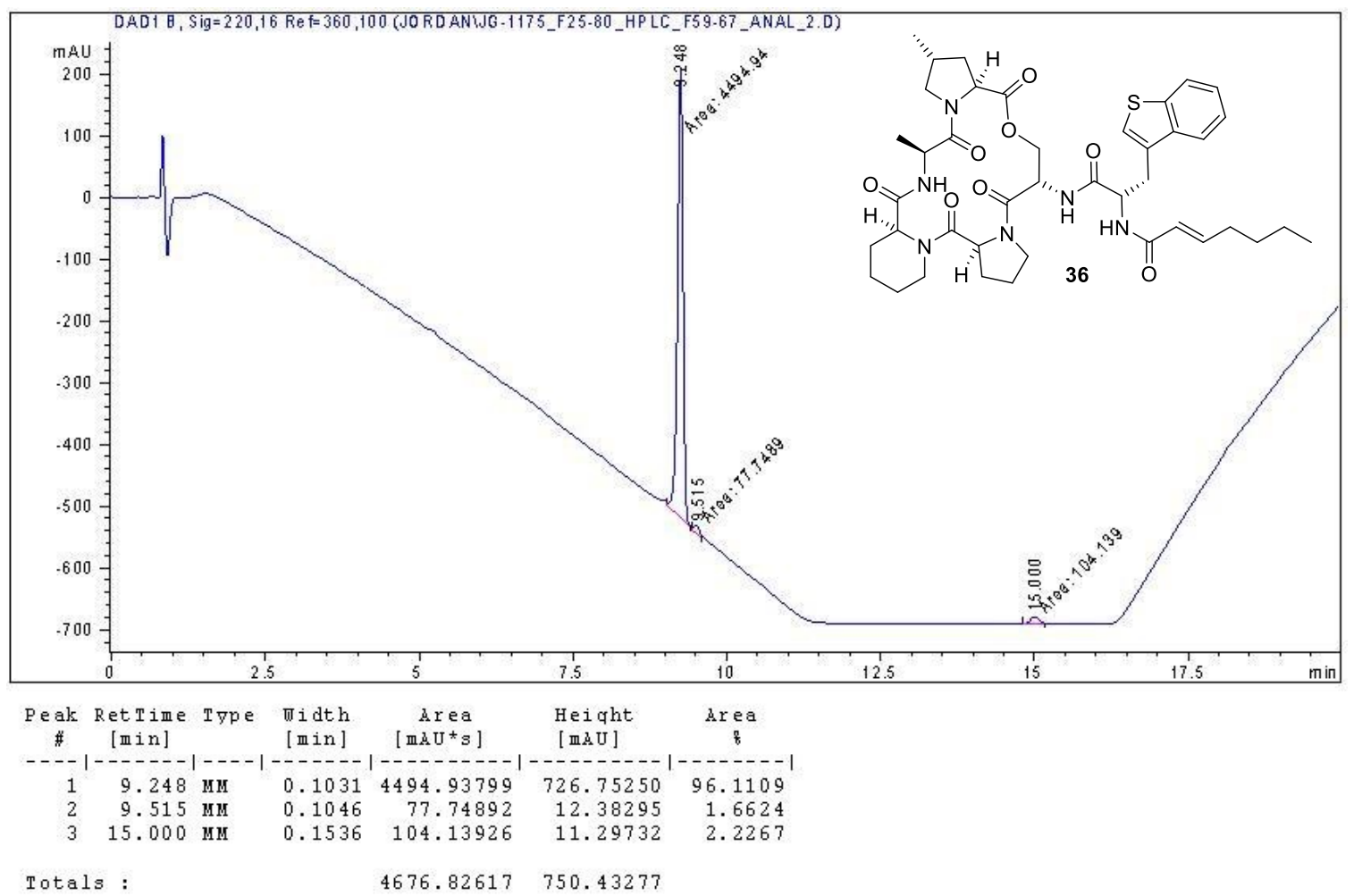

Figure S21. HPLC chromatogram of ADEP 36. 


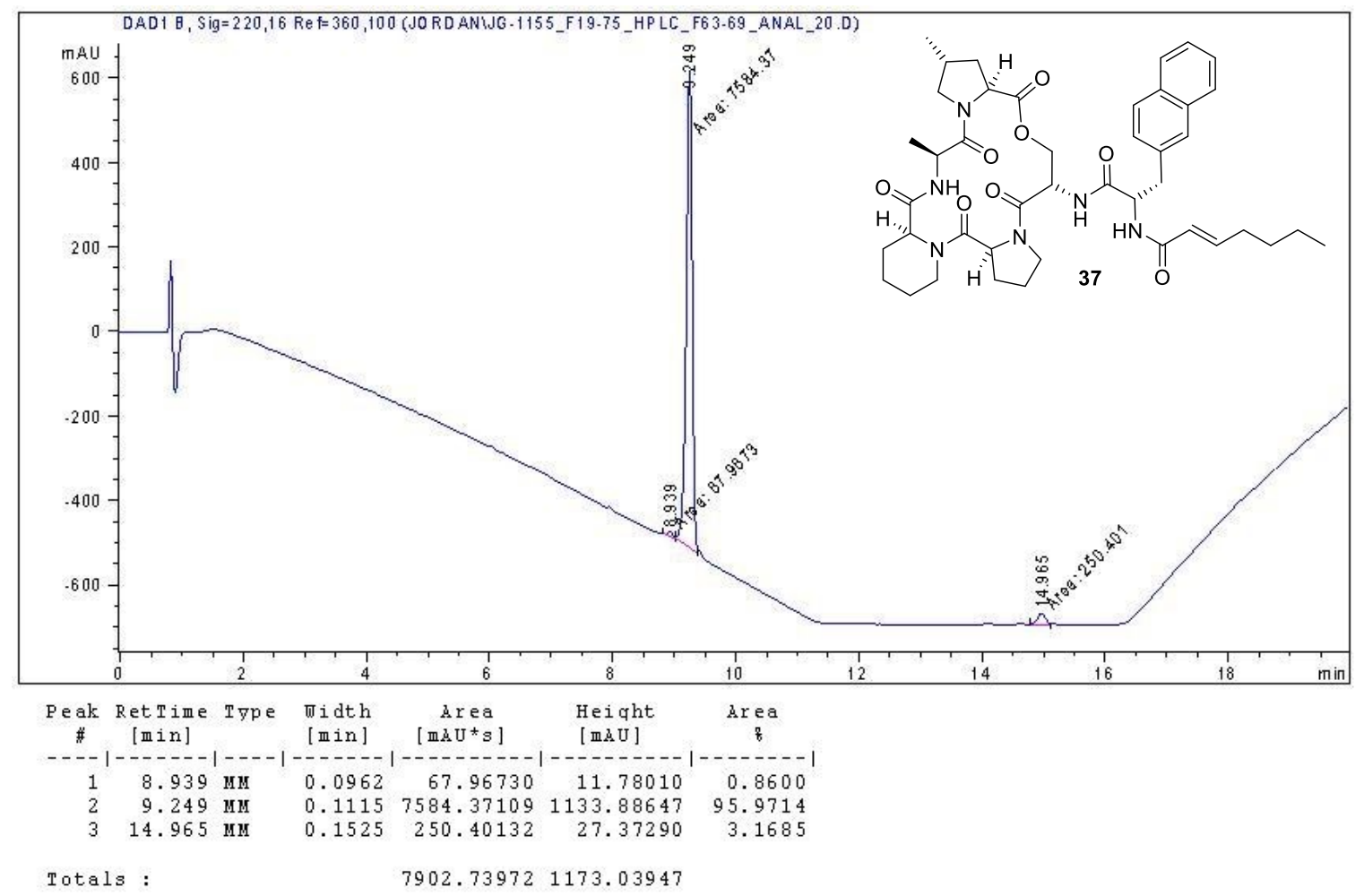

Figure S22. HPLC chromatogram of ADEP 37.

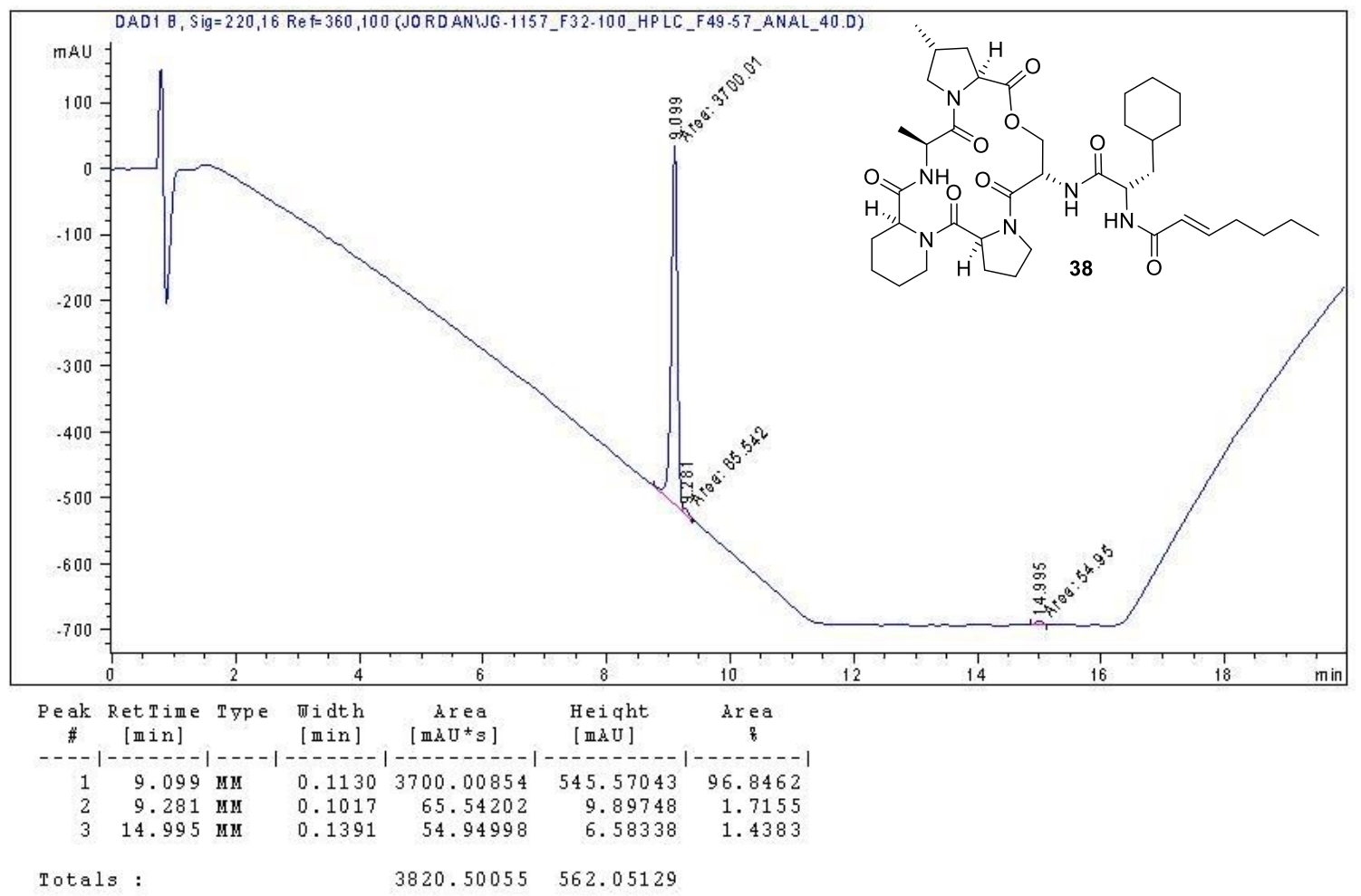

Figure S23. HPLC chromatogram of ADEP 38. 


\section{References}

(1) Sievers, F.; Wilm, A.; Dineen, D.; Gibson, T. J.; Karplus, K.; Li, W.; Lopez, R.; McWilliam, H.; Remmert, M.; Söding, J.; Thompson, J. D.; Higgins, D. G. Fast, scalable generation of high-quality protein multiple sequence alignments using Clustal Omega. Mol. Syst. Biol. 2011, 7, 539.

(2) Swoboda, J. G.; Meredith, T. C.; Campbell, J.; Brown, S.; Suzuki, T.; Bollenbach, T.; Malhowski, A. J.; Kishony, R.; Gilmore, M. S.; Walker, S. Discovery of a small molecule that blocks wall teichoic acid biosynthesis in Staphylococcus aureus. ACS Chem. Biol. 2009, 4, 875-883.

(3) Duthie E. S. Variation in the antigenic composition of staphylococcal coagulase. $J$. Gen. Microbiol. 1952, 7, 320-326.

(4) Strain obtained from ATCC: https://www.atcc.org/Products/All/29213.aspx

(5) Sahm, D. F.; Kissinger, J.; Gilmore, M. S.; Murray, P. R.; Mulder, R.; Solliday, J.; Clarke, B. In vitro susceptibility studies of vancomycin-resistant Enterococcus faecalis. Antimicrob. Agents Chemother. 1989, 33, 1588-1591.

(6) Youngman, P.; Perkins, J. B.; Losick, R. Construction of a cloning site near one end of Tn917 into which foreign DNA may be inserted without affecting transposition in Bacillus subtilis or expression of the transposon-borne erm gene. Plasmid 1984, 12, 1-9.

(7) Francis, K. P.; Yu, J.; Bellinger-Kawahara, C.; Joh, D.; Hawkinson, M. J.; Xiao, G.; Purchio, T. F.; Caparon, M. G.; Lipsitch, M.; Contag, P. R. Visualizing pneumococcal infections in the lungs of live mice using bioluminescent Streptococcus pneumoniae transformed with a novel Gram-positive lux transposon. Infec. Immun. 2001, 69, 3350-3358.

(8) Gründling, A.; Burrack, L. S.; Bouwer, H. G. A.; Higgins, D. E. Listeria monocytogenes regulates flagellar motility gene expression through MogR, a transcriptional repressor required for virulence. Proc. Nat. Sci. Acad. U. S. A. 2004, 101, $12318-12323$.

(9) Peters, J. E.; Thate, T. E.; Craig, N. L. Definition of the Escherichia coli MC4100 genome by use of a DNA array. J. Bacteriol. 2003, 185, 2017-2021.

(10) Sampson, B. A.; Misra, R.; Benson, S. A. Identification and characterization of a new gene of Escherichia coli K-12 involved in outer membrane permeability. Genetics 1989, 122, 491-501. 
(11) Stover, C. K.; Pham, X. Q.; Erwin, A. L.; Mizoguchi, S. D.; Warrener, P.; Hickey, M. J.; Brinkman, F. S. L.; Hufnagle, W. O.; Kowalik, D. J.; Lagrou, M.; Garber, R. L.; Goltry, L.; Tolentino, E.; Westbrock-Wadman, S.; Yuan, Y.; Brody, L. L.; Coulter, S. N.; Folger, K. R.; Kas, A.; Larbig, K.; Lim, R.; Smith, K.; Spencer, D.; Wong, G. K.-S.; Wu, Z.; Paulsen, I. T.; Reizer, J.; Saier, M. H.; Hancock, R. E. W.; Lory, S.; Olson, M. V. Complete genome sequence of Pseudomonas aeruginosa PAO1, an opportunistic pathogen. Nature 2000, 406, 959-964.

(12) Kupsch, E. M.; Knepper, B.; Kuroki, T.; Heuer, I.; Meyer, T. F. Variable opacity (Opa) outer membrane proteins account for the cell tropisms displayed by Neisseria gonorrhoeae for human leukocytes and epithelial cells. EMBO J. 1993, 12, 641-650.

(13) Piet, J. R.; Huis in 't Veld, R. A. G.; van Schaik, B. D. C.; van Kampen, A. H. C.; Baas, F.; van de Beek, D.; Pannekoek, Y.; van der Ende, A. Genome sequence of Neisseria meningitidis serogroup B strain H44/76. J. Bacteriol. 2011, 193, 2371-2372.

(14) Leung, E.; Datti, A.; Cossette, M.; Goodreid, J.; McCaw, S. E.; Mah, M.; Nakhamchik, A.; Ogata, K.; El Bakkouri, M.; Cheng, Y.-Q.; Wodak, S. J.; Eger, B. T.; Pai, E. F.; Liu, J.; Gray-Owen, S.; Batey, R. A.; Houry, W. A. Activators of cylindrical proteases as antimicrobials: identification and development of small molecule activators of ClpP protease. Chem. Biol. 2011, 18, 1167-1178. 